\title{
Production and Applications of Neutrons Using Particle Accelerators
}

\author{
David L. Chichester
}

November 2009

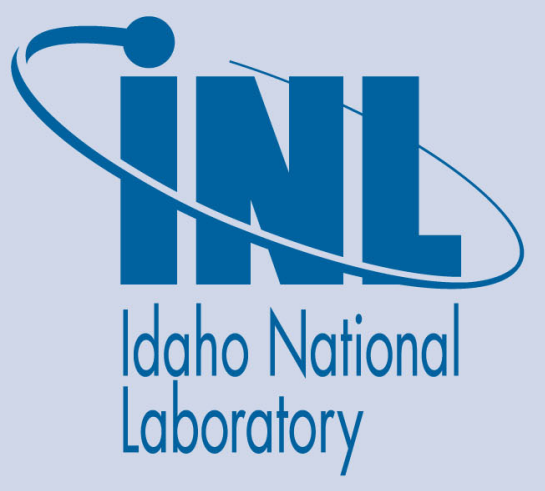

The INL is a U.S. Department of Energy National Laboratory operated by Battelle Energy Alliance 
INL/EXT-09-17312

\title{
Production and Applications of Neutrons Using Particle Accelerators
}

\author{
David L. Chichester
}

November 2009

\section{Idaho National Laboratory \\ Idaho Falls, Idaho 83415}

http://www.inl.gov

\author{
Prepared for the \\ U.S. Department of Energy \\ National Nuclear Security Administration \\ Under DOE Idaho Operations Office \\ Contract DE-AC07-05ID14517
}




\title{
PRODUCTION AND APPLICATIONS OF NEUTRONS USING PARTICLE ACCELERATORS
}

\author{
David L. Chichester \\ Idaho National Laboratory \\ 2525 North Fremont Avenue, Idaho Falls, Idaho, United States of America
}

\begin{abstract}
Advances in neutron science have gone hand in hand with the development and of particle accelerators from the beginning of both fields of study. Early accelerator systems were developed simply to produce neutrons, allowing scientists to study their properties and how neutrons interact in matter, but people quickly realized that more tangible uses existed too. Today the diversity of applications for industrial accelerator-based neutron sources is high and so to is the actual number of instruments in daily use is high, and they serve important roles in the fields where they're used. This chapter presents a technical introduction to the different ways particle accelerators are used to produce neutrons, an historical overview of the early development of neutron-producing particle accelerators, a description of some current industrial accelerator systems, narratives of the fields where neutron-producing particle accelerators are used today, and comments on future trends in the industrial uses of neutron producing particle accelerators.
\end{abstract}

\section{Introduction}

Following the particle acceleration and transmutation work of Cockcroft and Walton and the discovery of the neutron by Chadwick (both in 1932), it quickly followed that deuteron-deuteron disintegration reactions could be used to produce neutrons via the ${ }^{2} \mathrm{H}+{ }^{2} \mathrm{H} \rightarrow{ }^{3} \mathrm{H}+\mathrm{n}$ reaction in 1934.[1-5] Together these discoveries and other related work at Cambridge University's Cavendish Laboratory transformed the way experimental nuclear physics research was performed and, recognizing the usefulness of particle accelerators in nuclear physics experiments, more researchers soon followed along this path.[6-8] With the basic building blocks in place, it didn't take long for these early researchers 
to recognize the potential commercial utility of having electronic neutron sources for use in academia, medicine, industry, and beyond.[9-13]

Since that time particle-accelerator based neutron sources have been used in many different ways. Most noteworthy industrial applications have been on a small scale but one stands out above all others, namely the use of small, vacuumsealed electronic neutron generators (ENGs) for geophysical exploration in the petroleum industry. Beside this well known application examples of other interesting but less frequently seen commercial and industrial uses can be found for radiography, laboratory material characterization studies, on-line bulk material analyses, nuclear material assays, human and animal body-composition studies, and radiation effects testing. For applications requiring neutrons particle-accelerator based sources offer several key advantages over radioisotope neutrons sources such as the spontaneous fissioning isotope californium-252, or mixtures with alpha emitting radioisotopes that generate neutrons via the $(\alpha, n)$ reaction with low- $Z$ elements such as lithium or beryllium. Perhaps most important is that accelerator sources can be turned off, greatly simplifying issues related to safety, transportation, and storage of the source. Second, in most cases the accelerator based sources can also operate to produce pulses of neutrons. Pulsing opens up additional measurement approaches, including neutron dieaway time analyses, that steady-state sources cannot readily do. Also, pulsing allows for data to be collected during time gates, in between pulses in the absence of the external neutron source, which can be especially useful when measuring neutrons since much stronger signal to noise ratios may be achieved. Thirdly, several accelerator based neutron sources are monoenergetic, which again is often found to be a useful attribute in many applications. Also, for some accelerator based sources time of flight measurements are possible, either through the use of extremely short pulse widths are or through the use of associated particle time-tagging approaches.

Of course, there are several limitations associated with accelerator based neutron sources in comparison with radioisotope sources. For example, the operational reliability of radioisotopes is essentially perfect - they don't stop making neutrons. Accelerator based sources, in contrast, are complicated devices which are vulnerable to multiple failure modes from both external causes (e.g. loss of power, shock and vibration, extreme heat) and internal causes (e.g. component failures, vacuum leaks, ion beam target deterioration). Also, for source intensities less than about $\mathcal{O}\left(10^{9}\right)$ neutrons per second radioisotope sources are cheaper than accelerator based systems (for higher source strengths the storage, packaging, and handling challenges associated with radioisotopes sources begin to become significant). 


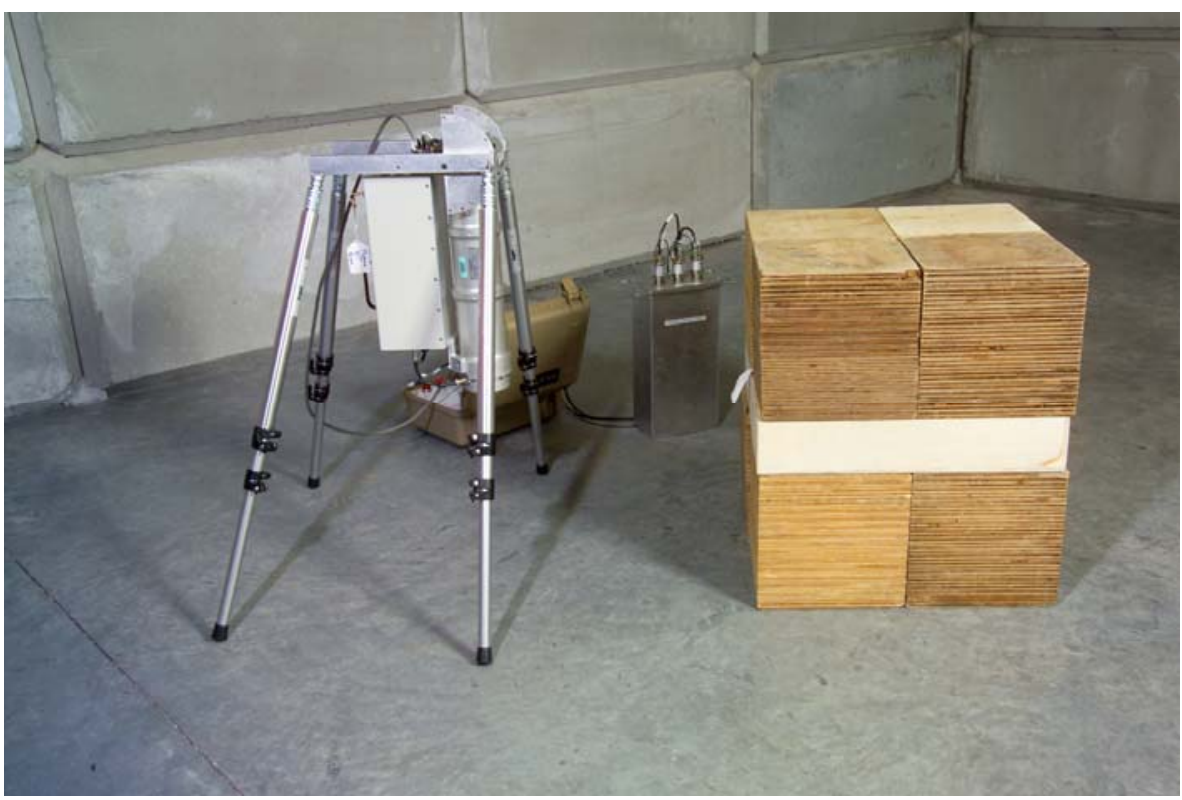

Fig. 1 Photograph of a typical compact electronic neutron generator, on the left, in a prototypical set-up reflecting how it can be used in active neutron interrogation. In this photo the interrogation object is a plywood cube measuring $60 \mathrm{~cm} \times 60 \mathrm{~cm} \times 60 \mathrm{~cm}$, to the right of picture. Directly behind the neutron generator is a beige battery control box capable of operating the neutron generator for $16+$ hours. To the right of the control box is a cadmium-shield, polyethylenemoderated ${ }^{3} \mathrm{He}$ detector. Not shown is the laptop computer needed to operate the system. The electronic neutron generator shown here measures approximately $15 \mathrm{~cm} \times 25 \mathrm{~cm} \times 60 \mathrm{~cm}$, weighs roughly $12 \mathrm{~kg}$, and produces $\mathcal{O}\left(10^{8}\right)$ neutrons per second.

Equally important is the fact that accelerator based sources are much larger than all radioisotope sources. This is immediately evident by examining the photograph of Fig. 1, showing a typical multi-purpose, portable electronic neutron generator $(\mathrm{ENG})$, and realizing that the size of a ${ }^{252} \mathrm{Cf}$ radioisotope neutron source needed to produce the same neutron intensity as this device is less than $2 \mathrm{~cm}^{3}$. Neutron source size is of particular importance in the petroleum industry where instrumentation must be designed to fit within narrow-diameter bore holes. It is important in other applications too, especially when neutron moderating materials, or more complicated assemblies designed to customize the emitted neutron energy spectrum, are needed and where the size and weight of these assemblies can grow large quickly depending upon the external dimensions of the accelerator that must be accommodated. Lastly, in most cases the cost of ownership for radioisotope sources based on either entry cost (cost per starting neutron emission rate) or total value of ownership (cost per emitted neutron) has 
for many years favored radioisotope sources. This has held for both short-lived sources such as ${ }^{252} \mathrm{Cf}$ as well as longer lived sources such as ${ }^{241} \mathrm{AmBe}$. Operational lifetimes for compact electronic neutron generators today stand at a few thousand hours of continuous use (usually with a decaying output overtime), before refurbishment and replacement parts are needed; larger, higher-yield systems typically require service on a more regular basis. In contrast, radioisotopes sources can operate with continuous, slowly decreasing outputs for years or decades before replacement is needed.

Despite the disadvantages listed above accelerator based neutron sources have found applications in many different niche areas. Looking towards the future the total use of these instruments is likely to continue to grow, both in absolute terms and in relative terms compared with radioisotope sources. General advances in the technology of radiation detection and measurement are coming at a steady and consistent rate, fueled primarily by evolutionary advances in basic physics, medicine, and the life sciences. New ideas and approaches for measuring radiation from these areas are spilling over into the industrial sector and these advances will inevitably serve to expand the overall breadth and depth of usage of neutron sources. Simultaneously time historical government subsidies associated with the production of radioisotopes are being reconsidered, leading to higher prices and reduced/less predictable availability, all making their use less economically favorable. At the same time commercial accelerator based solutions are continuously being improved and made more efficient and cost effective. Further, government and public acceptance of the use of commercial and industrial radioisotopes appears to be declining with perceived fears of poor security and the possibilities of their theft, misuse, and potential for diversion towards use in weapons of terror (radiological dispersal devices). Recent reports by the U.S. National Research Council and the North Atlantic Treaty Organization (NATO) underscore these concerns, research efforts are underway by many organizations to develop accelerator based replacements for all types of currently used radioisotopes.[14,15]

This aim of this chapter is to provide an overview of the technology of neutron-producing accelerators and to describe applications where they are used in industry. This starts with a section on the reactions accelerators use to produce neutrons, then descriptions of the equipment, and then an overview of examples where accelerator based neutron sources have been used in commerce and industry. While several different types of accelerators have been used for producing neutrons in industrial settings the sealed-vacuum accelerator systems, also called electronic neutron generators (ENGs), sealed-tube neutron generators (STNGs), or compact-accelerator neutron generators (CANGs), have found the 
most widespread application and are of particular emphasis here. Several excellent books have been written which provide detailed reviews of the underlying technology involved in ENGs including Fast Neutron Physics by Marion and Fowler, Activation Analysis with Neutron Generators by Nargolwalla and Przyblowicz, Neutron Sources for Basic Physics and Applications by Cierjacks (ed.), and the CRC Handbook of Fast Neutron Generators by Csikai.[16-19] Rather than duplicating this work this chapter will provide the basic knowledge needed for understanding accelerator-based neutron generators, their design, and operations; referring those interested in learning greater detail about these systems to these excellent reference sources. Large-scale research installations such as the Intense Pulsed Neutron Source (IPNS) at Argonne National Laboratory, the Los Alamos Neutron Science Center (LANSCE) at Los Alamos National Laboratory, and the Spallation Neutron Source (SNS) at Oak Ridge National Laboratory are not discussed in this chapter. While neutron science facilities like these certainly support industrial research their primary missions are for discovery and scientific exploration; their size and complexity mark them as unique one-of-a-kind instruments but they are far removed from the wider-scale commercial and industrial applications that are the subject of this text.

\section{Neutron Production}

The neutron is different from the other forms of radiation used in industrial applications in many ways. Most important is the fact that it is neutral in charge, making it insensitive to the electromagnetic interactions experienced by electrons, positrons, ions, and photons. Because of this, neutrons interact primarily with atomic nuclei rather than the electron clouds that surround them, making them ideal probes of the nuclear structure of matter and especially sensitive to different isotopes of the same element. In these interactions it is the kinetic energy (or speed) of the neutron that is of paramount importance in determining the probability and type of interaction that will take place with nuclei. At energies in the $\mathrm{MeV}$ range neutron inelastic scattering is the primary interaction while at thermal energies neutron capture is dominant.

One interesting fact about the neutron is that in a free state, unbound within an atom, it is unstable and undergoes radioactive and decay into a proton with a lifetime of 886 seconds, Eq. (1). Other physical parameters useful to know when working with neutrons are presented in Table 1 . A very important factor influencing how neutrons interact in matter is that the mass of a neutron is very 
similar to the mass of the proton, $1.00727646677 \mathrm{u}$, allowing elastic scattering with hydrogenous materials to transfer nearly all of a neutrons kinetic energy in a single scatter, quickly reducing a fast neutron's energy to thermal energies.

$$
n^{0} \rightarrow p^{+}+e^{-}+\bar{v}_{e}
$$

Table 1 Important parameters of the neutron.

\begin{tabular}{ccc}
\hline Parameter & Value & \\
\hline Neutron Mass & $1.674927211 \times 10^{-27}$ & {$[\mathrm{~kg}]$} \\
& 939.565346 & {$\left[\mathrm{MeV} / \mathrm{c}^{2}\right]$} \\
& 1.00866491597 & {$[\mathrm{u}]^{*}$} \\
Free Particle & $1.295 \times 10^{-3}$ & {$\left[\mathrm{~s}^{-1}\right]$} \\
Decay Constant & & \\
Free Particle & 535.2 & {$[\mathrm{~s}]$} \\
Half-life & & {$[\mathrm{s}]$} \\
Free Particle & 885.7 & \\
Mean Lifetime & & {$[\mathrm{m} / \mathrm{s}]$} \\
Neutron Velocity & & {$[\mathrm{cm} / \mathrm{ns}]$} \\
at $0.025 \mathrm{eV}$ & 2,200 & {$[\mathrm{~cm} / \mathrm{ns}]$} \\
at $2.5 \mathrm{MeV}$ & 2.2 & \\
at $14 \mathrm{MeV}$ & 5.2 &
\end{tabular}

${ }^{*}$ Atomic mass unit, equal to $1 / 12$ the mass of a ${ }^{12} \mathrm{C}$ atom.

\subsection{Reactions With Ions}

Fusion of light nuclei is the most common approach used with particle accelerators to generate neutrons; the most common of these reactions used for producing neutron are listed in Table 2. While other reactions also exist and have been used with accelerators these are the ones that have been found to be the most useful, the easiest to employ, and the most efficient for the purpose. All of these reactions have been used in open-vacuum accelerator systems but modern sealed-vacuum ENGs only use the ${ }^{2} \mathrm{H}$ (deuterium, D) and ${ }^{3} \mathrm{H}$ (tritium, T) fusion reactions. Further, due to the radiation safety complications associated with using tritium, its use is generally limited in current-generation industrial accelerators to only sealed-vacuum systems.[20,21] 
Table 2 Common nuclear reactions particle accelerators use to produce neutrons.

\begin{tabular}{|c|c|c|c|c|c|}
\hline \multirow{2}{*}{$\frac{\text { Reaction }}{{ }^{2} \mathrm{H}+{ }^{2} \mathrm{H} \rightarrow{ }^{3} \mathrm{He}+\mathrm{n}}$} & \multirow{2}{*}{$\begin{array}{l}\text { Shorthand } \\
{ }^{2} \mathrm{H}(\mathrm{d}, \mathrm{n})^{3} \mathrm{He}\end{array}$} & \multirow{2}{*}{$\begin{array}{c}\text { Q Value } \\
{[\mathrm{MeV}]}\end{array}$} & \multirow{2}{*}{$\begin{array}{c}\begin{array}{c}\text { Threshold } \\
\text { Energy } \\
{[\mathrm{MeV}]}\end{array} \\
\text { NA }\end{array}$} & \multicolumn{2}{|c|}{$\begin{array}{c}\text { Minimum Product } \\
\text { Energies } \\
{[\mathrm{MeV}]}\end{array}$} \\
\hline & & & & ${ }^{3} \mathrm{He}: 0.82$ & n: $2.45^{*}$ \\
\hline${ }^{2} \mathrm{H}+{ }^{3} \mathrm{H} \rightarrow{ }^{4} \mathrm{He}+\mathrm{n}$ & ${ }^{3} \mathrm{H}(\mathrm{d}, \mathrm{n}){ }^{4} \mathrm{He}$ & +17.589 & NA & ${ }^{4} \mathrm{He}: 3.54$ & n: 14.05 \\
\hline \multirow[t]{2}{*}{${ }^{1} \mathrm{H}+{ }^{7} \mathrm{Li} \rightarrow{ }^{7} \mathrm{Be}+\mathrm{n}$} & ${ }^{7} \operatorname{Li}(\mathrm{p}, \mathrm{n})^{7} \mathrm{Be}^{\dagger}$ & -1.644 & 1.880 & ${ }^{7} \mathrm{Be}: 0.21$ & $\mathrm{n}: 0.03$ \\
\hline & ${ }^{1} \mathrm{H}\left({ }^{7} \mathrm{Li}, \mathrm{n}\right){ }^{7} \mathrm{Be}^{\dagger}$ & -1.644 & 13.094 & ${ }^{7} \mathrm{Be}: 10.0$ & $\mathrm{n}: 1.44$ \\
\hline${ }^{2} \mathrm{H}+{ }^{7} \mathrm{Li} \rightarrow{ }^{8} \mathrm{Be}+\mathrm{n}$ & ${ }^{7} \mathrm{Li}(\mathrm{d}, \mathrm{n}){ }^{8} \mathrm{Be}$ & +15.031 & NA & ${ }^{8} \mathrm{Be}: 1.68$ & $\mathrm{n}: 13.35$ \\
\hline${ }^{1} \mathrm{H}+{ }^{9} \mathrm{Be} \rightarrow{ }^{9} \mathrm{~B}+\mathrm{n}$ & ${ }^{9} \mathrm{Be}(\mathrm{p}, \mathrm{n}){ }^{9} \mathrm{~B}$ & -1.850 & 2.057 & ${ }^{9} \mathrm{~B}: 0.18$ & $\mathrm{n}: 0.023$ \\
\hline${ }^{2} \mathrm{H}+{ }^{9} \mathrm{Be} \rightarrow{ }^{10} \mathrm{Be}+\mathrm{n}$ & ${ }^{9} \mathrm{Be}(\mathrm{d}, \mathrm{n}){ }^{10} \mathrm{~B}$ & +4.361 & NA & ${ }^{10} \mathrm{~B}: 0.40$ & n: 3.96 \\
\hline
\end{tabular}

In principle a complete understanding of the direction and kinetic energy of the daughter products of the reactions listed in Table 2 may be established with knowledge of the energy and momentum of the starting nuclei. A useful relationship for determining the energy of the neutrons produced in these reactions, dependent on the energy of the incident particle, is shown in Eq. (2).[22] Referencing the reaction shorthand 2(1,3)4 where particle 1 is accelerated into a stationary target of material 2 to produce the daughter products 3 (a neutron) and $4, \mathrm{E}_{1}$ represents the energy of the accelerated particle, $\mathrm{E}_{3}$ represents the energy of the neutron, $\mathrm{M}_{1}$ is the mass of the accelerated particle, $\mathrm{M}_{3}$ is the mass of the neutron, $\mathrm{M}_{4}$ is the mass of the associated daughter particle, and $\mathrm{Q}$ is the reaction energy, positive for exothermic reactions and negative for endothermic reactions. The direction of the accelerated particle towards stationary particle 2 is used to define the angle of emission of the neutron, $\theta$, after the reaction occurs. This relationship is plotted for the six reactions in Fig. 2 for neutrons emitted in the same direction as the incident particles $\left(\theta=0^{\circ}\right)$ and for neutrons emitted back towards the origin of the incident particles $\left(\theta=180^{\circ}\right)$.

$$
E_{3}^{1 / 2}=\frac{\left(M_{1} M_{3} E_{1}\right)^{1 / 2} \cos \theta \pm\left\{M_{1} M_{3} E_{1} \cos ^{2} \theta+\left(M_{3}+M_{4}\right)\left[M_{4} Q+\left(M_{4}-M_{1}\right) E_{1}\right]\right\}^{1 / 2}}{M_{3}+M_{4}}
$$




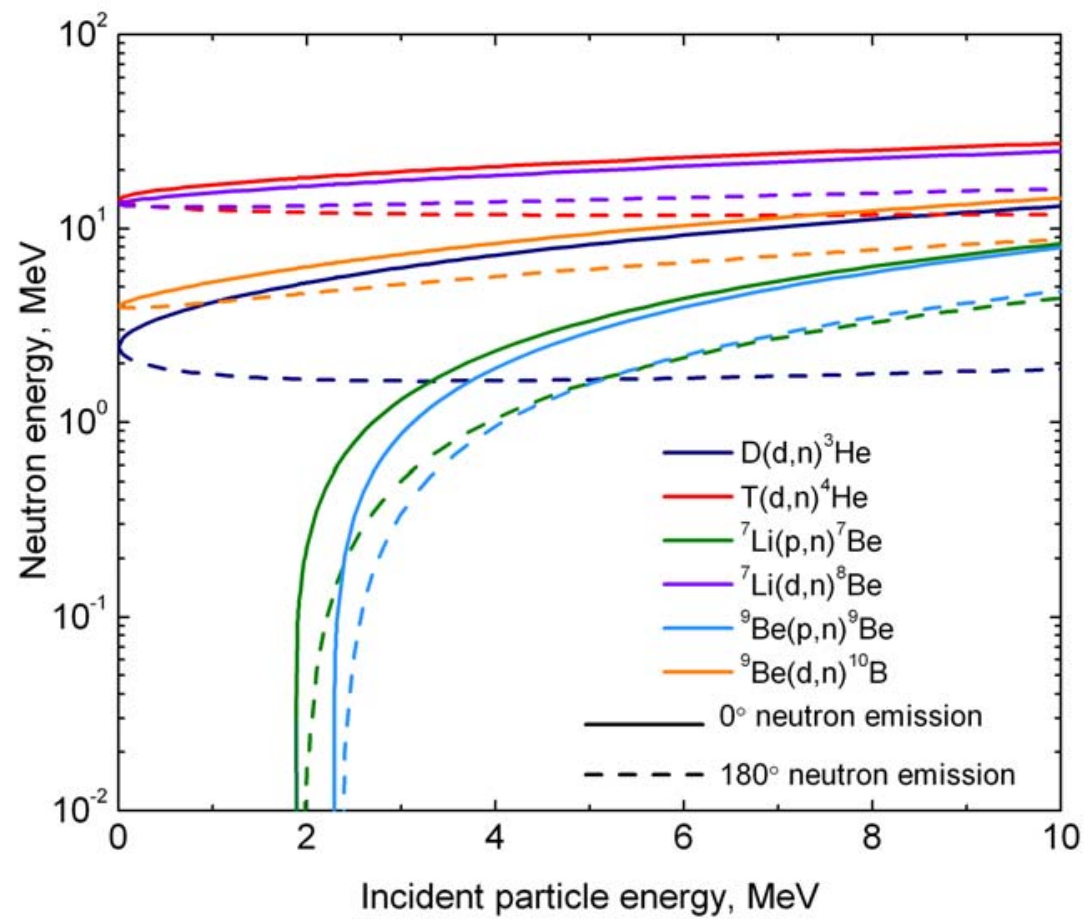

Fig. 2 This plot shows the energy of neutrons produced from several different reactions $X(p, n) Y$ for varying incident particle ( $\mathrm{p}$ or $\mathrm{d}$ ) kinetic energies for neutron emission at $0^{\circ}$ and $180^{\circ}$ with respect to the incident particle. Note: this does not show the probability that the reaction will take place, only the resultant neutron production if it does occur. Also, these neutron energies are theoretical estimates for infinitely thin targets. In thick targets neutrons will be produced in a continuum from the maximum energy down to the minimum energy for each case.

For exothermic reactions only one neutron energy is possible at each observation angle for a particular incident particle energy, and neutrons may be produced for all incident particle energies. However, the probability the neutron producing reactions will occur is not equal for all incident particle energies and Coulombic repulsion generally makes the reaction probabilities vanishingly small for low incident-particle kinetic energies. In endothermic reactions the incident particle energy must exceed a threshold value $\mathrm{E}_{1}^{\text {th }}$ before the reaction becomes possible; the relation for $E_{1}^{\text {th }}$ is defined in Eq. (3). For endothermic reactions if the incident-particle energy exceeds $E_{1}^{\text {th }}$ but is less than a second critical value, $E_{1}^{\prime}$ (Eq. (4)), then two neutron exit energies are possible for a particular emission angle, thus the meaning of the \pm symbol in Eq. (2).[22] The double-valued neutron energy phenomenon exists only for endothermic reactions, reflecting the 
fact that, until the velocity of the neutron in a center-of-mass coordinate system meets or exceeds the velocity of the coordinate system itself, forward going neutrons in the center of mass system neutrons may be observed in a backwards direction in a laboratory coordinate system.[16] This effect is shown in Fig. 3 for the ${ }^{7} \mathrm{Li}(\mathrm{p}, \mathrm{n})^{7} \mathrm{Be}$ reaction.

$$
\begin{gathered}
\mathrm{E}_{1}^{\text {th }}=\frac{-\mathrm{Q}\left(\mathrm{M}_{3}+\mathrm{M}_{4}\right)}{\mathrm{M}_{3}+\mathrm{M}_{4}-\mathrm{M}_{1}} \\
\mathrm{E}_{1}^{\prime}=\frac{-\mathrm{QM}_{4}}{\mathrm{M}_{4}-\mathrm{M}_{1}}
\end{gathered}
$$

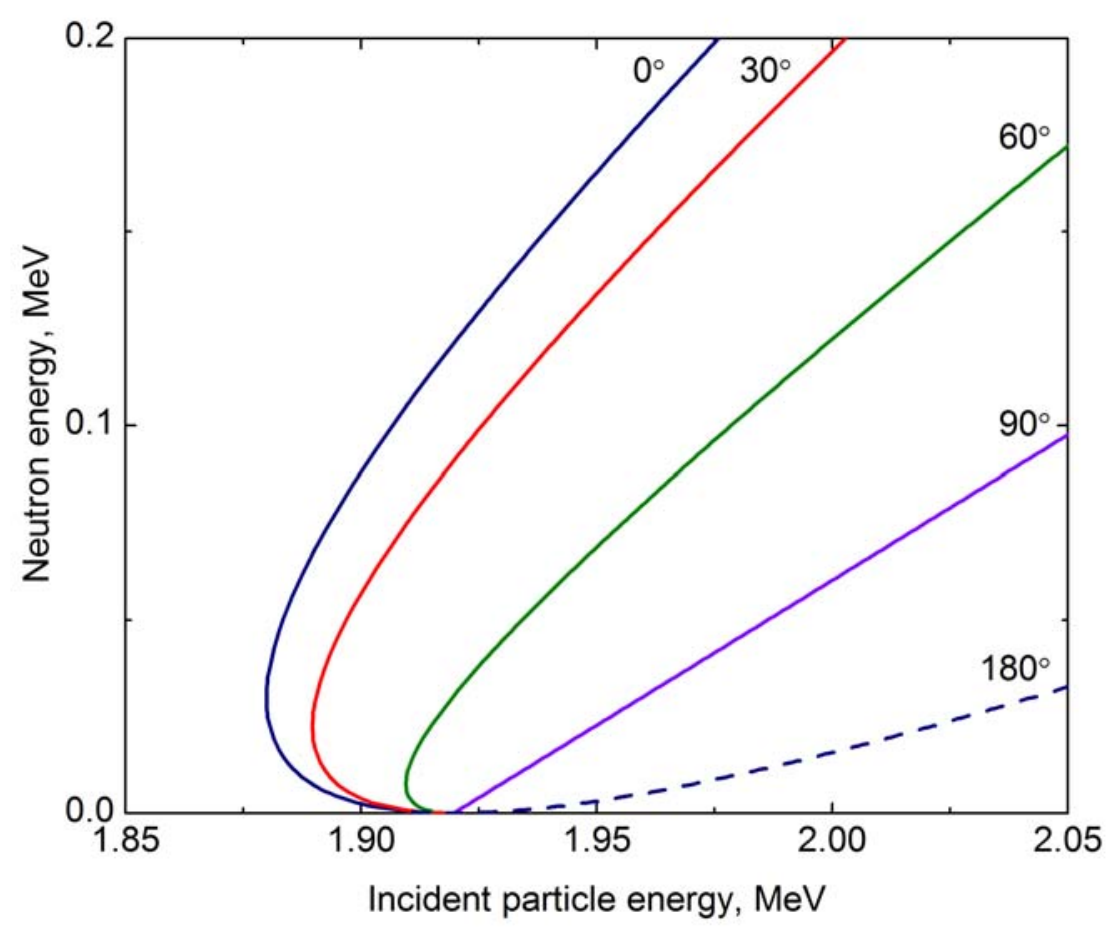

Fig. 3 The low-energy region of the ${ }^{7} \operatorname{Li}(p, n)^{7} \mathrm{Be}$ reaction showing the double-valued nature of the emitted neutron energies for forward directed angles. 
The yield of neutrons produced from these reactions is based on their energy-dependent reaction probabilities, as expressed in their reaction crosssection, $\sigma$. A plot of the reaction cross sections for several of the neutron producing reactions considered in this chapter is shown in Fig. 4. However, in practical applications several other factors also come into play to influence the neutron yield of these reactions generated by accelerators. Within the accelerator as ions impact the target elastic scattering is much more likely than nuclear capture. Because of this, in targets of appreciable thickness there exists a continuum of projectile energies within the target, from very low energies up to the accelerating potential. Known as the thick-target effect, this fact has a significant impact on the observed neutron yielded versus accelerating energy profile for these reactions.

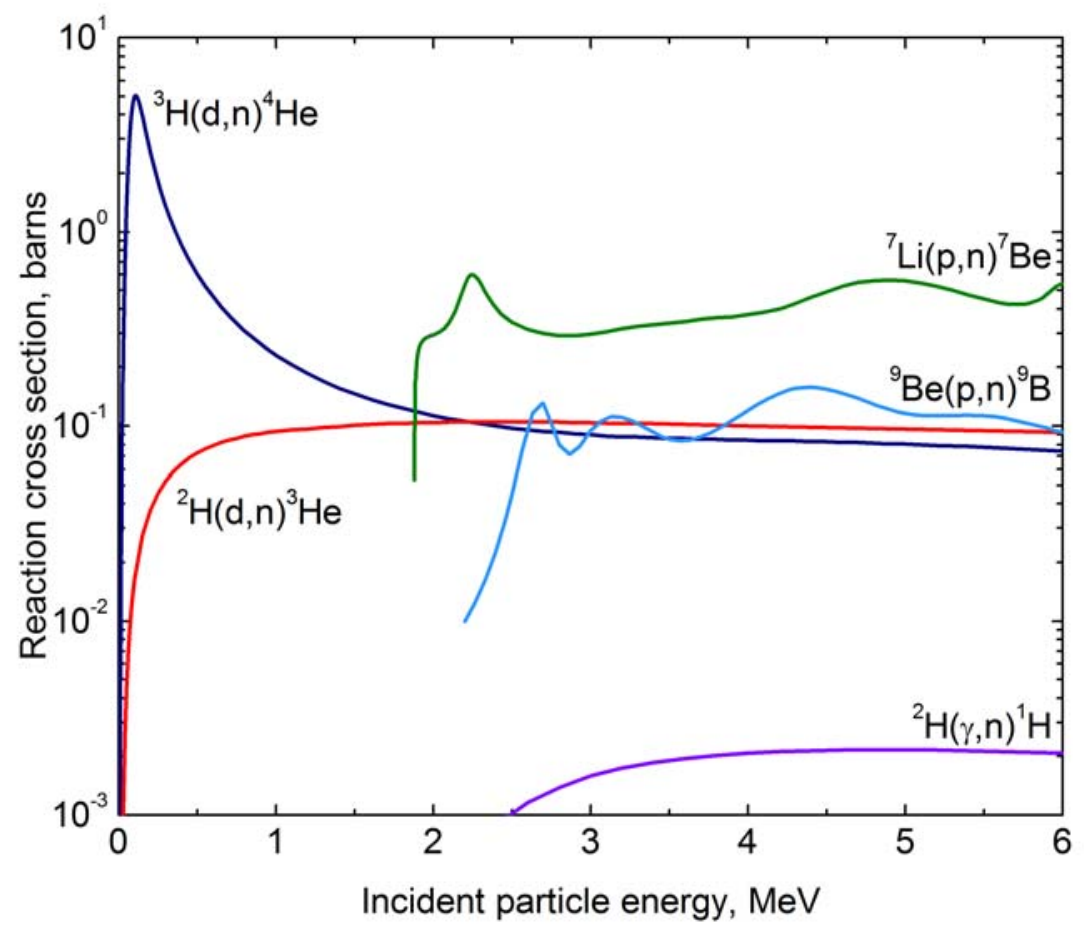

Fig. 4 Reaction cross sections for several accelerator-based neutron producing reactions.[23]

Another important factor influencing the yield versus energy curve for these reactions is whether the accelerated ion beams are of atoms, molecules, or some combination of the two; accelerating both $\mathrm{H}$ and ${ }^{2} \mathrm{H}$ ions of hydrogen are possible. In nearly all cases some fraction of both species is present, with more 
atomic species present when hotter plasma sources are used (e.g. radiofrequency ion sources) and more molecular ion species present when cooler temperature ion sources are used (e.g. cold-cathode sources). Upon hitting the target the interaction of molecular ions may be approximated by considering the ion to split into its constituent atoms, with each atom possessing a fraction of the accelerating potential energy inversely proportional to the ratio of its mass to the molecules mass. Thus, if a molecule of two ${ }^{2} \mathrm{H}$ atoms is accelerated to $100 \mathrm{keV}$ and then impacts a target it may be approximated as two separate ${ }^{2} \mathrm{H}$ atoms each having a kinetic energy of $50 \mathrm{keV}$. Reviewing the plots of Fig. 2 it is clear that at a particular emission angle two different neutron energies will be present, the intensity of which will depend upon the atomic/molecular ratio of the ion beam. A third factor that can influence the neutron yield is the target matrix and its structure.[24] With deuterium and tritium reactions, for example, it is common to use a hydriding metal occluder such as titanium, zirconium, or erbium which can store hydrogen within their lattice structures. These different targets can have different densities depending upon how they're created and they can store different concentrations of the hydrogen isotopes depending on several factors including preparation and temperature. A generic example of actual neutron yield profiles for several reactions is shown in Fig. 5.[25] 


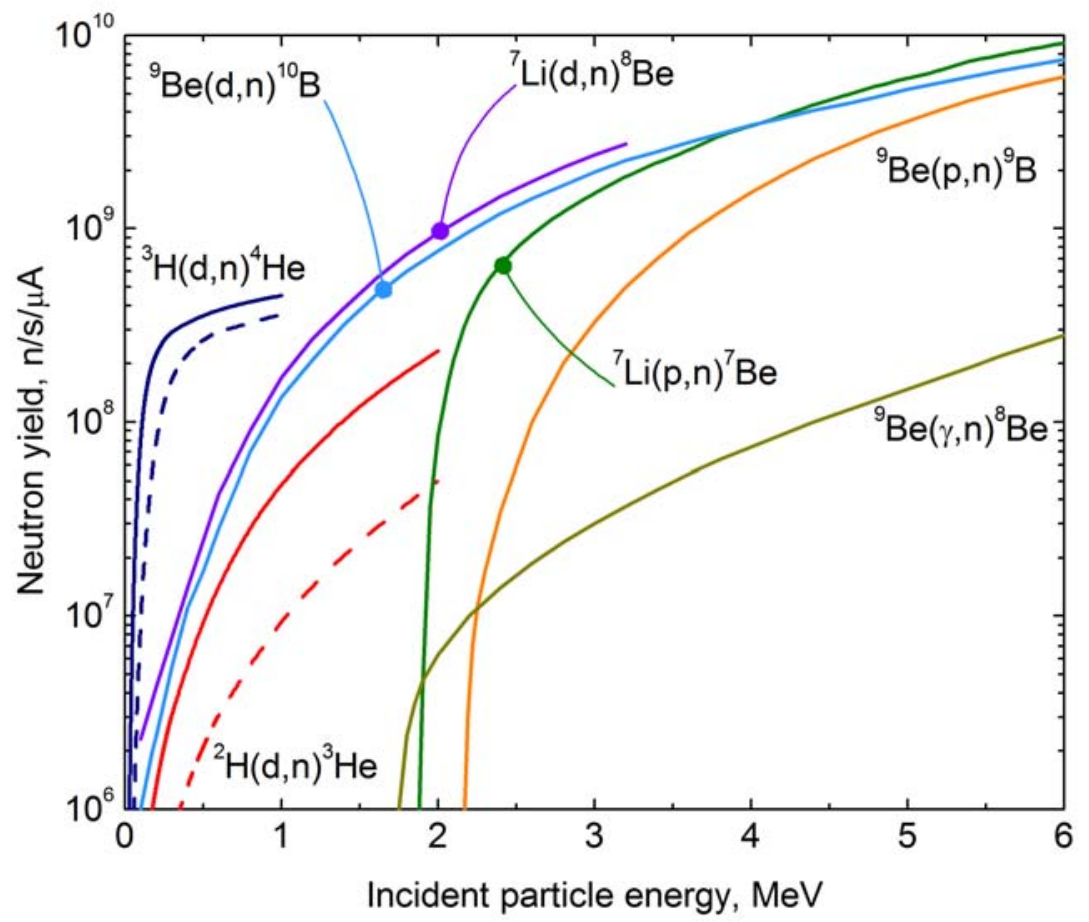

Fig. 5 Total neutron yield curves for several reactions. (The DD and DT data for this plot is derived from reference 24 and refers to a fully loaded titanium target with deuterons accelerated into $\mathrm{TiD}_{2}$ for the ${ }^{2} \mathrm{H}(\mathrm{d}, \mathrm{n})^{3} \mathrm{He}$ reaction and deuterons accelerated into $\mathrm{TiT}_{2}$ for the ${ }^{3} \mathrm{H}(\mathrm{d}, \mathrm{n})^{4} \mathrm{He}$. The solid plots refer to $100 \%$ atomic beams, except for the ${ }^{9} \mathrm{Be}(\gamma, \mathrm{n})^{8} \mathrm{Be}$ reaction, while the two dashed plots refer to $100 \%$ molecular ion beams.)[24,25]

\subsection{1 ${ }^{2} \mathrm{H}+{ }^{2} \mathrm{H} \rightarrow{ }^{3} \mathrm{He}+\boldsymbol{n}$}

Using the DD fusion reaction is a straightforward and often used technique for neutron production with particle accelerators. The reaction is exothermic and its cross section yields a useful neutron production rate with accelerating energies of $\mathcal{O}(100) \mathrm{keV}$ and modest beam currents of $\mathcal{O}(100) \mu \mathrm{A}$. Neutrons from this reaction start at $\sim 2.5 \mathrm{MeV}$, they are roughly monoenergetic at lower accelerating energies but less so at higher energies, as shown in Fig. 6. The low $\mathrm{Q}$ value for the reaction results in a forward-directed anisotropic yield even at low accelerating potentials, with the relative yield from $0^{\circ}$ to $90^{\circ}$ decreasing by $4 \times$ for an accelerating potential $0.5 \mathrm{MeV}$.[19] The low yield of this reaction compared with the others described here limits its use in many 
applications but is sometimes compensated by the lower energy of DD neutrons versus those from DT fusion or other reactions.

The cross section for DD fusion is approximately the same as the competing reaction ${ }^{2} \mathrm{H}+{ }^{2} \mathrm{H} \rightarrow{ }^{3} \mathrm{H}+{ }^{1} \mathrm{H}$. This has proven to be a problem in some high-yield, sealed-tube electronic neutron generators due to the build-up of tritium in the system which eventually leads to the introduction of higher energy neutron contamination from the DT reaction. Indeed, in one high-yield system using a sealed DD neutron tube the yield of DT fusion neutrons increased to onetenth the DD yield after only 1000 hours of operation.[26] One further complication can arise at higher accelerating energies due to the ${ }^{2} \mathrm{H}(\mathrm{d}, \mathrm{np})^{2} \mathrm{H}$ breakup reaction, which occurs when the deuteron energy exceeds $4.45 \mathrm{MeV}$, introducing a second energy group to the neutron spectrum for this reaction.

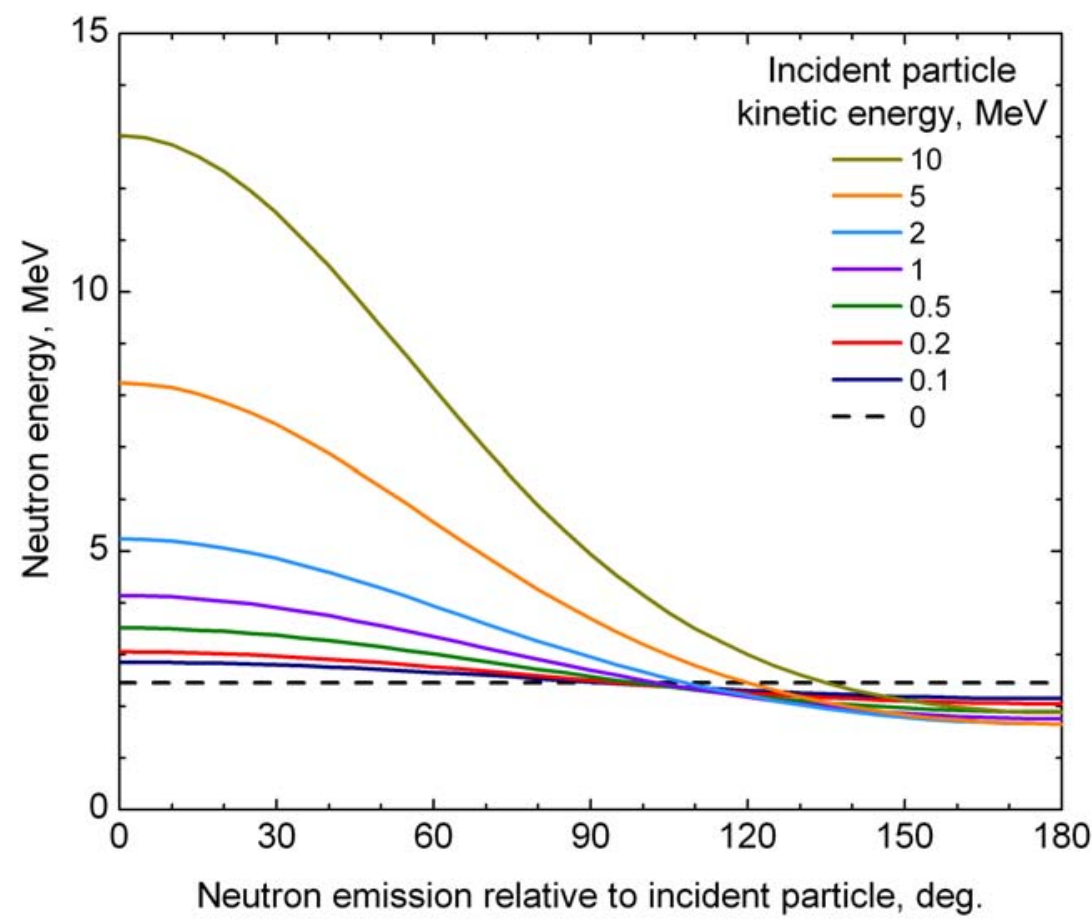

Fig. 6 The theoretical energy of neutrons from the ${ }^{2} \mathrm{H}(\mathrm{d}, \mathrm{n})^{3} \mathrm{He}$ fusion reaction at different emission angles for varying incident particle kinetic energies. 


\subsection{2 ${ }^{2} \mathrm{H}+{ }^{3} \mathrm{H} \rightarrow{ }^{4} \mathrm{He}+\boldsymbol{n}$}

The DT fusion reaction is the most often used nuclear reaction for producing neutrons with accelerators. It has the highest neutron yield for low accelerating voltages, less than $1 \mathrm{MeV}$, and produces roughly monoenergetic neutrons at accelerating potentials $(\mathcal{O}(100) \mathrm{keV})$ and beam currents $(\mathcal{O}(100) \mu \mathrm{A})$ typically used with electronic neutron generators, as seen in Fig. 7. The high Q value makes DT accelerator neutron sources a close approximation for isotopic sources at low accelerating energies.[19] In sealed-vacuum accelerator systems the presence of both deuterium and tritium can introduce some complexity to the neutron spectrum; the use of molecular beams ion sources can further complicate the spectrum. As with the DD reaction a competing breakup reaction exists with DT fusion too, occurring for deuteron energies exceeding $3.71 \mathrm{MeV}$ and adding a second neutron group to the output spectrum.

Historically the presence of tritium in accelerator systems introduced challenges related to radiation safety and contamination control; this has limited the use of tritium in commercial open vacuum-accelerator systems.[27,28] For example, the ${ }^{3} \mathrm{H}(\mathrm{p}, \mathrm{n})^{3} \mathrm{He}$ reaction is often viewed as a useful neutron producing reaction together with the other reactions presented in this chapter. However, the reaction requires a minimum accelerating potential of $1.02 \mathrm{MeV}$, eliminating it from practical consideration in current-generation sealed-vacuum accelerator systems. Requiring an open-vacuum system, therefore, the hazards associated with tritium contamination impose a steep barrier on this reaction's implementation in practical commercial systems. In sealed-vacuum systems tritium leakage is still found to occur but it has not proven to be a hurdle for commercialization. 


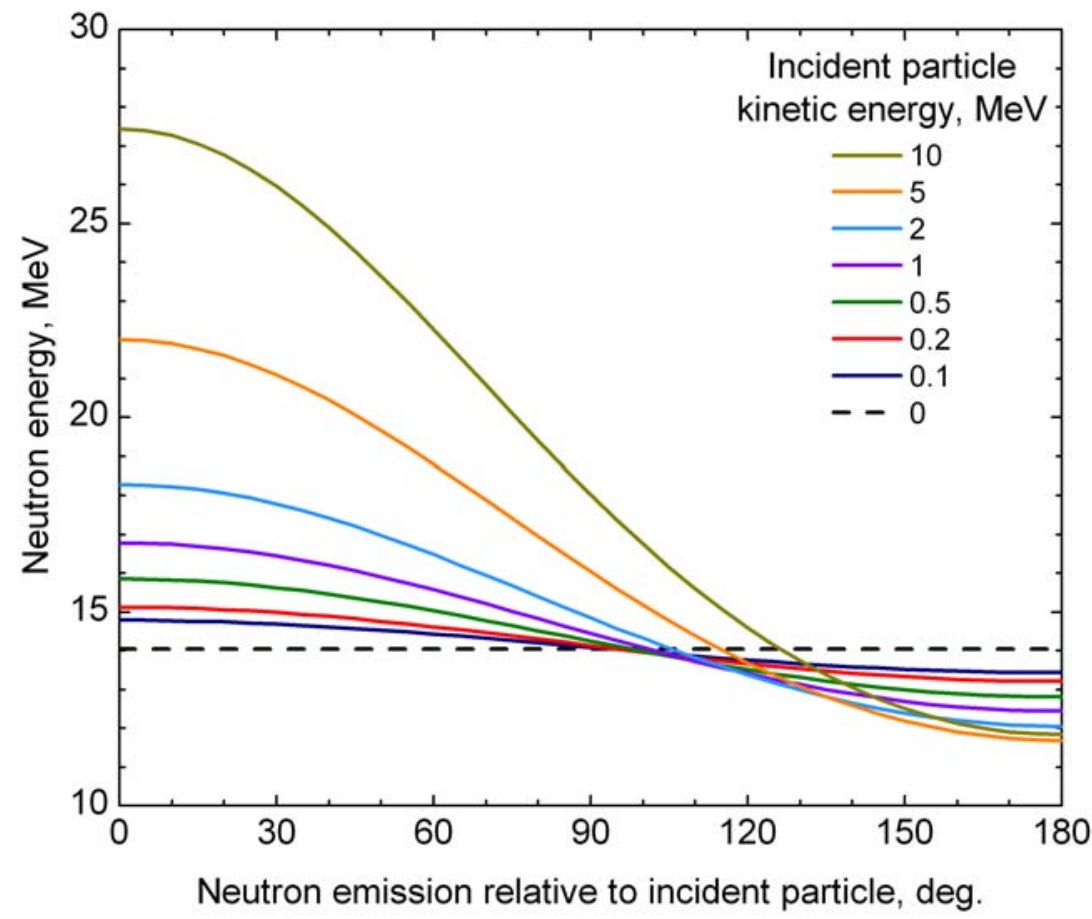

Fig. 7 The theoretical energy of neutrons from the ${ }^{3} \mathrm{H}(\mathrm{t}, \mathrm{n})^{4} \mathrm{He}$ fusion reaction at different emission angles for varying incident particle kinetic energies.

\subsection{3 ${ }^{7} \mathrm{Li}+{ }^{1} \mathrm{H} \rightarrow{ }^{7} \mathrm{Be}+n$}

The forward focused, relatively low energy neutron field produced with the ${ }^{7} \mathrm{Li}(\mathrm{p}, \mathrm{n})^{7} \mathrm{Be}$ reaction has been the motivation for considering its use in many commercial applications. As shown in Fig. 8, when protons only slightly higher in energy (e.g., $1.89 \mathrm{MeV})$ than the reaction's threshold energy $(1.88 \mathrm{MeV})$ are accelerated towards ${ }^{7} \mathrm{Li}$ neutrons less than $0.1 \mathrm{MeV}$ are produced, limited to an emission cone of $60^{\circ}$. This results in an improvement in efficiency over isotropic neutron sources by allowing more neutrons to be directed towards a target area in interrogation applications; the forward-directed beam also helps in limiting backangle radiation dose fields, which can reduce shielding requirements when using this reaction. The reaction produces neutrons of two energies at small angles and, as mentioned earlier, in thick targets a continuum of lower-energy neutrons is present as the incident ions lose energy through their transit of the target (a good presentation of expected neutron spectra from this reaction may be found 
on pages 40-44 of reference 29). The energy of the first excited state of ${ }^{7} \mathrm{Be}$ is $0.429 \mathrm{MeV}$. If the incident proton energy exceeds $2.37 \mathrm{MeV}$ it is possible for the residual ${ }^{7} \mathrm{Be}$ nucleus to be left in this higher energy state, ${ }^{7} \mathrm{Li}(\mathrm{p}, \mathrm{n})^{7} \mathrm{Be}^{*}$. When this reaction pathway becomes possible the energy spectrum of neutrons from the reaction becomes more complicated and includes the primary group, as read from Fig. 8, as well as lower energy neutrons generated as if the kinetic energy of the incident proton was $\mathrm{E}_{\mathrm{p}}-0.429 \mathrm{MeV}$. Additional complications in the neutron spectrum occur as the energy is increased above $3.69 \mathrm{MeV}$, with the introduction of the ${ }^{7} \mathrm{Li}\left(\mathrm{p}, \mathrm{n}^{3} \mathrm{He}\right)^{4} \mathrm{He}$ reaction channel, and above $7.11 \mathrm{MeV}$ with the opening of a reaction channel for exciting the $4.57-\mathrm{MeV}$ level of ${ }^{7} \mathrm{Be}$. An engineering challenge associated with using this reaction is the design of a target system that can sufficiently cool a lithium target without allowing a high power ion beam to cause damage.[30]

A notable variation for using this reaction is its implementation when ${ }^{7} \mathrm{Li}$ ions are accelerated into a hydrogen-bearing target.[31,32] For an accelerating potential of $13.5 \mathrm{MeV}$, only slightly above the $13.07 \mathrm{MeV}$ threshold, the reaction cross section reaches 4 barns per steradian and all neutrons emissions are limited to a $9^{\circ}$ cone. The potential advantages of having a highly focused fast neutron beam, as shown in Fig. 8, are numerous but the technical hurdles towards achieving a practical accelerating system are similarly high. (Again, at higher accelerating energies excitation of the first excited state of ${ }^{7} \mathrm{Be}$ can complicate the emissions of this reaction.) Industrial cyclotron systems are routinely used at the higher energies needed for this reaction but their implementation for use in industrial (versus medical) instrument environments has not yet been realized. Also, the instrumentation needed to create ${ }^{7} \mathrm{Li}$ ions is more complicated then that need for creating hydrogen ions and has not yet been developed for high reliability operations. 

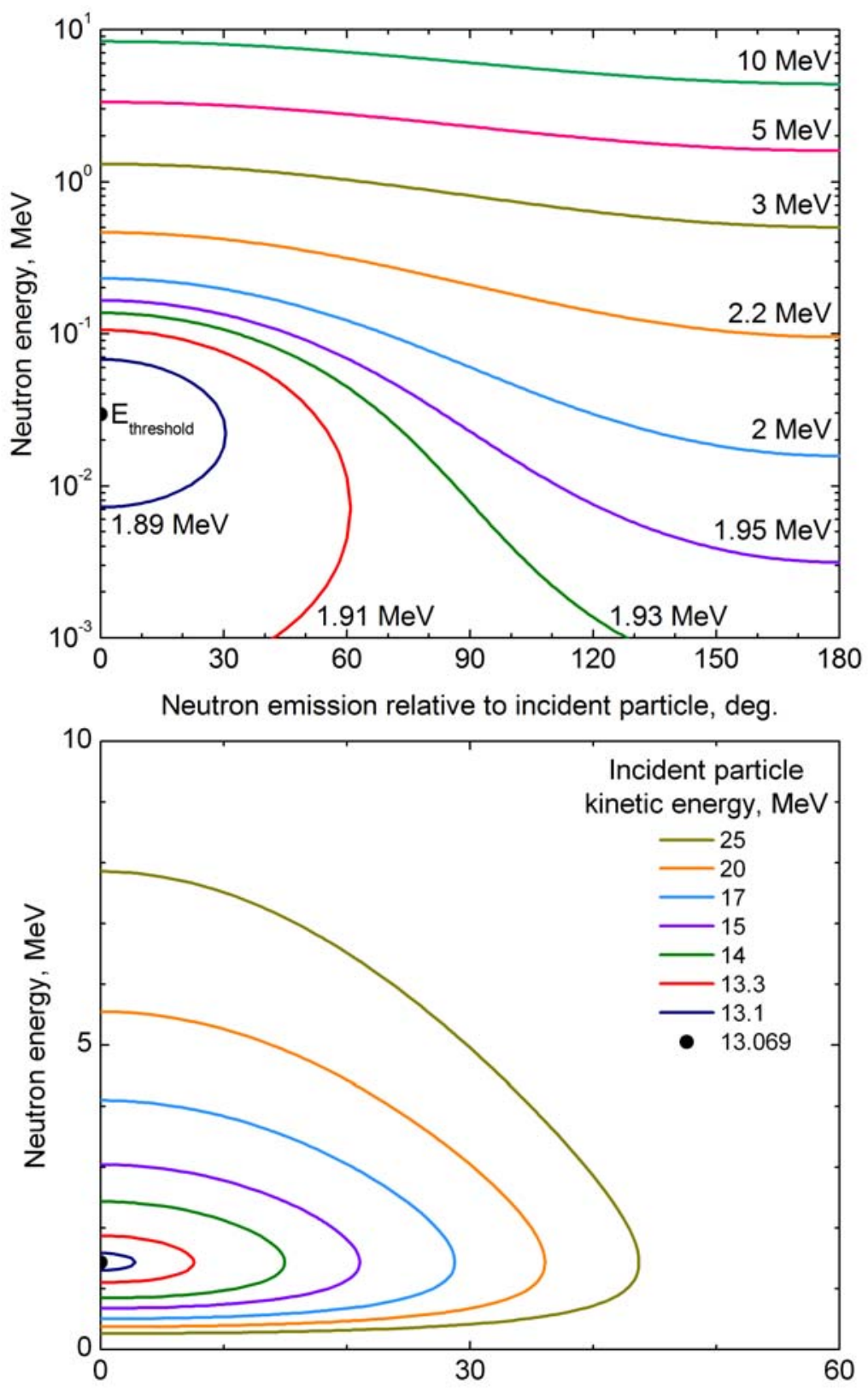

Neutron emission relative to incident particle, deg.

Fig. 8 The theoretical energy of neutrons from the ${ }^{7} \mathrm{Li}(\mathrm{p}, \mathrm{n})^{7} \mathrm{Be}$ fusion reaction (top) and the ${ }^{1} \mathrm{H}\left({ }^{7} \mathrm{Li}, \mathrm{n}\right){ }^{7} \mathrm{Be}$ fusion reaction (bottom) at different emission angles for varying incident particle kinetic energies. 


\subsection{4 ${ }^{7} \mathrm{Li}+{ }^{2} \mathrm{H} \rightarrow{ }^{8} \mathrm{Be}+n$}

Rather than using protons another method for producing neutrons with a ${ }^{7} \mathrm{Li}$ target is to use deuterons. An exothermic reaction with a high $\mathrm{Q}$ value, the ${ }^{7} \mathrm{Li}(\mathrm{d}, \mathrm{n})^{8} \mathrm{Be}$ reaction is similar to the DT reaction and at lower accelerating energies it produces a generally monoenergetic $( \pm 0.5 \mathrm{MeV})$, high-energy neutron spectrum in all emission angles, as shown in Fig. 9. However, the cross section for the reaction increases less strongly with energy than for the DT reaction and accelerating energies up to $1 \mathrm{MeV}$ are needed to approach comparable neutron yields (Fig. 5). When high energy neutrons are needed this reaction presents an attractive alternative to the DT fusion source that avoids the need for dealing with and handling tritium. Of course, the same target cooling challenges are present with this reaction as with the ${ }^{7} \mathrm{Li}+\mathrm{p}$ reaction.

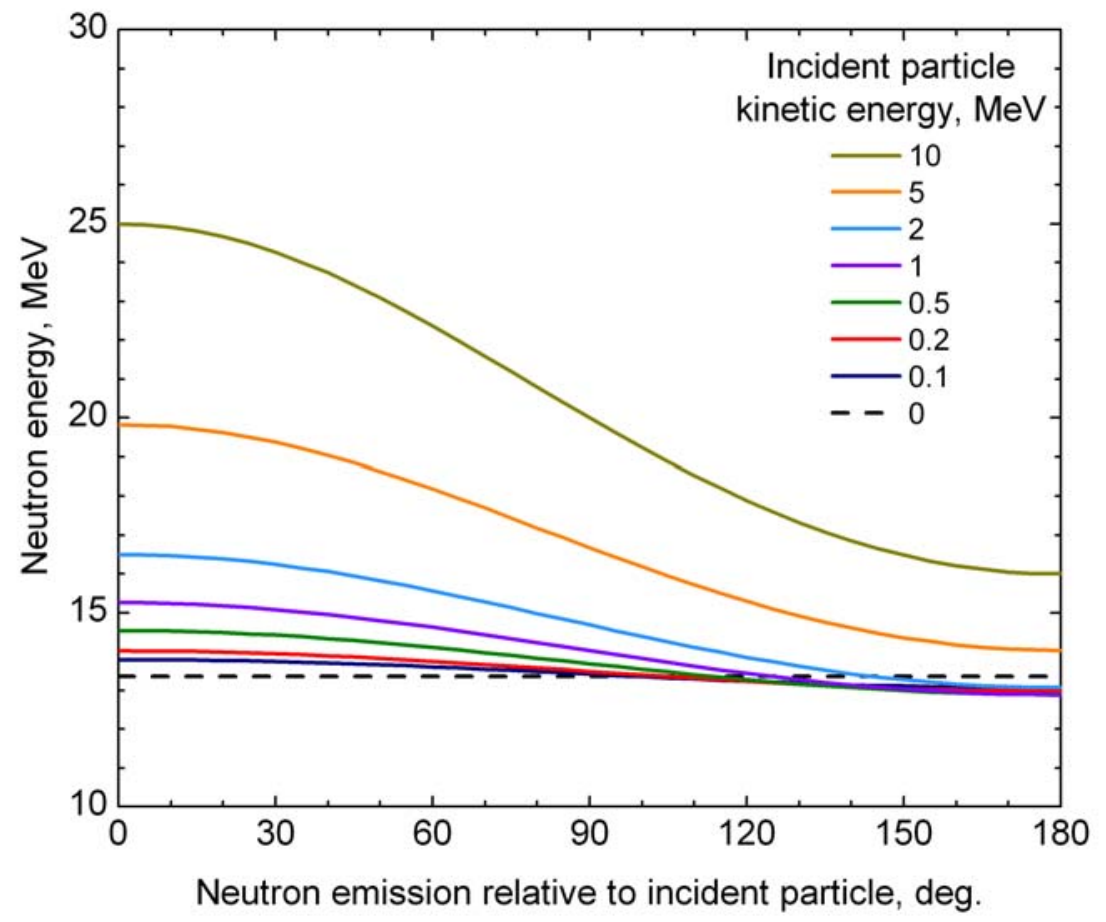

Fig. 9 The theoretical energy of neutrons from the ${ }^{7} \mathrm{Li}(\mathrm{d}, \mathrm{n})^{8} \mathrm{Be}$ fusion reaction at different emission angles for varying incident particle kinetic energies. 


\subsection{5 ${ }^{9} \mathrm{Be}+{ }^{1} \mathrm{H} \rightarrow{ }^{9} \mathrm{~B}+n$}

Beryllium may be used in place of lithium in particle accelerators to produce neutrons. With protons as the incident accelerated particles neutron production is similar as with lithium targets, the reaction is endothermic with a threshold energy of $2.290 \mathrm{MeV}$. Close to this threshold the reaction emits neutrons in a forward direction and is double valued, as shown in Fig. 10. Slightly higher accelerating energies are needed with beryllium targets in comparison with lithium targets, the neutron yields are less under comparable operating conditions. One notable advantage with the use of beryllium is its superior mechanical properties to handle high power densities and incorporate forced cooling. As with the lithium target reaction ion straggling within the beryllium target produces a continuum of neutron energies below the peak energy as a result of ion energy losses in the beryllium target.

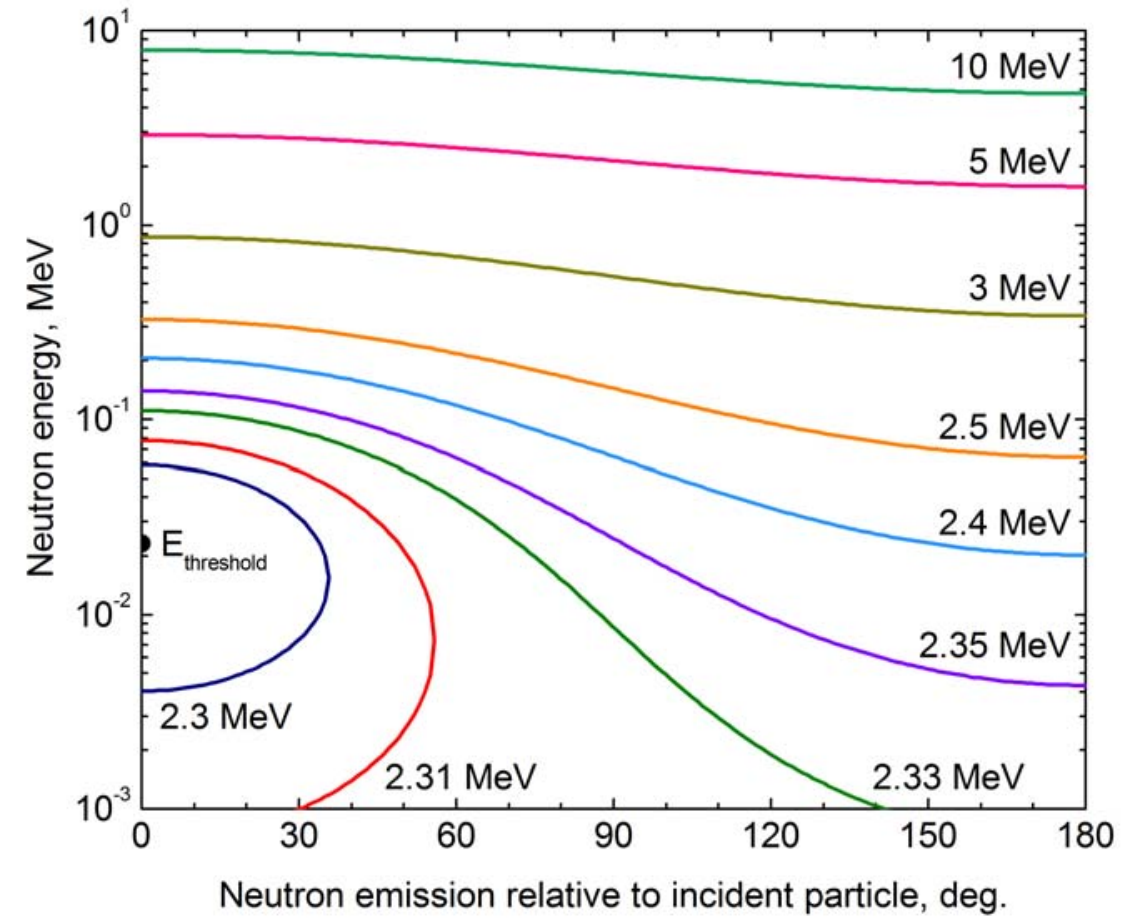

Fig. 10 The theoretical energy of neutrons from the ${ }^{9} \mathrm{Be}(\mathrm{p}, \mathrm{n})^{9} \mathrm{~B}$ fusion reaction at different emission angles for varying incident particle kinetic energies. 


\subsection{6 ${ }^{9} \mathrm{Be}+{ }^{2} \mathrm{H} \rightarrow{ }^{10} \mathrm{~B}+\boldsymbol{n}$}

Rounding out the most commonly used accelerator-based neutron producing reactions is the ${ }^{9} \mathrm{Be}(\mathrm{d}, \mathrm{n}){ }^{10} \mathrm{~B}$ reaction. Mirroring the similarities between lithium and beryllium targets with protons, the neutron yield from this exothermic reaction has a similar energy dependence as with lithium. However, with a $\mathrm{Q}$ value roughly one-third that with lithium the neutron spectra from this reaction starts near $4 \mathrm{MeV}$, as seen in Fig. 11. In some cases this lower-energy neutron output, which is again a close approximation of a monoenergetic, isotropic neutron source at low energies, could be a desirable characteristic.

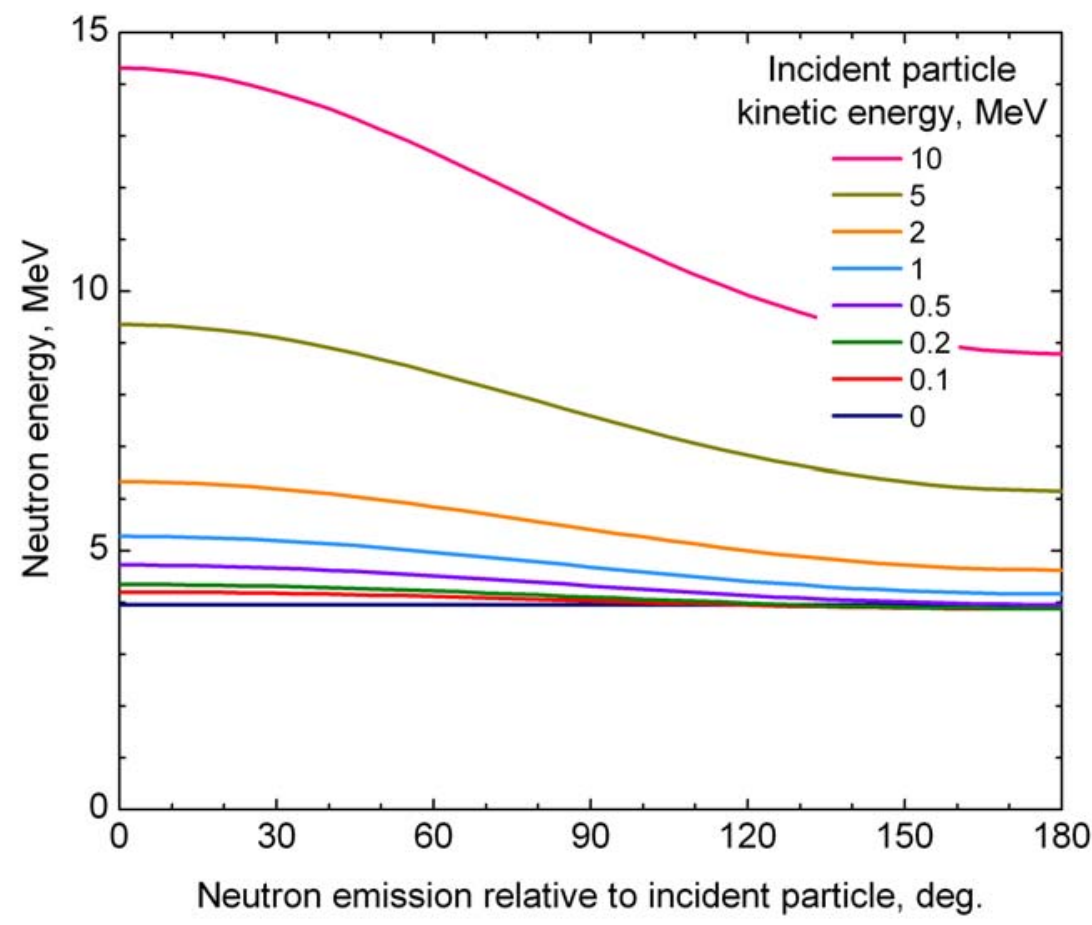

Fig. 11 The theoretical energy of neutrons from the ${ }^{9} \mathrm{Be}(\mathrm{d}, \mathrm{n}){ }^{10} \mathrm{~B}$ fusion reaction at different emission angles for varying incident particle kinetic energies.

\subsubsection{Other Two-Body Ion Reactions}

Beside the light ion reactions listed above there are many other neutron producing reactions with favorable characteristics for industrial applications, a selection of these reactions is presented in Table 3. These reactions are capable 
of generating neutrons at several different energies, with varying monoenergetic characteristics, and depending upon particle-target choices, varying degrees of forward focusing. The first reaction, using tritium, is perhaps the most versatile in this list.[33] When used with thin targets (gas targets) the ${ }^{1} \mathrm{H}+{ }^{3} \mathrm{H}$ reaction can generate near-monoenergetic neutrons over a wide energy range. However, its negative $\mathrm{Q}$ value removes the reaction from consideration in traditional lowvoltage, vacuum-sealed electronic neutron generators, and the subsequent need for a tritium handling infrastructure in an open-vacuum system adds an extra level of complexity not readily accepted in commercial systems.

Table 3 A partial list of additional charged particle reactions potentially useful in industrial applications. [34,35]

\begin{tabular}{ccc}
\hline Reaction & $\begin{array}{c}\text { Q Value } \\
{[\text { MeV }]}\end{array}$ & $\begin{array}{c}\text { Threshold Energy } \\
{[\text { MeV }]}\end{array}$ \\
\hline${ }^{3} \mathrm{H}+{ }^{1} \mathrm{H} \rightarrow{ }^{3} \mathrm{He}+\mathrm{n}$ & -0.764 & 1.019 \\
${ }^{3} \mathrm{H}+{ }^{3} \mathrm{H} \rightarrow{ }^{4} \mathrm{He}+2 \mathrm{n}$ & 11.332 & $\mathrm{NA}$ \\
${ }^{6} \mathrm{Li}+{ }^{1} \mathrm{H} \rightarrow{ }^{6} \mathrm{Be}+\mathrm{n}$ & -5.071 & 5.920 \\
${ }^{6} \mathrm{Li}+{ }^{2} \mathrm{H} \rightarrow{ }^{7} \mathrm{Be}+\mathrm{n}$ & 3.381 & $\mathrm{NA}$ \\
${ }^{10} \mathrm{~B}+{ }^{1} \mathrm{H} \rightarrow{ }^{10} \mathrm{C}+\mathrm{n}$ & -4.430 & 4.876 \\
${ }^{10} \mathrm{~B}+{ }^{2} \mathrm{H} \rightarrow{ }^{11} \mathrm{C}+\mathrm{n}$ & 6.465 & $\mathrm{NA}$ \\
${ }^{11} \mathrm{~B}+{ }^{1} \mathrm{H} \rightarrow{ }^{11} \mathrm{C}+\mathrm{n}$ & -2.765 & 3.018 \\
${ }^{11} \mathrm{~B}+{ }^{2} \mathrm{H} \rightarrow{ }^{12} \mathrm{C}+\mathrm{n}$ & 13.732 & $\mathrm{NA}$ \\
${ }^{12} \mathrm{C}+{ }^{1} \mathrm{H} \rightarrow{ }^{12} \mathrm{~N}+\mathrm{n}$ & -18.121 & 19.642 \\
${ }^{12} \mathrm{C}+{ }^{2} \mathrm{H} \rightarrow{ }^{13} \mathrm{~N}+\mathrm{n}$ & -0.281 & 0.328 \\
${ }^{13} \mathrm{C}+{ }^{1} \mathrm{H} \rightarrow{ }^{13} \mathrm{~N}+\mathrm{n}$ & -3.003 & 3.235 \\
${ }^{13} \mathrm{C}+{ }^{2} \mathrm{H} \rightarrow{ }^{14} \mathrm{~N}+\mathrm{n}$ & 5.326 & $\mathrm{NA}$ \\
${ }^{36} \mathrm{Cl}+{ }^{1} \mathrm{H} \rightarrow{ }^{36} \mathrm{Ar}+\mathrm{n}$ & -0.073 & 0.075 \\
${ }^{51} \mathrm{~V}+{ }^{1} \mathrm{H} \rightarrow{ }^{51} \mathrm{Cr}+\mathrm{n}$ & -1.535 & 1.565 \\
\hline
\end{tabular}

The ${ }^{1} \mathrm{H}+{ }^{3} \mathrm{H}$ and other reactions in this table, most notably the proton and deuteron reactions with boron, are all capable of generating useful neutron 
spectra.[36,37] In all cases, and in particular as the mass and complexity of the parent nuclei increases, care must be taken to understand the presence and impact of competing break-up reactions, and the impact of these often undesired reaction channels towards complicating what would otherwise be monoenergetic spectra.[38] Also, as the nuclei become larger the neutron producing reactions begin to be accompanied by high-energy gamma rays, as with the ${ }^{2} \mathrm{H}+{ }^{11} \mathrm{~B}$ reaction which generates $15.09 \mathrm{MeV}$ photons. These effects may be seen as benefits or hindrances, depending upon the application.[39,40]

\subsection{Reactions With Photons}

Rather than using ions in charged-particle reactions to generate neutrons, highenergy photons may be used to produce neutrons with some nuclei via photonuclear $(\gamma, \mathrm{xn})$ or photofission $(\gamma, \mathrm{f})$ reactions. These reactions occur when the incident photons have sufficient energy to overcome the neutron binding energies for the candidate nuclei. A list of commonly used target nuclei and their threshold energies for neutron emissions is shown in Table 4; the most commonly used materials are beryllium and deuterium. To produce these photons electron accelerators, either linear accelerators or betatrons, are the preferred choice. In these cases high-energy electrons are directed towards targets (often made of high- $Z$ materials such as tungsten or tantalum) and, as these electrons scatter and lose energy within the targets, they produce $\mathrm{x}$ rays (bremsstrahlung) in a continuum spectrum extending up to the maximum energy of the electrons entering the targets. Suitable photoneutron targets are then positioned in front of the x-ray convertor targets to produce neutrons. Neutrons produced from these reactions are not monoenergetic but form a continuum in energy, with a maximum equal to the highest photon energy minus the threshold energy for the reaction. 
Table 4 Commonly used target materials for producing neutrons with photons

\begin{tabular}{cc}
\hline Reaction & $\begin{array}{c}\text { Threshold Energy } \\
{[\mathrm{MeV}]}\end{array}$ \\
\hline${ }^{2} \mathrm{H}$ & 2.22 \\
${ }^{6} \mathrm{Li}$ & 5.39 \\
${ }^{7} \mathrm{Li}$ & 7.25 \\
${ }^{9} \mathrm{Be}^{*}$ & 1.66 \\
${ }^{210} \mathrm{~Pb}$ & 5.18 \\
${ }^{235} \mathrm{U}$ & 5.31 \\
${ }^{238} \mathrm{U}$ & 5.08 \\
\hline
\end{tabular}

${ }^{*}$ Multiple breakup reactions occur with the ${ }^{9} \mathrm{Be}$ photonuclear reaction.

The key variables impacting the neutron yield for accelerator systems using these reactions, besides the reaction cross sections themselves, are the beam energy, beam current, x-ray converter target material and thickness, and neutron production target material and thickness. Specific data for neutron production yields is dependent on specific conditions for a particular system; as a general comparison the reaction cross sections for three of these reactions is shown in Fig. 12. In one case using a $5-\mathrm{MeV}$ electron beam, a $1.698 \mathrm{~g} / \mathrm{cm}^{2}$-thick tungsten converter, a $14-\mathrm{cm}$ thick beryllium deuteride $\left(\mathrm{BeD}_{2}\right)$ neutron production target, and an elaborate neutron reflector a thermal neutron flux of $1.23 \times 10^{8}$ $\mathrm{n} / \mathrm{cm}^{2} / \mathrm{mA} / \mathrm{s}$ was reported.[41] In a second case using a $10-\mathrm{MeV}$ electron beam (the bremsstrahlung converter target was not defined), neutron production yields from $\mathrm{LiD}, \mathrm{Be}$, depleted $\mathrm{U}$, and natural $\mathrm{Pb}$ targets were reported to be $5.2 \times 10^{7}$, $5.7 \times 10^{7}, 4.7 \times 10^{7}$, and $8.0 \times 10^{6}$ neutrons per rad of beam dose.[42] (Because of the dependence on the irradiation parameters above, reported photoneutron yields are normalized to the beam dose rather than electron accelerator's beam current.) 


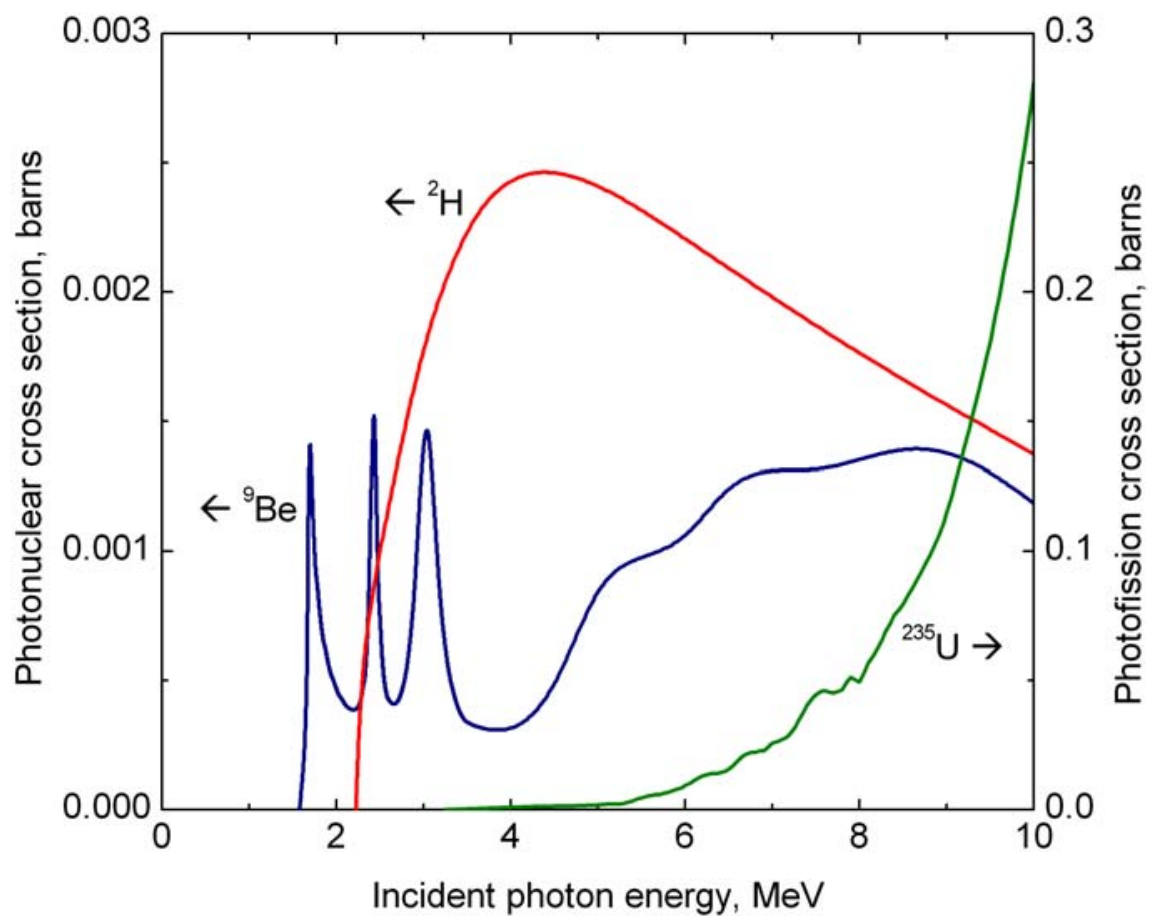

Fig. 12 Photonuclear neutron production cross sections for beryllium and deuterium and the photofission cross section for ${ }^{235} \mathrm{U} .[43]$

One useful capability associated with using pulsed photoneutron sources is the inherent generation of broad-energy, "white spectrum" neutrons and the ability to use these in time-of-flight (ToF) arrangements to carry out energydependent measurements.[44-49] Accelerators systems designed to do this must be capable of generating short pulses with widths of $\mathcal{O}(10)$ ns or less, and typically incorporate beam lines of roughly $5 \mathrm{~m}$ or greater. First, neutrons are generated across a broad energy spectrum simultaneously within a neutron production target, such as beryllium or deuterated water, during the accelerator pulse. These high-energy neutrons travel away from the production target with different velocities, $v$, according to their kinetic energy, $E_{n}$, as shown in the first half of Eq. (5) (neglecting relativistic effects). Rewriting the velocity term in this equation as $L / t$ where $L$ is distance from the neutron production source to a detector (the length of a beam line) and $t$ is the transit time from source to detector, the energy of neutrons measured in a neutron detector is determined by recording the flight time using the accelerator pulse as a starting time, as shown in the second half of Eq. (5). 


$$
\mathrm{E}_{\mathrm{n}}=\frac{1}{2} \mathrm{~m} \mathrm{v}^{2}=\alpha^{2}\left(\frac{L}{t}\right)^{2}
$$

With a system having an infinitely short pulse width the uncertainty associated with the timing measurements would be the primary factor impacting the energy resolution of neutron measurements; finite duration pulses further blur the energy resolution. Using values of $939.6 \mathrm{MeV} / \mathrm{c}^{2}$ for the rest mass of the neutron, $m$, and $2.998 \times 10^{8} \mathrm{~m} / \mathrm{s}$ for $c$, the constant $\alpha$ equals $72.3(\mathrm{eV})^{1 / 2} \cdot(\mu \mathrm{s} / \mathrm{m})$ when the beam length is measured using meters and time is recorded in microseconds, yielding neutron energy in $\mathrm{eV}$. An expression for the neutron energy resolution capable in ToF measurements is shown in Eq. (6). In addition to the minimization of timing uncertainties and reducing pulse widths, longer beam tube distances can also help improve the precision of these measurements, with the drawback of diminishing the overall source intensity on target, which often leads to long measurement times.

$$
\sigma_{\mathrm{E}_{\mathrm{n}}}=2 \mathrm{E}_{\mathrm{n}}\left(\frac{\sigma_{\mathrm{L}}^{2}}{\mathrm{~L}^{2}}+\frac{\sigma_{\mathrm{t}}^{2}}{\mathrm{t}^{2}}\right)^{\frac{1}{2}}=2 \mathrm{E}_{\mathrm{n}}\left(\frac{\sigma_{\mathrm{L}}^{2}}{\mathrm{~L}^{2}}+\frac{\sigma_{\mathrm{t}}^{2}}{\alpha^{2} \mathrm{~L}^{2}} \mathrm{E}_{\mathrm{n}}\right)^{\frac{1}{2}}
$$

A drawback with the use of photoneutron sources in some situations is the presence of high-energy photons with the neutrons. In situations requiring the measurement of a photon signature this extraneous signal can degrade the measurement by reducing the ratio of signal to noise in the measured results, or render some parts of neutron response signatures unusable. Further, the intense photon radiation fields present in pulsed x-ray systems may temporarily disable, or blind, measurement equipment including gas-filled counters, scintillators, and photomultiplier tubes.

\section{Industrial Neutron Producing Accelerators}

Accelerators that produce neutrons come in many different sizes and shapes. In laboratories and research environments accelerator systems often serve multiple purposes, generating neutrons but also generating ion beams (or $\mathrm{x}$ rays) for other research needs as well. In industrial settings though accelerator systems are often purpose-built, optimized for neutron generation and assembled only for neutron applications. Considered in a broad context these accelerator systems may be 
logically categorized in three classes: open-vacuum ion systems, sealed-vacuum ion systems, and $\mathrm{x}$-ray systems. A brief review of accelerators in these classes follows below, together with a discussion of other electric-based systems that produce neutrons which are often included with descriptions of accelerator neutron sources.

\subsection{Open-Vacuum Ion Systems}

The most familiar accelerator neutron sources are large electrostatic (CockcroftWalton and van de Graff designs), radiofrequency (RF) linear accelerator (linac) systems, and cyclotrons employing the light ion reactions of Table 2.[50-54] Several different accelerating arrangements have been developed based on these approaches and deployed in commercial systems; several references have been written describing the scientific operating principles of these devices and the engineering challenges involved in the design, construction, and operation. [55-

58] Rather than repeating these details yet again interested readers are encouraged to consult these references for more information about the underlying details of these systems. What we do choose to emphasize, however, is a key characteristic differentiating accelerator neutron source; namely, the nature of how vacuum is maintained in the system.

These systems, of a form commonly found in low-energy physics laboratories, include an ion source to generate charged particles, an extraction and acceleration section to remove ions from the source and accelerate them to the kinetic energies needed for nuclear reactions, and a target into to which the neutrons are directed and where neutrons are produced. These three components are the key parts of both open-vacuum and sealed-vacuum neutron generators. However, as the name implies, systems in the open-vacuum category also include an active vacuum system that vents to the environment. These vacuum systems keep the ion extraction and acceleration system at a low background pressure. Usually fresh operating gas (hydrogen or deuterium) is injected into a system's ion source, which typically operates at pressures in the $\mathcal{O}(1) \mathrm{Pa}(\sim 10 \mathrm{mTorr})$ range or higher.[59] In these systems the operating gas is restricted from the accelerator section by small orifice(s) of the ion extraction arrangement. Gas that does escape from the ion source enters the main body of the accelerator and is pumped away by the vacuum system.

The mean free path of deuterium at different gas pressures is shown in Table 5. Open-vacuum systems are typically employed for neutron production when accelerating energies in the $\mathrm{MeV}$ range are needed; to achieve these energies accelerating cavities of $\mathcal{O}(1) \mathrm{m}$ in length are common. The vacuum 
system components in these systems are chosen to ensure that the gas pressure within the accelerating regions corresponds to mean free paths longer than these lengths. A photograph of an industrial open-vacuum accelerator system for producing neutrons is shown in Fig. 13.

Table 5 The mean free path of molecules in deuterium gas [60]

\begin{tabular}{cc}
\hline $\begin{array}{c}\text { Pressure } \\
{[\mathrm{Pa}]}\end{array}$ & $\begin{array}{c}\text { Mean Free Path } \\
{[\mathrm{cm}]}\end{array}$ \\
\hline 1 atmosphere & $6 \times 10^{-6}$ \\
$100(750 \mathrm{mTorr})$ & 0.005 \\
$0.1(0.750 \mathrm{mTorr})$ & 5 \\
$10^{-4}\left(7.5 \times 10^{-4} \mathrm{mTorr}\right)$ & 5000 \\
$10^{-7}\left(7.5 \times 10^{-7} \mathrm{mTorr}\right)$ & $5 \times 10^{6}$ \\
\hline
\end{tabular}

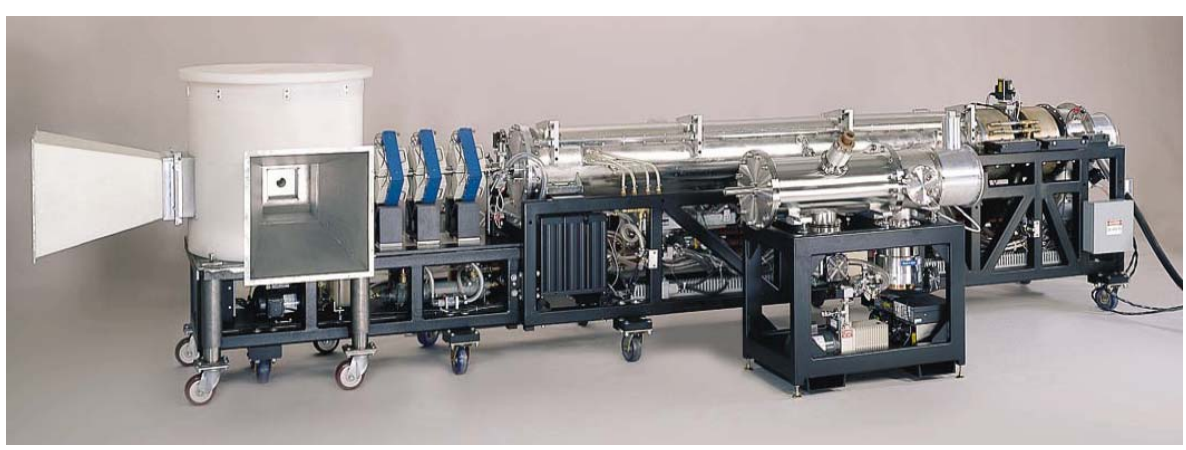

Fig. 13 A photograph of an industrial open-vacuum accelerator system (AssSys Technology, Inc.) with an ion source at the far right side and a shielded neutron production target on the left side.

A strength of open-vacuum systems is their flexibility for service and maintenance. Also, they can be reconfigured for alternate missions through the use of different gas sources or different types of targets. The size of these systems is typically sufficient to support $\mathcal{O}(1) \mathrm{MeV}$ acceleration fields and, due to their relatively large installed footprints and support requirements, it is reasonable to include active coolant circuits on both target heat sinks and in their ion sources. Thus, these systems are also often capable of generating $\mathcal{O}(1) \mathrm{mA}$ ion beam currents. In some situations the size and supporting infrastructure requirements needed for these systems can be a drawback, particularly in fielddeployed situations. Also, vacuum pumping of the interior parts of these 
accelerators, with venting to the environment, essentially precludes the use of tritium in these systems due to modern health physics and environmental regulations.

\subsection{Sealed-Vacuum Ion Systems: Early History}

Open-vacuum accelerators are used in many research laboratories and in some select industrial applications but sealed-vacuum systems are found far more often in current industrial applications. These systems are usually less complicated than their larger open-vacuum cousins and, in their simplest forms, share many technological similarities with light bulbs, vacuum tubes, and switch tubes.[61-63] Sealed-vacuum electronic neutron generators (ENGs) are also generally limited to operating with accelerating voltages less than $<300 \mathrm{kV}$ and to using the DD and DT fusion reactions. However, the neutron yields that can be generated with these systems is substantial for most applications and, for systems operating in the $100-\mathrm{kV}$ range, their small size and low power requirements make them ideal for use in field work.

Sealed-tube neutron generators generally have four main components: i) a vacuum-sealed accelerator tube or 'neutron tube,' ii) a high-voltage power supply to generate the accelerating potential within he neutron tube, iii) a highvoltage accelerator housing, and iv) a control consol to operate the system. Sometimes the neutron tube and high-voltage power supply are collocated within the accelerator housing, sometimes only the neutron tube is within the accelerator housing and the high-voltage power supply is an external assembly connected to the housing and tube using special-purpose high-voltage cables.[64] The accelerator housing helps to protect the neutron tube from external interferences, both physical as well electrical and magnetic. Often it also contains a highvoltage insulating medium such as oil, a liquid fluorocarbon, or sulfur hexafluoride gas to allow the neutron tube to be connected to the high-voltage power supply at voltages higher then could be otherwise sustained in open air.

An example of a commercial vacuum-sealed neutron tube, the core component of electronic neutron generators, is shown in Fig. 14. All neutron tubes contain the main components outlined in the figure including a vacuum envelope (made of a combination of metal, ceramic and/or glass components sealed together), an ion source, an ion acceleration section, and a target. Often they also include an electron suppression device, a metallic structure maintained at an electric field a few $\mathrm{kV}$ more negative than the bias of the target, to deflect electrons liberated from the target as a result of ion bombardment. This prevents these electrons from reaching positive electrodes or the vacuum envelope of the 
tube. Another frequently seen component is a gas reservoir, or getter, comprised of a hydriding metals such as zirconium connected to a heating element such as a thin wire. Deuterium and/or tritium is stored within the reservoir in a solid form when not in use and released into the tube by heating the material during operations. Often, the electrical current used to heat the reservoir is used in a feedback control loop that monitors the ion beam current on the target and adjusted to maintain a constant beam current. One method of configuring the Zetatron neutron tube in Fig. 14 for operation is to apply $+2 \mathrm{kV}$ to the source anode, connect the front and rear cathodes to ground $(0 \mathrm{kV})$, connect $-100 \mathrm{kV}$ to the electron suppressor, and to connect $-99 \mathrm{kV}$ to the target heat sink (alternatively, a resistor or diode may be connected from the electron suppressor to the target, generating the equivalent voltage difference once current (ions) reaches the target). For the cold-cathode (Penning) ion source shown here to operate a magnetic field must be present, an external magnet is often slid over the outside of the ion source to accomplish this but other approaches are also used. Reviewing early design concepts for these devices and acknowledging the adage that form follows function, it is interesting to note that the earliest generation of ENGs, incorporating glass vacuum envelopes, targets, electron suppressors, and support components, were developed with the same general size, shape, and layout of many of today's commercial cold-cathode ion source based neutron tubes.[65-69] 

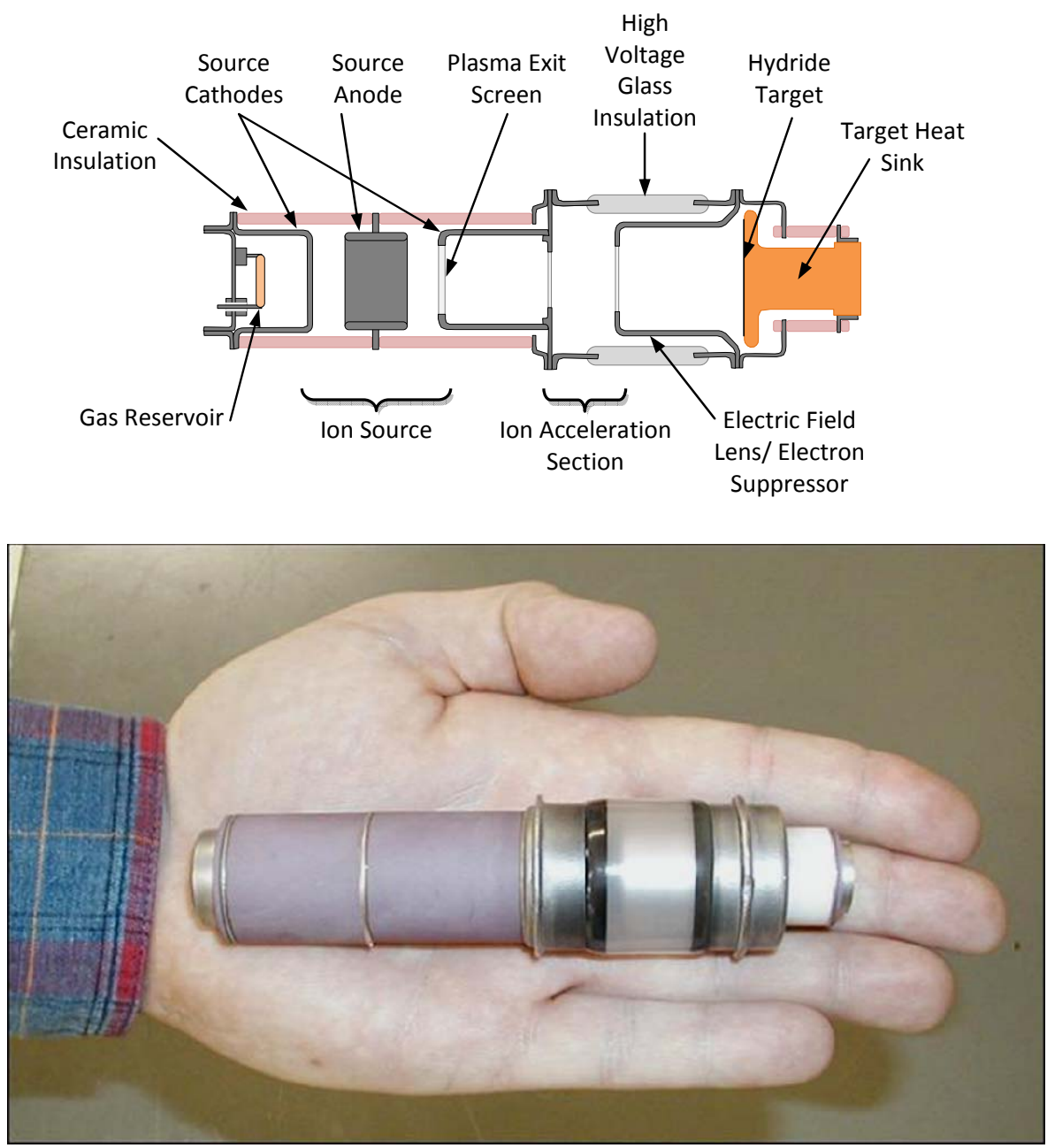

Fig. 14 Schematic cross-section (top) and photo (bottom) of a vacuum-sealed neutron tube, with a cold-cathode ion source, designed by Sandia National Laboratories and sold for commercial applications by Thermo Scientific, Colorado Springs, Colo. (Schematic adapted from reference 70.)

The first patent for the idea of a semi-sealed-tube ENG appears to have been filed in 1938 by Penning.[9] This was followed in 1947 by W. W. Salisbury of the Collins Radio Company of Cedar Rapids, IA.[71] It is unclear, however, if either of these designs was ever manufactured for commercial sale. 
The first patent for the idea of a sealed-tube ENG appears to have been filed by R. E. Fearon and J. M. Thayer of Well Surveys, Inc. of Tulsa, OK in 1949; however, this design did not report on the inclusion of a gas pressure regulator to control the system's operation.[72] Subsequent patents by Schlumberger, Dresser Industries (now Baker Atlas), Well Surveys (later affiliated with Gearhart Industries, now a part of Halliburton), and Mobil Oil Corporation later served to add detail to the well-logging ENG concept.[73-77] Other early ENG designs using vacuum-sealed acceleration tubes to produce neutrons by accelerating deuterium into deuterated and/or tritiated targets, but which weren't specifically intended for use in the oilfield industry, were reported in the same time period by Lawrence Radiation Laboratory and General Electric Research Laboratory, Philips Research Laboratories, Services Electronics Research Laboratory (SERL), and Kaman Corporation in the early 1960s.[78-82] Philips led efforts towards truly commercializing the technology for laboratory purposes and soon thereafter developed laboratory-scale instruments for neutron research.[83,84]

Interest in the technology quickly grew from that point and within the year General Electric reported the development of an ENG to support science for lunar space missions.[85] During the decades that followed many entries were made into the area of commercial ENG manufacturing in the oilfield industry and for use in other areas including the development of products from Efremov (no longer producing ENGs; Soviet Union), Kaman Nuclear (now Thermo Fisher Scientific; USA), Marconi Avionics (no longer producing ENGs, U.K.), Norelco Philips (now SODERN; France), SAMES (no longer producing ENGs; France), SERL (no longer producing ENGs; U.K.), Texas Nuclear (no longer producing ENGs, USA), and VNIIA (All-Russian research Institute of Automatics; Russia).(See reference 86 for examples.) In most cases, however, manufacturers were delineated between either serving the oilfield services market or the otherthan-oilfield market.[87-91] A timeline showing the development and evolution of sealed tube neutron generators is presented in Fig. 15. Today over fifty years of market forces have transformed the commercial sealed-tube ENG market through consolidation, old application market deterioration, new market development, and the expansion of former military ENG manufacturing facilities to civilian international sales. In most cases the DT fusion reaction $\left({ }^{2} \mathrm{H}+{ }^{3} \mathrm{H} \rightarrow\right.$ ${ }^{4} \mathrm{H}+\mathrm{n}$ ) is chosen over the DD reaction since higher energy neutrons are made from DT fusion $(14.1 \mathrm{MeV})$ versus DD fusion $(2.5 \mathrm{MeV})$ and because the maximum achievable yield from a neutron generator using both deuterium and tritium, versus deuterium alone, is $50-100$ times greater depending upon the neutron generator. 


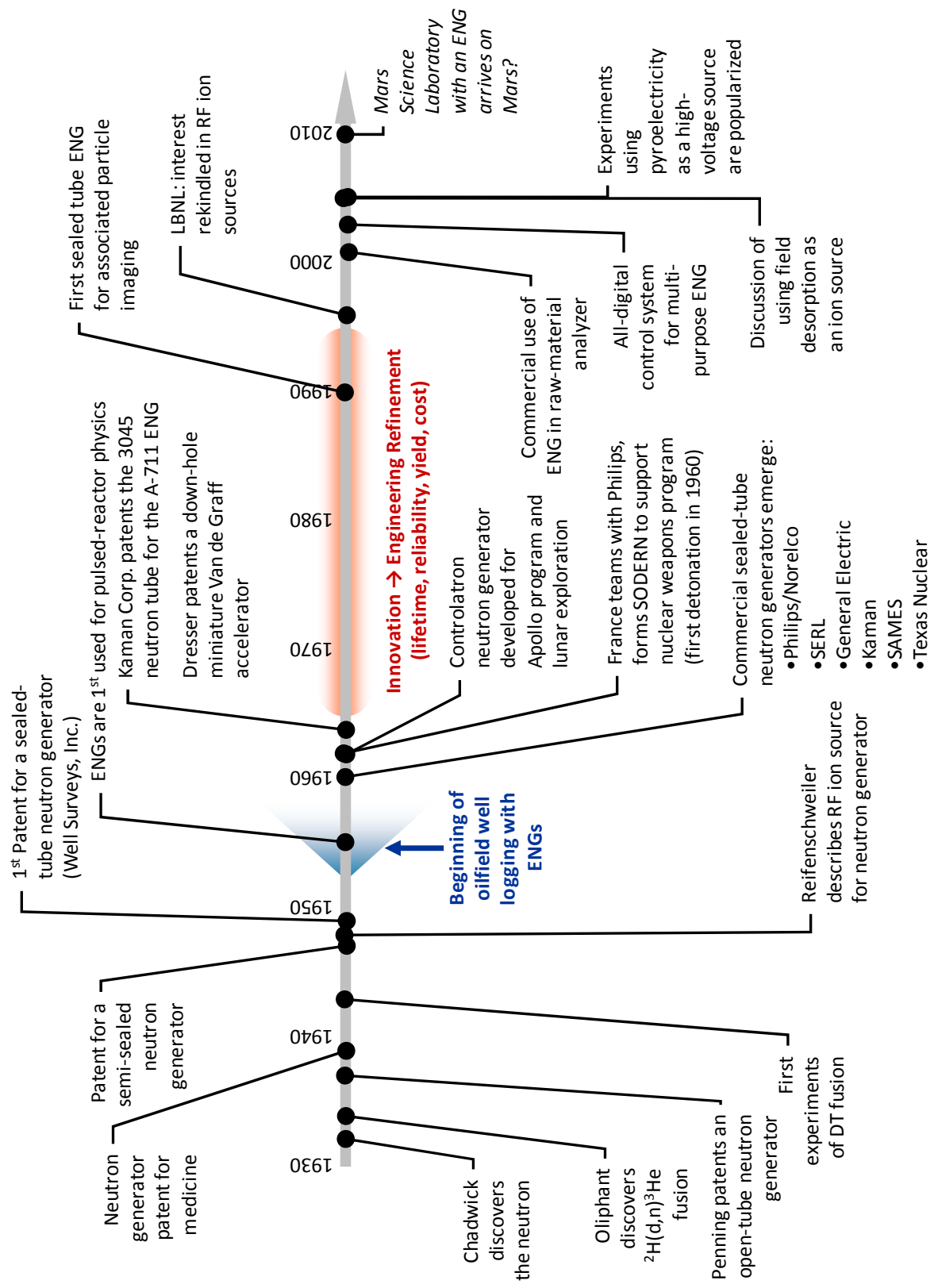

Fig. 15 Event timeline for the development and evolution of sealed tube neutron generators 


\subsection{Industrial ENGs}

Commercial electronic neutron generators may be classified into one of several categories as shown in Table 6. Within this scheme we delineate six different ENG categories including logging-while drilling (LWD), wireline (W), portable $(\mathrm{P})$, general purpose (GP), systems requiring active cooling (AC), and associated particle (AP) ENGs. The LWD-ENGs are the newest class of commercial neutron generators and perhaps the most technically sophisticated. They are designed to operate within the mechanism of a directional oilfield drill and to provide real-time, or near real-time, data on the geological formation in the vicinity of the drill bit. This information is used to help to direct the drilling process and to document the geological characteristics of the drilled hole. Due to the timeliness of this technology and the competitive nature of the oilfield services industry, little public knowledge is available about these devices but some general information can be found from the Schlumberger, Halliburton, and Baker Atlas internet websites. General descriptions of technology related to WENGs is well documented in the literature in both nuclear science journals and journals dealing with wireline data analysis but detailed descriptions remain elusive for the same reasons as with the LWD-ENGs.[92-94] These instruments are highly-engineered devices capable of operating in pulsed modes under extremes of shock and vibration, and at elevated temperatures often exceeding $250{ }^{\circ} \mathrm{C}$. Maximum neutron yields from these devices are found from 1 to $3 \times$ $10^{8}$ neutron per second (DT) and typical operational lifetimes range from 500 to 1500 hours. Both of these parameters have been improved by 3- to 5 -fold over previous generation technology within the last decade. 
Table 6 General categories of commercial electronic neutron generators

\begin{tabular}{|c|c|c|}
\hline Category & Primary Applications & Key Performance Criteria \\
\hline $\begin{array}{l}\text { Logging-While-Drilling } \\
\text { (LWD) }\end{array}$ & $\begin{array}{l}\text { Oilfield drilling } \\
\text { measurements }\end{array}$ & $\begin{array}{l}\text { Extreme ruggedness and small } \\
\text { diameter }\end{array}$ \\
\hline Wireline (W) & Oilfield well surveys & $\begin{array}{l}\text { Extreme ruggedness and small } \\
\text { diameter }\end{array}$ \\
\hline Portable $(\mathrm{P})$ & Security, science & $\begin{array}{l}\text { Light weight }(\sim 10-15 \mathrm{~kg}) \text {, small } \\
\text { overall size, and operational } \\
\text { flexibility }\end{array}$ \\
\hline General Purpose (GP) & $\begin{array}{l}\text { Bulk material analysis, } \\
\text { security, medicine, general } \\
\text { science }\end{array}$ & $\begin{array}{l}\text { Operational flexibility, no active } \\
\text { cooling }\end{array}$ \\
\hline Active Cooling (AC) & $\begin{array}{l}\text { Bulk and trace material } \\
\text { analysis, security, } \\
\text { radiation effects testing, } \\
\text { general science }\end{array}$ & Neutron yield \\
\hline Associated Particle (AP) & Security & $\begin{array}{l}\text { Associated particle detector } \\
\text { performance, beam spot size } \\
\text { (imaging) }\end{array}$ \\
\hline
\end{tabular}

Portable ENGs are another recent addition to the family of ENGs. Technical advancements related to control systems, high-voltage systems, and miniaturization made possible through the use of digital electronics have allowed significant size reductions over previous generation ENGs using analog electronics.[95] P-ENGs are optimized to balance neutronic performance with portability, systems available today weigh approximately $11-12 \mathrm{~kg}$ and can be integrated into battery-powered systems using high-performance Li-ion batteries and small computers with only a marginal weight addition on the order of 5 kg.[64] Development of these systems has been driven by customers in the security field; they are capable of operating over a wide parameter range for pulsing (continuous to $20,000 \mathrm{~Hz}$ ), pulse duty cycle $(0-100 \%)$, and neutron yield (from $0.1 \times 10^{8}$ to $2 \times 10^{8}$ neutrons per second (DT)). However, the compact size and convenience of using these devices, their operational versatility, and the fact that they cost less than the other types of neutron generator have served to expand their use into other application areas as well.

General purpose ENGs, as the name implies, are used in a multitude of application areas including industrial bulk materials analyses, medicine, security, and general science research, by a wide range of users including private companies, hospitals, universities, and national laboratories. The GP-ENG shares operational flexibility in common with the P-ENG but is generally designed to optimize neutronic performance rather than compromising in this area to achieve a lower weight for portability.[96-99] In practice, this optimization for 
performance results in systems with similar pulsing capabilities to portable systems but with maximum neutron yields three to five times higher, approaching $1 \times 10^{9}$ neutrons per second using DT fusion. The $1 \times 10^{9}$ neutrons per second (DT) value is important because it is at this level that a general transition is made in current generation ENG designs from the ability to rely on passive cooling to the need to use active cooling (for the target and often the ion source). Since active cooling requires pumps, heat exchangers and additional control systems, higher yield systems are more complicated and more expensive than the GPENGs.

ENGs requiring active cooling, usually with yields greater than $1 \times 10^{9}$ neutrons per second (DT), are typically 2 times larger than the GP-ENG class of instruments due to their associated heat management systems. AC-ENGs have been developed based on a variety of different ion sources including coldcathodes, hot-cathodes, arc switches, and RF excitation. These higher current ion sources generally require active cooling as well, and the higher beam currents of these instruments lead to the need for larger, higher performance power supplies. For example, nearly all RF-ion source based neutron generators would fall into the AC category due to their the inherently higher beam currents versus coldcathode based ENGs. While it would be possible to build a passively cooled ENG using RF technology it would be impractical to do so for most applications because of the smaller size and lower cost of the above mentioned ENGs, which tend to use either cold-cathode or hot-cathode ion sources. Although the GPENG neutron range is generally bounded at the $1 \times 10^{9}$ level (DT) most ACENGs begin at the $1 \times 10^{10}$ level (DT), probably because of market-driven demands that the increased cost and complexity associated with active cooling should be accompanied significantly higher performance capabilities. Most ACENGs that have been developed in the past have been directed towards radiation effects testing and activation analysis and therefore have not been capable of operating in pulsed modes. Very high yield AC-ENGs have been developed, with neutron yields in some instruments exceeding $1 \times 10^{13}$ neutrons per second for short time periods. In the past it was not uncommon for normal operations of the very highest neutron yield AC-ENGs to release tritium into their local environment.[100,101] However, standards of acceptance regarding the release of radiation have become much more stringent than in the past and today's ACENGs operating in the $10^{10}$ range do not exhibit this behavior.

Interest in neutron generators incorporating detectors to measure the recoil helium atoms associated with the fusion process is not new but has grown over the past decade, primarily in relation to security programs.[102-105] In these systems the associated particle detector, either a scintillator or solid-state 
detector, is used to provide timing information (nanosecond time resolution) about when neutrons are created and directional information regarding their path from the target in the neutron tube. In some cases the directional information provided from the AP detector is used to provide a general indication, or conebeam understanding, of the neutron flight path.[106-110] In other cases AP detectors with high spatial resolution (in systems with small ion beam spots) are used to calculate high-resolution neutron trajectories.[111,112] In the lowresolution form of operation the AP-ENG can be used to improve the signal-tobackground ratio in a measured signature; for example, prompt gamma rays from a region of interest can be discriminated from other gamma rays originating behind the ENG. In the high-resolution form of operation AP-ENGs can be used to develop 2-D and 3-D images using gamma ray or neutron signatures from

objects and topographic data reconstruction tools. AP-ENGs operate in a continuous mode during AP data acquisition although some device shave been built to operate in pulse modes too. Typical neutron yields from these devices are in the $10^{7}$ to $10^{8}$ neutron per second range (DT). In low-resolution systems the beam spots in AP-ENGs may be on the order of $1 \mathrm{~cm}^{2}$ but in high-resolution systems beam spot sizes must be $1-3 \mathrm{~mm}^{2}$.

\subsection{Photoneutron Systems}

Accelerator-based photoneutron sources have not seen wide scale industrial application but have been proposed for use in a small number of applications.[113-116] Moderate energy 5-10 MeV electron linacs and smallscale betatrons are the most commonly proposed systems for use in these applications yet higher energy electron sources clearly have potential as well. The intense photon radiation accompanying neutron production in higher-energy systems makes radiation shielding a challenge while also limiting their applicability when radiation measurements must be made but for radiation effects testing these higher energy photoneutron sources serve a valuable role but care must be taken to correctly assess dosimetric quantities.[117] Accelerator-based photoneutron sources have also been proposed for use in industrial-scale nuclear time-of-flight analytical systems.[47,118-121] Beyond these, however, few concrete descriptions exist of industrial-scale accelerator photoneutron systems.

\subsection{Related Systems}

Two other electronic instruments that generate neutrons must be considered in this context - dense plasma focus (DPF) neutron sources and inertial-electrostatic 
confinement (IEC) neutron sources. Strictly speaking neither of these are particle accelerators but they are often considered in discussions dealing with acceleratorbased neutron sources and electronic neutron generators. Although neither has yet found practical application for industrial purposes they posses some unique operating features which may someday change this.

Dense plasma focus neutron sources are pulsed-power instruments that discharge intense electrical currents to produce plasma.[122-129] Deuterium is the most commonly used gas in these systems, with neutron generation originating from deuterium fusion within the plasma. The electrical discharge and plasma formation in these devices is a highly energetic process and historically has resulted in substantial damage to the plasma generating electrodes. Cumulative damage from repetitive pulses leads to erosion of the electrodes, which eventually requires maintenance and refurbishment. In early generation systems low operating lifetimes of from a few pulses, or "shots," to a few hundred pulses was not uncommon; however, more recent advances have lead to systems capable of $\mathcal{O}\left(10^{8}\right)$ shots without maintenance.[129] Neutron yields from DPF sources using the DD fusion reaction have been reported over a wide range from $10^{8}$ to $10^{11}$ neutrons per shot but the operating lifetime of higher yield systems is significantly reduced. Inherently pulsed systems, DPF pulse rates have been reported from less than one shot per second to up to several hundred Hertz. DPF neutron sources require a substantial investment in instrumentation including electrical storage capacitors, high-power switching systems, and occasionally water cooling support. A unique attribute of DPF neutron sources is that they generate very short duration neutron pulses of $\mathcal{O}(10)$ ns. For systems at the shorter end of this range it may be possible to use DPF neutron sources in time-of-flight measurements.

Inertial-electrostatic confinement neutron sources use hollow, spherical electrode grids to generate ion and electron confinement fields; these fields support the creation and sustainment of low-density deuterium plasma.[130-136] Fusion occurs in these devices as a result of the recirculating transit of ionized deuterium through the plasma; this light-ion fusion is a result of the same D-D fusion that occurs in the particle accelerator systems described above. An often cited advantage of the IEC neutron production approach over more common accelerator-based systems is that they do not require a metal hydride target. Because they do not use solid targets which erode with ion bombardment long operating lifetimes of $\mathcal{O}\left(10^{4}\right)$ hours have been postulated for IEC neutron sources. Reported neutron yields from IEC DD fusion devices cover a wide range from $10^{5}$ to $10^{10}$ neutrons per second. While IEC neutron sources are most often operated in a continuous mode, pulsed-IEC sources have also been 
presented.[137-140] IEC neutron sources are most often spherical in geometry but may also be constructed in cylindrical designs, which may present new possibilities in some applications. IEC neutron sources have higher minimal power requirements than ENG systems of comparable neutron yield $(\mathcal{O}(1000) \mathrm{W}$ vs. $\mathcal{O}(100) \mathrm{W}$ for a DT equivalent yield of $10^{9}$ neutrons per second.)

\subsection{A Comment on Long-Lived Deuterium-Deuterium Neutron Generators}

Occasionally laboratory-scale testing with the DD fusion reaction in openvacuum accelerator system is used as a basis for extrapolating performance expectations for using both the DD and DT reactions in sealed-vacuum systems. This can be misleading. First, while the engineering differences between openvacuum and sealed-vacuum systems may seem superficially obvious, there are numerous practical challenges associated with the production of sealed-vacuum systems intended for long term storage and use. Subtle design considerations must be considered in the development of long-lived vacuum systems including special methods for preparing and cleaning parts (to avoid slow outgassing), design techniques to avoid virtual leaks, and bonding methods to withstand high processing temperatures and to withstand harsh conditions of shock and vibration.[61] Second, in addition to generating neutrons, high-yield DD fusion neutrons sources also produce tritium. Indeed, the reaction cross section for ${ }^{2} \mathrm{H}(\mathrm{d}, \mathrm{p})^{3} \mathrm{H}(\mathrm{Q}=4.033 \mathrm{MeV})$ is $94 \%$ of the ${ }^{2} \mathrm{H}(\mathrm{d}, \mathrm{n})^{4} \mathrm{He}$ reaction cross section at $100 \mathrm{kV}$, as shown in Fig. 16. While a sealed system operates to produce neutrons it is simultaneously breeding tritium within the system. This tritium is dilute with respect to the initial deuterium loading but over time may reach non-trivial

levels. For example, in a DD system producing $10^{9}$ neutrons per second approximately $3.4 \times 10^{14}$ tritium atoms $(0.16 \mathrm{mCi})$ will be produced within the target after 1000 hours of operation. Although widely different target conditions exist in different neutron tubes the tritium content might reasonably equate to a $0.1 \%$ tritium contamination in the target which, considering the higher DT fusion yield versus DD fusion, would contribute up to an extra $10 \%$ to a generator's output.[141] Beside changing the expected yield of this neutron generator it would also alter the emitted neutron spectrum, making it harder than otherwise expected. For sealed-vacuum DD neutron sources designed to reach long operating lifetimes tritium accumulation must be considered. 


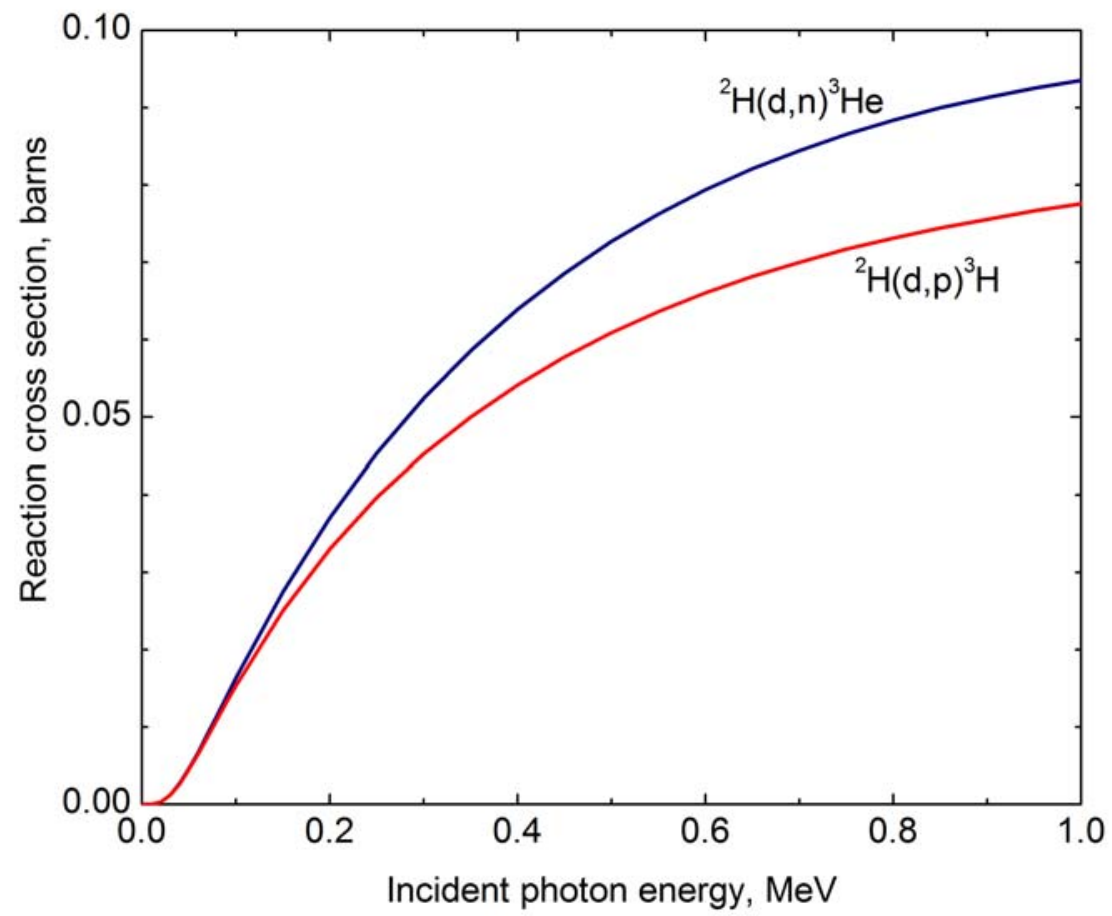

Fig. 16 A comparison of the ${ }^{2} \mathrm{H}(\mathrm{d}, \mathrm{p})^{3} \mathrm{H}$ and ${ }^{2} \mathrm{H}(\mathrm{d}, \mathrm{n})^{4} \mathrm{He}$ reaction cross sections.

\section{Industrial Applications}

Having a high sensitivity to low-Z materials, good penetrability in mid-Z and high- $Z$ materials, and an exceptional sensitivity to isotopic species, neutrons can serve as a versatile and powerful probe radiation for use in nondestructive analyses of materials.[16-19] Within a decade of the neutron's discovery accelerator-based neutron source patents were being filed and system hardware was being developed for medical applications. Following a near complete pause in developments during World War II scientific and commercial interest in the technology quickly resumed and diversified, leading to a rush of advancements in the 1950s and 1960s. During this time the supporting innovations needed to build industrial-grade sealed-vacuum accelerators was perfected, high-yield commercial systems were developed for general-purpose applications, and miniature systems for oilfield applications were first developed.

Evolutionary development was the nature of work in the 1970s through the 1990s with slow but steady improvements in manufacturing techniques and 
commensurate slow but steadily increasing improvements in performance and reliability. Over this period the number of users of high-yield neutron generator systems decreased but the industrial use of electronic neutron generators in oilfield applications continued to grow. Entering the new century, decades of advances in digital electronics finally began to impact accelerator-based neutron generator systems, both open-vacuum and sealed-vacuum systems, with the incorporation of digital systems and computer-based control. Concurrently, more sophisticated scientific modeling and simulation software began to allow a better understanding of the physics occurring within particle accelerators and neutron generators. Building upon all of these advances has lead to the development of better performing and higher efficiency accelerators systems. Applications in sub-surface geophysical exploration remain the single largest area where neutron producing accelerators are used industrially but other notable areas exist as well.[142-147]

\subsection{Neutron Interactions}

The reason for using neutrons in commercial applications is to exploit one or more aspects of how they interact with matter. In some cases the goal is simply to cause a particular interaction in order to observe the effects, for example, in radiation hardness and effects testing of electronics. In most cases where neutrons are used though the goal is to induce a reaction in a test material or sample, and then to observe signatures of the reaction to infer one or more physical properties of the target material. These physical properties include but are not limited to isotopic composition, material thickness, material density, material geometry, and temperature. 
Table 7 Neutron interactions in matter and the measurable signatures of those interactions

\begin{tabular}{|c|c|}
\hline Interactions & Signatures \\
\hline $\begin{array}{c}\text { Elastic } \\
\text { Scattering }\end{array}$ & $\begin{array}{l}\text { - measuring the energy and direction of the recoiling particle (e.g., inside a } \\
\text { detector) to deduce the initial energy and direction of the neutron and, with a } \\
\text { well-characterized neutron source, infer isotopic compositions and distributions } \\
\text { within a sample object } \\
\text { - measuring the energy of the scattered neutron (measuring the time of arrival in } \\
\text { time-of-flight systems), possibly at a one or more selected angles, to infer the } \\
\text { isotopic composition of a sample object, general physical parameters of the } \\
\text { scattering environment; possibly also generating images of the physical } \\
\text { distribution(s) of the scattering isotope(s) } \\
\text { - measuring the transmitted intensity (attenuation) or 2-dimensional (radiography) } \\
\text { transmitted distribution of neutrons to infer nuclei density and distribution } \\
\text { within a sample object; possibly also applying computer tomography techniques } \\
\text { including sample rotation or irradiation from multiple angles to generate 3- } \\
\text { dimensional distribution data (this signature is affected by all of the interaction } \\
\text { mechanisms listed to the left) }\end{array}$ \\
\hline Inelastic & - all of the elastic-scattering signature types \\
\hline Scattering & $\begin{array}{l}\text { - measuring the energy-dependent transmission of neutrons to exploit resonant } \\
\text { characteristics of nuclei for detecting, identifying, and characterizing materials } \\
\text { weasuring the gamma-rays from the decay of excited nuclei, possibly together } \\
\text { with neutron energy information, using this information to infer the isotopic } \\
\text { composition of a sample object and general physical parameters of the } \\
\text { scattering environment; using collimation and/or time-correlation techniques to } \\
\text { infer 2-dimensional or 3-dimensional isotope distributions }\end{array}$ \\
\hline Absorption & $\begin{array}{l}\text { - attenuation, radiography, and computed-tomography as described above } \\
\text { - measuring the energy-dependent transmission of neutrons to exploit resonant } \\
\text { characteristics of nuclei for detecting, identifying, and characterizing materials } \\
\text { - measuring gamma-rays or other particles emitted from nuclei following neutron } \\
\text { absorption, using products from the decay of extremely short-lived compound } \\
\text { nuclei (prompt emissions) or from the decay of radioactive product nuclei } \\
\text { - for fissionable materials, measuring the complex prompt products of nuclear } \\
\text { fission including neutrons, photons, fission products or other charged particles; } \\
\text { also, analyzing the angular distribution of these emissions, analyzing the time- } \\
\text { correlated nature of these emissions including coincidences and higher-order } \\
\text { multiplicity, and/or measuring the } \beta \text {-delayed neutron and photon emissions of } \\
\text { fission products }\end{array}$ \\
\hline
\end{tabular}


The basic reaction mechanisms used to perform these measurements, presented in Table 7, are well understood and include neutron elastic scattering, neutron inelastic scattering, and neutron absorption. Most applications using neutrons seek to measure gamma rays produced from inelastic scattering or absorption (capture), and then to use these gamma rays to infer information about the chemical composition of the volume undergoing interrogation. The gamma rays from these reactions carry a great deal of useful information by themselves, relating to the isotopes they originate from, but can also carry information about the energy of the neutrons leading to their emission when thresholds and resonances are considered. In measurements using pulsed neutron generators, time-correlations between source pulses and gamma-ray detection can also allow inferences about the bulk nature of surrounding media, including density and the average $\mathrm{Z}$ of nearby material. Neutron detection also plays an important role in many applications, particularly involving the detection, identification, or characterization of test objects containing fissionable material. Time-correlations between source neutrons and induced-neutron signatures provide a highly sensitive signature for detecting, identifying, and characterizing these materials. Time-correlations among neutrons from fission when initiated by an external neutron source also provides high-sensitivity signatures for dealing with fissionable materials.

\subsection{Geophysical Exploration}

Early reports of neutron generators for oilfield well logging occurred in the mid1950s by Well Surveys, Inc., of Tulsa, Ok., the McCullough Tool Company of Los Angeles, Calif., and Schlumberger Well Surveying Corp., of Houston, Tex. and Ridgefield, Conn.[72,73,148] Since that time scores of other patents and research papers have been published on improvements and advances in the underlying technology and the oilfield applications that use neutron generators. [93,149-153] Common characteristics of these devices are their small size (in order to fit within small diameter well holes ranging from $5 \mathrm{~cm}$ or less up to 20 $\mathrm{cm}$ ), simplicity of use and low power consumption (in order to allow operation up to several kilometers below the Earth's surface, far from external control systems or power supplies), and extreme ruggedness and durability (to withstand prolonged exposure to high temperatures and pressures, shock, vibration, and corrosive environments). Functionally they generally use the DT fusion reaction, are capable of generating $\mathcal{O}\left(10^{8}\right) \mathrm{n} / \mathrm{s}$, and pulse from $1,000 \mathrm{~Hz}$ to $20,000 \mathrm{~Hz}$ with 
duty cycles of about $10 \%$. A photograph of a typical wireline well-logging tool with an internal electronic neutron generator is shown in Fig. 17.

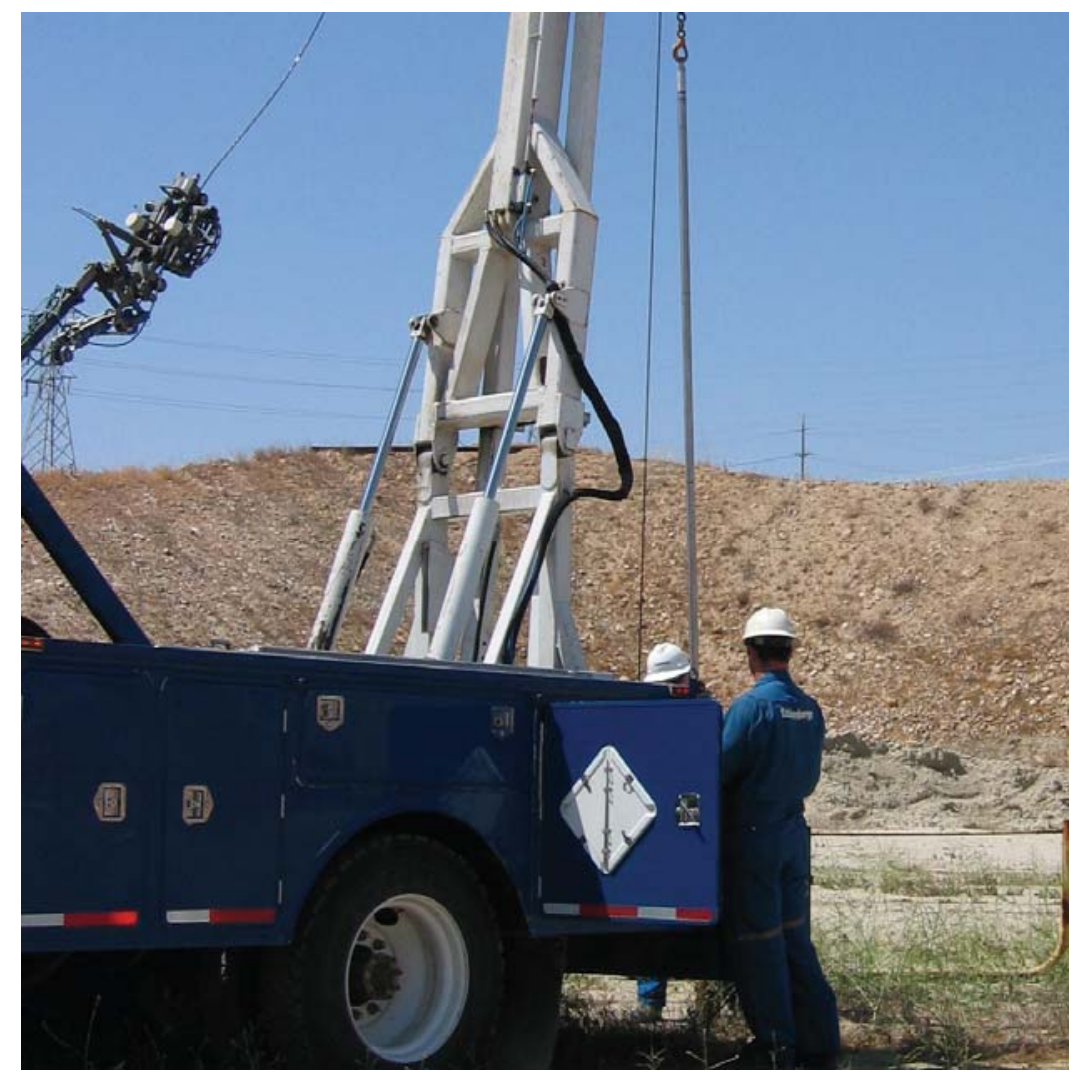

Fig. 17 Photograph of oilfield workers preparing a small-diameter well logging tool (suspended from the crane, behind the truck) with an internal ENG for use in a well. (Courtesy of Jani Reijonen, Schlumberger Ltd.)

In the simplest implementation neutron generators may be used to replace radioisotope neutron sources such as ${ }^{239} \mathrm{PuBe},{ }^{241} \mathrm{AmBe}$, or ${ }^{252} \mathrm{Cf}$, operating in a continuous mode with one or more detectors. (In oilfield applications radioisotope neutron sources are often referred to as chemical neutron sources.) In well-logging measurements the primary challenge is to collect information about the rock near the well without perturbation from neutron generator, well structures, and well fluids. The most common approach for overcoming this challenge is to use multiple detectors at different distances from the neutron generator. The detectors monitor thermal neutron flux intensity 
at different distances above or below the neutron source in the bore hole, usually by detecting gamma rays associated with thermal neutron capture. Detectors close to the generator provide data related to the neutron moderating characteristics near the bore hole while detectors further away provide more information about the bulk properties of the nearby rock, typically out to distances of roughly $30 \mathrm{~cm}$. Ratios are taken of the data from the different detectors to make the analysis more easily interpreted; these types of measurements are referred to as compensated neutron logs. The thermal neutron intensity provides a measure, often qualitative, of moderating characteristics and, more specifically, hydrogen content (water or oil) in the rock. These measurements are often considered a gauge of rock porosity. Using detectors at different spacings, these tools are also good for discriminating the effects of bore-hole casings. A schematic representation of a multiple detector well logging tool, the Schlumberger Accelerator Porosity Sonde, showing the orientation of the ENG and five detectors is shown in Fig. 18.

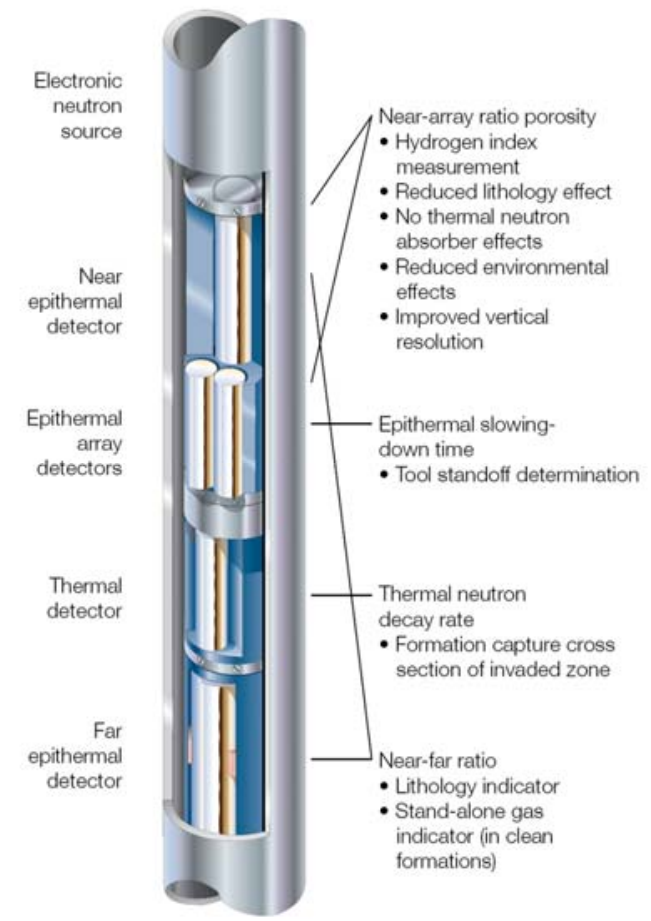

Fig. 18 A schematic representation of a five-detector well logging tool for assessing rock porosity that uses an electronic neutron generator. (Courtesy of Jani Reijonen, Schlumberger Ltd.) 
A second important measurement in neutron well logging is the determination of the rock formation's macroscopic neutron capture cross-section, Sigma, $\Sigma$. This parameter is determined using a pulsed neutron generator with measurements of the decay rate of the thermal flux signature in-between pulses, usually with the use of a photon detector as mentioned above. In combination with other data this can also be used to determine water saturation in the rock for high-salinity environments. Another important pulsed-neutron measurement is the use of inelastic neutron scattering gamma rays from interactions with carbon $\left(\mathrm{E}_{\gamma}=4.43-\mathrm{MeV}\right)$ and oxygen $\left(\mathrm{E}_{\gamma}=6.13 \mathrm{MeV}\right)$. Called the $\mathrm{C} / \mathrm{O} \log$, this measurement takes advantage of a) the reasonably strong reaction probabilities of these two reactions and $b$ ) the large difference in atomic density for carbon and oxygen in water $\left(\right.$ carbon $=0$, oxygen $=3.37 \times 10^{22}$ atoms $\left./ \mathrm{cm}^{3}\right)$ and oil $($ carbon $=$ $3.67 \times 10^{22}$ atoms $/ \mathrm{cm}^{3}$, oxygen $=0$.) Using simple energy windows with a moderate-resolution gamma-ray spectrometer to determine the relative strength of the carbon and oxygen in a measurement, together with other measures of the formation's properties, estimates can be made to discriminate between oil and water in the rock. One novel twist on the $\mathrm{C} / \mathrm{O} \log$ proposed by Chen et al. is the incorporation of an associated particle sensor within a compact sealed-tube neutron generator for well logging applications.[106]

Pulsed electronic neutron generators may also be used in wateractivation tests where water is flowing in the bore hole. In these instruments a gamma-ray detector is placed further away from the generator and it measures the decay of ${ }^{16} \mathrm{~N}$ (7.13-s half-life) produced when high-energy DT neutrons from the generator induce the ${ }^{16} \mathrm{O}(\mathrm{n}, \mathrm{p}){ }^{16} \mathrm{~N}$ reaction in water. This information is of interest in cases where there is a suspicion that water is flowing between the formation and the wall of a bore-hole casing. Yet another neutron logging measurement performed using pulsed ENGs (but also performed using radioisotope neutron sources) is full-fledged gamma-ray spectroscopy using prompt gamma-ray neutron activation analysis (PGNAA). In these measurement a complete gamma-ray energy spectrum is collected and then analyzed using a library of known response functions for each of the major rock elemental constituents expected in the formation $(\mathrm{H}, \mathrm{Si}, \mathrm{S}, \mathrm{Cl}, \mathrm{Ca}, \mathrm{Ti}, \mathrm{Fe}$, etc.) Combining this information with other logging data, estimates may be made of the rock mineralogy in the formation.

Beyond oil and gas research geological applications of accelerator-based neutron generators also exist for the discovery and mapping of minerals and other subsurface materials. One simple example is the use of prompt gamma-ray neutron activation analysis for determining the salinity of water in drill 
holes.[154] Reports have also been made by the company EADS Sodern (Paris, France) for using down-hole PGNAA analysis for locating and mapping copper in bore-hole logging. Other minerals that have been considered for exploration include coal and precious metals. Another notable application for pulsed ENGs in geophysical exploration is uranium logging.[155,156] In these measurements the fission process is exploited by using the ENG to generate short, intense pulses of neutrons and then recording one or more signatures from the fission process including the decay rate of thermal and epithermal neutrons and the intensity of beta-delayed neutrons and gamma rays from the decay of short-lived fission products.

\subsection{Gauging and Radiography}

An early application for which neutron-producing accelerators were developed is gauging and radiography; using fast neutrons as well as thermal neutrons.[157160] Gauging is a one-dimensional measure of attenuation useful for determining material density, fill level in tanks, or through neutron slowing-down phenomena moisture content, as in the oil industry. Both direct attenuation measurements as well as back-scatter measurements detecting neutrons and capture gamma rays have been used with neutrons for gauging applications.[161163] In its application neutron radiography shares many similarities with gauging measurements, with radiography generating 2-dimensional images compared with the single measures of transmission used in gauging. While neutron gauging is a widely used technique for quantifying moisture content in a variety of materials accelerator-based neuron sources are rarely used, probably because of the availability, small size, and low cost of radioisotope neutron sources and because of the relative complexity of accelerator neutron source compared with the overall simplicity of gauging measurements.

In principle neutron radiography is similar to x-ray radiography techniques many people are familiar with. However, for neutron radiography image contrast depends less on bulk density (average-Z) than it does with x-ray imaging; instead it is dependent upon the specific isotopic composition in the transmission area.[164] For example, using fast neutrons low-Z inclusions or contamination from hydrogen are determined much more easily than if $\mathrm{x}$ rays are used. In thermal neutron radiography the familiar thermal-neutron capture agents including boron, gadolinium, and cadmium are most easily imaged and quantified. For fast-neutron radiography neutron-generating accelerators may be used without much infrastructure but for thermal neutron radiography a 
moderating assembly must be used to down-scatter the accelerator's fast neutrons to thermal energies.[165-167]

For radiographic imaging neutrons are most often measured using 2dimensional scintillating screens coupled to charge-coupled device (CCD) imaging cameras, using a bent optic set up to remove the CCD camera from direct exposure to the irradiating neutrons.[168] In high ambient gamma-ray background environments that would damage radiation-sensitive CCD cameras shielding may be used to protect the cameras but an alternate detection technique occasionally employed is the use of neutron-sensitive foils and the activation transfer method.[169,170] In this approach an activation sheet made of a neutron absorbing material that yields an activation product with a half life of $\mathcal{O}\left(10^{4}\right) \mathrm{s}$ is placed behind a neutron-irradiated object. Depending on the composition of the object under test neutrons are either scattered out of the incident neutron beam (e.g., fast neutrons interacting with hydrogen) or absorbed in the object (e.g., slow neutrons absorbed by boron), leaving a negative image of the object's contrast contents on the activation sheet. At the end of exposure the activation sheet is then placed in close contact with a radiography film; as the neutronactivated nuclei in the sheet decay (e.g., copper for fast neutrons or dysprosium for thermal neutrons) they leave an image in the film which can be used for analyses. Storage-phosphor imaging plates coupled with transfer media (to improve their sensitivity to fast neutrons) for use with the activation transfer technique have also been used for neutron radiographic imaging.[170]

Specific examples of industrial neutron radiography using accelerators are numerous and cover many different fields. In general these applications often seek to identify void spaces in man-made materials, seek to detect and quantify damage in structural composite materials, and seek to detect low- $Z$ or high- $Z$ objects among mid-Z materials. [171-175] A few specific examples include the use of fast neutron radiography to inspect composite helicopter rotor blades, the analysis of lithium-aluminum alloy rods, the analysis of voids in castings, and the inspection of air cargo to detect contraband. [176-180] A special perturbation in the radiography field is the use of associated particle neutron generators to provide spatial data for 2-dimensional image reconstruction.[181]

A noteworthy variant of the more familiar transmission radiography is resonant radiography, where image data is collected at multiple neutron energies to exploit resonant absorption effects and provide contrast.[182] One method for conducting these measurements is to use one of the light-ion neutron generating reactions described earlier that produces monoenergetic neutrons at specific angles relative to the ion beam and then to scan an object through different angles reflecting on-resonance and off-resonance energies. Another approach is to use 
time-of-flight techniques with a time-gated detector to generate the on-resonance and off-resonance images.[183] On-resonance transmission images provide contrast to the isotope(s) in the energy ranges analyzed while off-resonance images provide a method of correcting for non-isotope specific neutron scattering and absorption.[184-187] Using high-energy neutrons, commercial measurements have primarily focused on using resonances in carbon, oxygen, and nitrogen for contraband detection including explosives and narcotics, and in the mining industry for the detection and localization of precious minerals. Using slow neutrons generated from a photonuclear neutron source in a TOF arrangement, prototype experiments have also demonstrated the feasibility of using resonant radiography measurements for assaying the isotopic composition of transuranic elements in spent fuel.[118-121]

\subsection{Laboratory Activation Analysis}

Using activation analysis, practical analytical measurements may be made of all of the elements in the periodic table except for helium $\left({ }^{4} \mathrm{He}\right)$; a comprehensive review of these capabilities has been presented by Nargolwalla and Przybylowicz (reference 17). At one time accelerator-based neutron sources found broad application in applied chemistry laboratories as a highly accurate tool for general-purpose chemical assay measurements. [188-193] With the progressive introduction of simpler, and often more accurate, laboratory analytical instruments the use of neutron generators for these applications has diminished but continues in select applications.[194] Electronic neutron generators continue to be used as the primary tool for laboratory analyses in some areas where other competing technical approaches do not provide the same capabilities.[195,196] Of these, the determination of trace quantities of oxygen in metal matrices remains the most important.[197-199]

\subsection{Biomedical Analysis and Applications}

Sealed-tube neutron generators have been used by several investigators as a tool for determining the elemental composition of the human body through PGNAA. In some cases measurements are made to simply quantify the presence of multiple elements in the body directly.[200-202] In other cases, measurements are taken to infer other aspects of body composition, such as the determination of body fat, protein, and water content.[203-208] Beyond human analyses, ENGs have been used for the industrial scanning of livestock to assess lean body mass, using associated particle ENGs in some cases.[209211] For humans body- 
composition studies provide general information on metabolism and health and, more specifically, can be used to examine diseases and other conditions leading to the loss or gain of weight. This information is particularly useful for nutritional studies involving the young and the elderly, and studying the effectiveness of diets focused on countering undesired weight loss. Periodically accelerator-based neutron sources are also considered for use in boron neutron capture therapy (BNCT). [212-216]

\subsection{Bulk Material Analysis}

A quickly growing application area for electronic neutron generators is industrial bulk material analysis (BMA).[217-224] In this application a neutron generator, producing $\mathcal{O}\left(10^{8}\right)$ neutron per second, is used together with a specially engineered moderating structure to generate a mixed-energy neutron spectrum. In most cases this moderating assembly is co-located with a conveyor belt carrying a raw material such as mineral ore, coal, scrap metals, or cement raw materials together with one or more gamma-ray detectors. For high-hydrogenous materials such as coal the source is typically located above the conveyor belt while with lowhydrogenous materials such as metals the source is typically below the conveyor belt, although other options are possible.[221] The choice of where to place the neutron source is dictated by the need to adequately moderate the neutron source while also ensuring the highest possible neutron flux uniformity throughout the measurement volume. In order to afford protection from high radiation fields and to keep interfering signals as low as possible the gamma-ray detectors are usually placed on the opposite side of the conveyor belt from where the ENG(s) is(are) located. A photograph of an industrial system installed at a plant for assaying cement is shown in Fig. 19. In the figure the blue structures contain shielding material to reduce the external radiation fields to the sides of the BMA to acceptable levels while yellow fencing is used to prevent personnel from getting to close to the conveyor belt entrance and exit.

Bulk material assay measurements using neutron generators are most often performed while operating the neutron generator in pulsed mode, collecting fast neutron inelastic scattering data during pulses and thermal neutron PGNAA data in between neutron pulses. The measurement precision of these types of devices is often considered proprietary information but they are generally capable of determining the elemental composition of over a dozen elements in the material carried by the belt to better than a percent relative uncertainty, in rolling averages of a few minutes or less. 


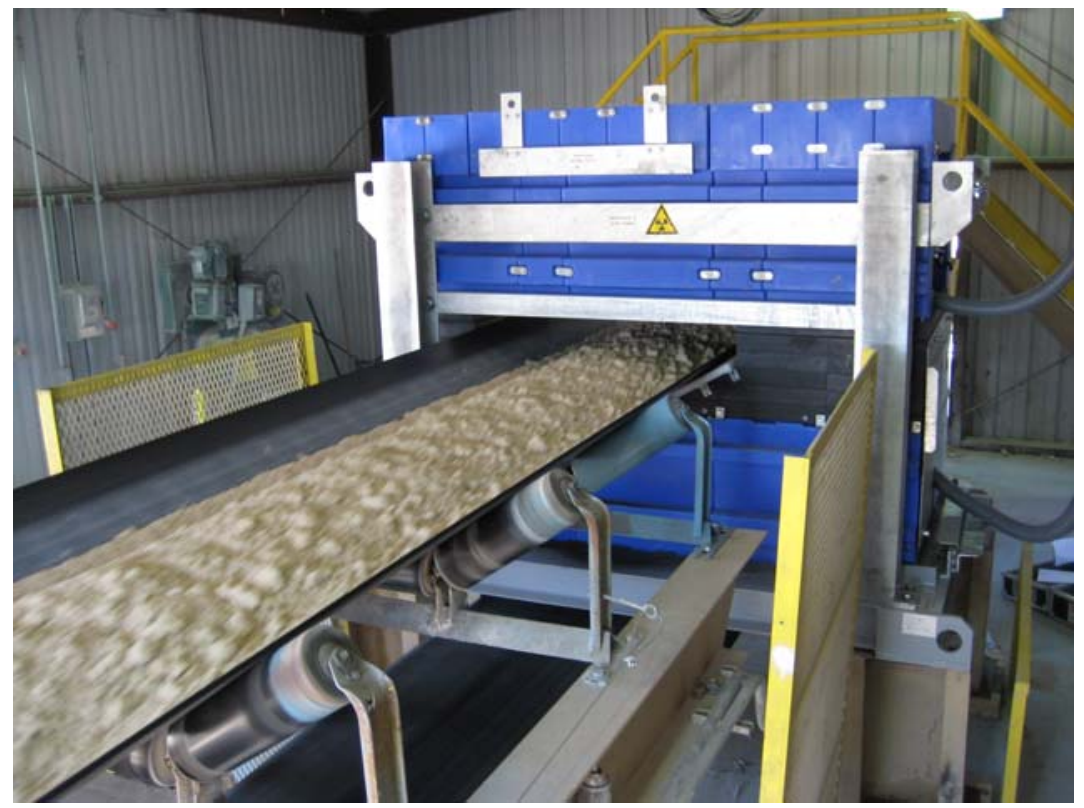

Fig. 19 Photograph of an industrial BMA by EADS SODERN, erected to surround a conveyor belt transporting bulk raw materials. (Photo courtesy of Richard Kahn, EADS SODERN.)

A noteworthy industrial BMA application that has been investigated is the use of fast neutron resonance radiography to image and analyze materials on a conveyor belt. In particular, consideration has been given towards the detection and localization of carbon (diamond) in kimberlite ore. [184,186,225-228] In order to process materials on conveyor belt at a high rate using neutron resonance radiography a very intense neutron source is needed, together with exceptionally capable imaging and data processing resources. A prototype system for this purpose was built for diamond detection in South Africa and, while specific performance data from that system is not available, it is known to have successfully demonstrated the concept at the commercial scale. However, questions regarding the economic viability of this approach and the technology readiness of the underlying systems may still need to be addressed. For very high yield neutron accelerators $\mathcal{O}\left(>10^{12}\right)$ neutrons per second extreme challenges exist in cooling the solid ion beam targets. To address this challenge alternative beam target approaches have been developed including windowless gas targets using high-speed rotating apertures and plasma-window apertures.[229,230] 


\subsection{Radiation Effects Testing}

Accelerator radiation sources are frequently used to test the capability of modern electronic circuitry to withstand ionizing radiation and neutron radiation fields. These tests are of particular importance for electronics used in space, outside of the Earth's atmosphere, where highly energetic solar and cosmic radiation has the potential to deposit significant quantities of energy within the microscopic structures of modern integrated circuits. For neutron susceptible circuitry, however, care must also be taken when using equipment within the Earth's atmosphere, where cosmic-radiation induced spallation reactions in the upper atmosphere can generate $\mathcal{O}(1) \mathrm{GeV}$ neutrons. As a whole, problems in integrated circuits caused by the interaction of single radiation quanta in the circuits are called single event effects (SEE).[231-233] Within the SEE category there are several sub-categories of errors including single event upsets (SEUs), single event latchups (SELs), and single event burnouts (SEBs).

Many resources are dedicated toward studying SEEs to understand their causes and to develop methods to avoid, mitigate, and deal with them. An important aspect of this work is the use of accelerator-based neutron sources to test electronic circuitry and to investigate the causes, and solutions for dealing, with SEEs. Several different neutron-producing reactions and many different accelerator types are used for SEE testing. [234-238] The neutron energies of interest for these tests range from a few $\mathrm{MeV}$ to several hundred $\mathrm{MeV}$. A particular challenge associated with these types of studies is the need to collect accurate dosimetric data in order to fully understand SEE initiation thresholds. Familiar dosimetry tools used in health physics, such as tissue-equivalent sensors, aren't well suited for radiation effects tests and instead silicon-equivalent sensors must be used.

\subsection{Detection of Contraband, High Explosives, and Chemical Weapon Agents}

Since the early 1990's there has been a great deal of work to explore neutron interrogation methods for detecting and identifying contraband and hazardous materials including narcotics, currency, alcohol and liquor, high explosives and unexploded ordinance, and chemical weapon agents. [239-251] The usual idea in these applications is to use a detector to measure inelastic and PGNAA gamma rays resulting from neutron scattering and neutron absorption, seeking first to detect and then often to quantify characteristic gamma rays from elements associated with the contraband or hazardous material. For materials including narcotics, high explosives, and chemical weapon agents, simple generalizations 
for the relative density of a handful of elements can be used to distinguish against benign material. In some cases the presence of one or two key elements alone may be used for detection. For narcotics detection the approach generally seeks to quantify the relative strength of carbon, oxygen, and hydrogen from within an interrogation volume, seeking to match particular ratios for these elements with known ratios for the materials.[252-256] For explosives the presence of nitrogen in high densities is used as a key indicator while full analysis of $\mathrm{N}, \mathrm{C}, \mathrm{O}$, and $\mathrm{H}$ is used to confirm detection and allows for identification in some cases.[257,258] Data for these parameters is presented in Table 8 for some important explosive materials. Demonstrating the small size possible with portable inspection systems, Fig. 20 shows an ENG based system developed by Idaho National Laboratory for detecting and identifying chemical weapon agents and high explosives. Chemical weapon analysis is performed using a similar process to that used with high explosives but with the screening of additional elements including arsenic, chlorine, fluorine, phosphorous, and sulfur.[259,260] 
Table 8 Density and elemental stoichiometry of some common explosive compounds.[282]

\begin{tabular}{|c|c|c|c|c|c|c|c|c|c|c|}
\hline \multirow{2}{*}{$\begin{array}{c}\text { Explosive } \\
\text { Name }\end{array}$} & \multirow{2}{*}{$\begin{array}{c}\text { Molecular } \\
\text { Weight }\end{array}$} & \multicolumn{4}{|c|}{ Stoichiometry } & \multirow{2}{*}{$\begin{array}{r}\text { Density } \\
{\left[\mathrm{g} / \mathrm{cm}^{3}\right]}\end{array}$} & \multicolumn{4}{|c|}{ Elemental Ratios } \\
\hline & & $\mathbf{C}$ & $\mathbf{H}$ & $\mathbf{N}$ & $\mathbf{O}$ & & $\mathrm{C} / \mathrm{O}$ & $\mathbf{H} / \mathbf{N}$ & $\mathbf{C} / \mathbf{N}$ & $\mathbf{O} / \mathbf{N}$ \\
\hline TNT & 227.13 & 7 & 5 & 3 & 6 & 1.65 & 1.17 & 1.67 & 2.33 & 2 \\
\hline $\mathrm{RDX}$ & 222.26 & 3 & 6 & 6 & 6 & 1.83 & 0.5 & 1 & 0.5 & 1 \\
\hline HMX & 296.16 & 4 & 8 & 8 & 8 & 1.96 & 0.5 & 1 & 0.5 & 1 \\
\hline Tetryl & 287.15 & 7 & 5 & 5 & 8 & 1.73 & 0.88 & 1 & 0.88 & 1.6 \\
\hline PETN & 316.20 & 5 & 8 & 4 & 12 & 1.78 & 0.42 & 2 & 1.25 & 3 \\
\hline NG & 227.09 & 3 & 5 & 3 & 9 & 1.59 & 0.33 & 1.67 & 1 & 3 \\
\hline EGDN & 152.10 & 2 & 4 & 2 & 6 & 1.49 & 0.33 & 2 & 1 & 13 \\
\hline AN & 80.05 & - & 4 & 2 & 3 & 1.5 & 0 & 2 & 0 & 1.5 \\
\hline TATP & 222.23 & 9 & 8 & - & 6 & 1.2 & 1.5 & NA & NA & NA \\
\hline DNB & 168.11 & 6 & 4 & 2 & 4 & 1.58 & 1.5 & 2 & 3 & 2 \\
\hline $\begin{array}{l}\text { Picric } \\
\text { Acid }\end{array}$ & 229.12 & 6 & 3 & 3 & 7 & 1.76 & 2 & 1 & 2 & 2.33 \\
\hline
\end{tabular}




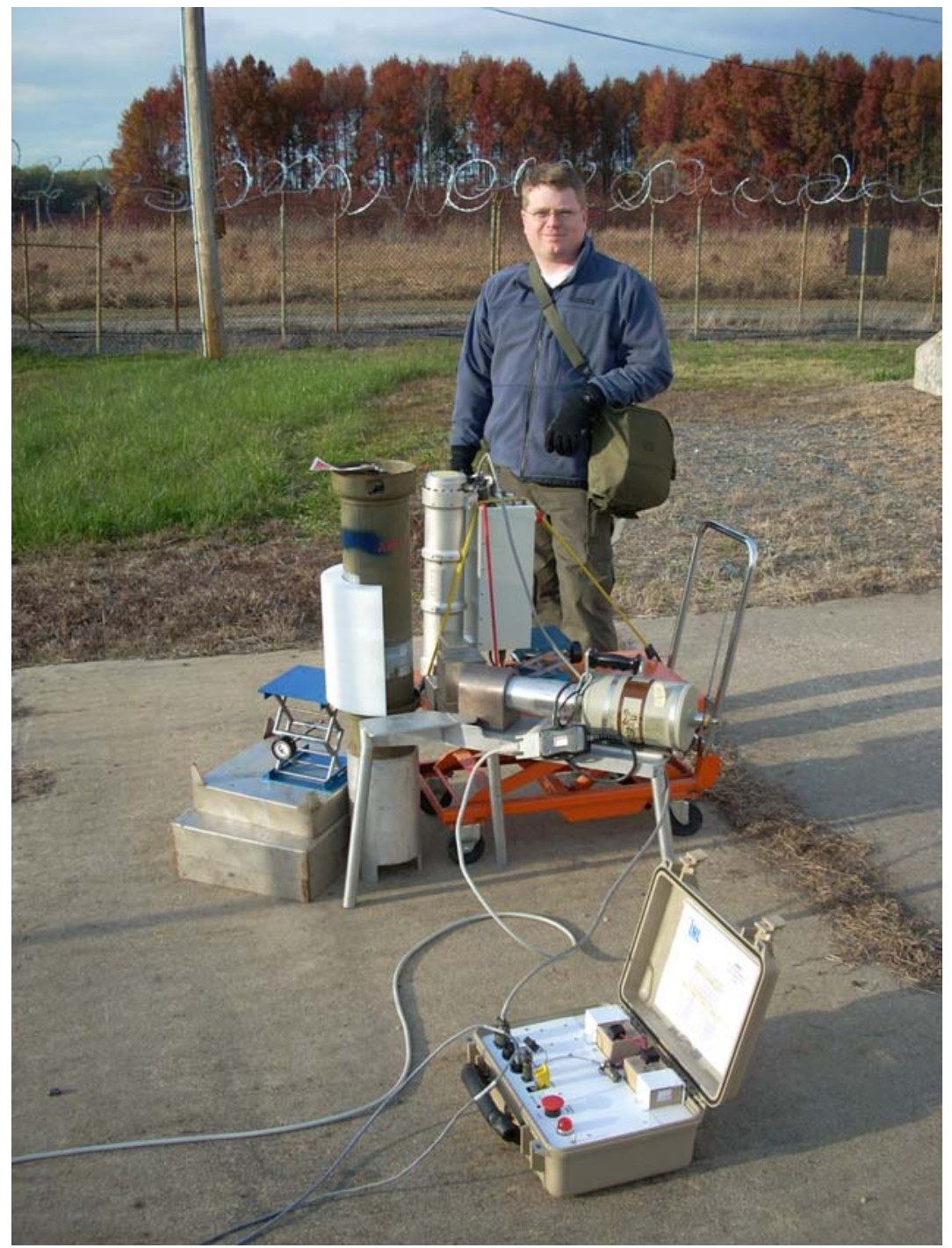

Fig. 20 Photograph of a prototype system for assaying chemical weapons agents and high explosives using a DT ENG (vertical aluminum cylinder in the center), a high purity germanium gamma-ray spectrometer (horizontal, in front of ENG), and a battery and control system (foreground), the only component not shown here is a laptop computer for data analysis. The vertical olive-drab cylinder in the center, to the left of the ENG, is a safety cylinder holding a World War I-era phosgene $\left(\mathrm{COCl}_{2}\right)$ chemical weapon. 
When performing neutron inelastic scattering gamma-ray spectroscopy and PGNAA in the field an ever-present challenge is the presence of background interferences. The key elements used for detecting contraband, $\mathrm{C}, \mathrm{O}$, and hydrogen, are ubiquitous in the natural environment and in legitimate commercial cargo. A standard method for dealing with these legitimate signals in the field is to take a background in the vicinity of the area or object to be screened. This is not always successful though since a representative background environment may not exist; also, in some cases such as cargo screening nearby legitimate cargo clutter may not be easily separated from an interrogation area. To improve the sensitivity of these measurements some detection approaches use the associated particle technique to 'electronically' collimate the inspecting neutron flux to a known region of interest. [261-271] The goal in these cases is to use the spatial information afforded by the AP method to improve the signal to noise ratio for these measurements and to provide a higher level of confidence for the analyses determining contraband in a smaller region of the interrogation volume.

Besides inelastic neutron scattering and neutron absorption gamma-ray spectroscopy several other neutron-based approaches for contraband detection have also been proposed including elastic and inelastic neutron scattering, dualmode photon and neutron radiography, and resonant-neutron transmission radiography.[184-281] Dual-mode radiography provides a quick, informationrich dataset for analyzing aviation cargo for smuggling and customs inspection; for screening aviation luggage and cargo to detect high explosives nuclear resonance radiography has been shown to be a very promising technique. While high explosive screening for aviation security is the single largest application in this category nuclear-based techniques are also being developed for explosive detection in other areas including large-mass explosive detection for vehicleborne explosives and stand-off explosive detection for locating improvised explosive devices.[250-285] Interest also exists in using particle acceleratorbased neutron sources to detect buried landmines.[135,286-288] Small antipersonnel land mines and larger anti-vehicle land mines are an insidious hazard in many parts of the world, with the potential to injure and kill decades after their emplacement. While real-world detection results for these sensors have been inconclusive to date continued work in this area may one day lead to the development of neutron-based inspection systems capable of helping to solve this humanitarian challenge. 


\subsection{Fissionable Material Analysis for Safeguards}

In the realm of nuclear safeguards analysis, measuring and accounting for fissionable material within the nuclear fuel cycle, active neutron interrogation techniques using neutron producing accelerators have been used for several decades.[289-291] In most applications the techniques are based on inducing fission within an interrogation volume and then measuring one or more of the signatures of fission, either prompt or delayed neutrons and gamma rays. In one category of measurements fissionable material is determined within the waste streams of the fuel cycle, mostly at fuel reprocessing facilities.[292-295] For this work assays are made of liquid streams in the reprocessing lines, liquid and solid waste byproducts from different stages of the recycling process, and of solid wastes generated as residual wastes from nuclear material handling. Openvacuum and sealed-vacuum neutron generators are the most commonly used accelerators in these systems but other accelerator types including RFQ accelerators and betatrons have also been used.[296,297] A particularly large number of projects have worked to develop large-scale scanning systems to assay waste drums and waste crates.[298-302] In the well-defined geometry and constraints of these systems fissile material assay detection limits as low as $1 \mathrm{mg}$ have been achieved.[303] In addition to gross material quantification, neutronbased safeguards techniques have also been developed to generate tomographic images of fissionable materials for material protection, control and accountancy.[304]

Pulsed accelerators have also been used for conducting neutron interrogation measurements of nuclear fuel for safeguards measurements, including both fresh and irradiated fuels.[305-308] A wide variety of types of measurements have been used for this work. Traditional fission signature analyses comprise a significant fraction of this work but other approaches, most notably nuclear resonance based techniques, have been tried as well. In the resonant reaction category one approach that has seen increased interest is the use

of the lead slowing down spectrometer (LSDS) technique for assaying plutonium in spent fuel, which exploits the resonant structure of the neutron fission cross sections of actinide elements.[309-314] Similarly, neutron resonance transmission analysis (NRTA) as described earlier has also been proposed as a useful approach for assaying plutonium within spent nuclear fuel. [118-121]. 


\subsection{Fissionable Material Detection for Screening and Security}

Neutron probing techniques have an obvious role in systems for detecting fissionable material for security purposes.[315-319] In most instances developers have approached the field as if ordering from an $A$ La Carte menu of nuclear techniques for particular measurement challenges. Different accelerator types that have been chosen include open-vacuum RFQ machines, many different types of sealed-vacuum electronic neutron generators, some with associated particle imaging, and photoneutron systems. The most commonly used detectors used have been helium-3 based proportional counters, usually moderated with polyethylene and often wrapped in cadmium and/or boron to eliminate sensitivity to room-returning scattered thermal neutrons. Liquid scintillators and capturegated liquid scintillators doped with boron, lithium, or gadolinium have been used for detecting both neutron and gamma rays originating from induced fission. A variety of gamma ray spectrometers including solid-state sensors and inorganic scintillators have been used for this work as well.

One elegant approach for detection, an evolutionary development from radioisotope techniques using AmLi neutron sources, is the use of an accelerator to generate low kinetic energy $60-\mathrm{keV}$ neutrons as a means to induce fission within an interrogation volume.[320] In the approach a liquid scintillator was used to detect prompt fission neutrons during irradiation in recognition of the inherent sensitivity of liquid scintillators to neutrons at the probe source energy. Another frequently applied technique measuring prompt fission neutrons is the neutron die-away technique.[321-328] In this case a pulsed neutron source, usually a DT ENG, is used to irradiate an object while detectors are used to measure neutrons in between ENG pulses. The premise is that fast neutrons entering the system will lose energy and remain resident within the volume for long periods after each pulse, especially in hydrogenous matrices, and that if fissionable material is present fission will occur during this period to generate fast-fission neutrons at time periods much longer than the time needed for neutrons from the original pulse to have scattered to low energies. A key to this approach is to use neutron sensors that are insensitive to thermal neutrons such as shielded ${ }^{3} \mathrm{He}$ detectors or liquid scintillators. An example of the type of data generated from one of these measurements is displayed in Fig. 21. The cumulative neutron count data is shown in a time spectra for the first 3000 microseconds after each pulse, with the data from each pulses added together for a 600-seconds acquisition. During the ENG pulses a plateau is observed, when the ENG output is the dominant neutron source. After each pulse the count intensity quickly drops until the prompt fission die-away signal becomes a 
dominant feature. The die-away signal decays with a pseudo-exponential dependence until background neutrons and neutrons from the decay of $\beta$-delayed neutron-emitting fission products are the only remaining source terms.

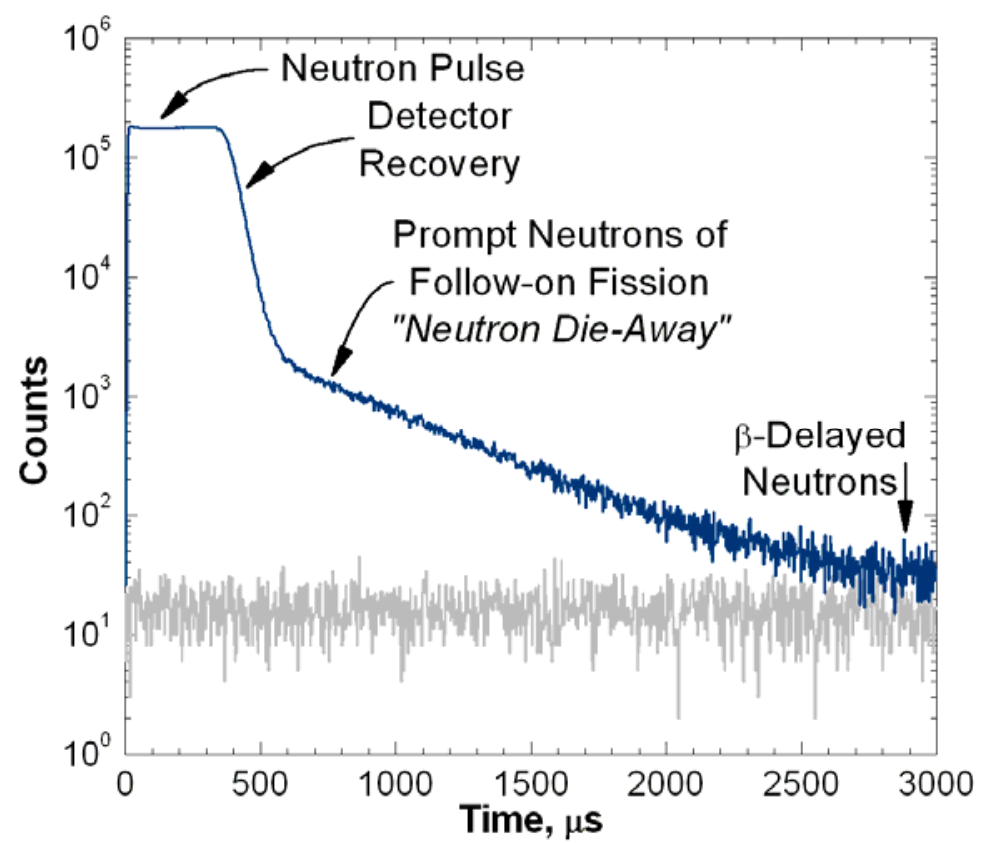

Fig. 21 Neutron die-away data taken of 9.4-kg of highly enriched uranium shielded within a $60 \mathrm{~cm}$ $\times 60 \mathrm{~cm} \times 60 \mathrm{~cm}$ plywood cube using a DT ENG pulsing at $300 \mathrm{~Hz}$ with a $10 \%$ duty cycle, collected using a 10 -atm moderated and shielded ${ }^{3} \mathrm{He}$ proportional counter. Data in blue, background in gray.[329]

Rather than seeking to measure the die-away neutron signature of fission as described above another approach often employed in active neutron interrogation systems is to measure neutrons and gamma rays emitted from the decay of short-live fission products generated within an interrogation volume undergoing irradiation; since nearly all of these fission products decay via $\beta$ particle emission these are sometimes called $\beta$-delayed emissions. Measuring $\beta$ delayed neutrons is advantageous because of the strong signal that can be generated and the low natural background that must be accounted for.[330-334] An analysis of the time dependence of the 200+ fission products that decay with neutron emission yields a six-component exponential decay profile; with half of the total emission probability for uranium having a half-life of $2.23 \mathrm{~s}$ or 
less.[335] In a parallel approach, development has also taken place to seek methods for measuring $\beta$-delayed gamma rays from short-lived fission products and using this as a signature to detect shielded fissionable material.[336-339] While gamma rays continue to be emitted from fission products for long time periods following fission, at short periods a similar time dependence is observed as with the $\beta$-delayed neutrons. An advantage of this approach is that the intensity of the $\beta$-delayed gamma-ray signature is stronger than that of the $\beta$ delayed neutrons. However, data collection can be complicated at very short periods, in between neutron pulses, while thermal neutrons resident in an area are captured and produce multi-MeV capture gamma rays. Both methods can be impacted by the presence of benign interferences when DT neutrons are used, either as a result of the ${ }^{17} \mathrm{O}(\mathrm{n}, \mathrm{p})$ reaction, where ${ }^{17} \mathrm{~N}$ decays with a 4.2-s half life and emits neutrons or the ${ }^{16} \mathrm{O}(\mathrm{n}, \mathrm{p})$ reaction, where ${ }^{16} \mathrm{~N}$ decays with a 7.1 -s half life and emits high-energy gamma rays.[340]

Other signatures from active neutron interrogation that are more challenging to measure and use for detecting shield fissionable material include prompt emissions during high-energy neutron pulses, neutrons from inelastic scattering and associated gamma rays, and neutron-capture gamma rays. One important signature familiar from passive screening is the detection of correlated neutron emissions, neutron coincidence detection and neutron multiplicity analysis. Work in this area has shown the technique effective for both detecting and characterizing fissionable material.[341-343] However, analysis times may be long in some geometries.[344]

\subsection{Other Applications}

Beyond the applications presented above accelerator-based neutron sources have been considered in several other areas too. Sealed-tube electronic neutron generators are the most commonly chosen tool for this work for many reasons including their smaller size, ease of use, portability, and lower cost when compared with larger, open-vacuum systems. Some of these other application areas presented below. Neutron-based analytical techniques can serve a role towards solving many commercial and industrial measurement, gauging, and quality assurance challenges.

- Environmental Analysis ENGs have been used in non-destructive measurements with neutron inelastic scattering activation analysis and PGNAA to detect and quantify hazardous elements in the soil at contaminated locations.[345-347] In the United States an important list 
of hazardous elements is defined by the resource conservation and recovery act (RCRA) guidelines for dealing with hazardous waste, included in this list are the heavy metal elements including $\mathrm{As}, \mathrm{Ba}, \mathrm{Cd}$, $\mathrm{Cr}, \mathrm{Pb}, \mathrm{Hg}, \mathrm{Se}$, and $\mathrm{Ag}$. Neutron-based techniques have also been developed to assay RCRA elements in waste barrels using PGNAA methods.[348-351]

- Soil Analysis Portable ENGs have been used for field measurements to assay carbon content in soil.[352-354] The aim of this work has been to develop a method to quantity carbon present in soil to be able to evaluate long term trends in global climate change, to understand natural carbon concentration variations in soil, and to evaluate man-made methods for carbon sequestration.

- Calibration in Physics Experiments ENGs have been used to generate short-lived calibration standards in modern large-scale physics experiments including Super-Kamiokande, the Sudbury Neutrino Observatory, and the BaBar experiment.[355-358] The radioisotopes produced in these tests, such as ${ }^{16} \mathrm{~N}$ from the ${ }^{16} \mathrm{O}(\mathrm{n}, \mathrm{p})$ reaction or ${ }^{8} \mathrm{Li}$ from the ${ }^{11} \mathrm{~B}(\mathrm{n}, \alpha)$ reaction, decay with the emission of quanta that allow the operators at these experiments to test their equipment under conditions similar to those during regular operations.

- Flow Analysis in Pipes Techniques have been developed to use pulsed accelerator-based neutron sources to analyze the flow of fluids in pipes, including water activation using the ${ }^{16} \mathrm{O}(\mathrm{n}, \mathrm{p})$. In addition to mud and water flow analysis in the wireline industry, as mentioned earlier, analysis techniques have been considered for other industrial process in order to understand flow rates, residence times, and mixing. A comprehensive overview of these techniques has been presented by Perez-Griffo et al.[359,360]

- Extraterrestrial Exploration Beginning with the Apollo program and the development of the Controlatron neutron generator for lunar soil analysis there has been a continued interest in using small ENGs on space probes destined for the Moon and for Mars. [85,361-367] Needing the ability to withstand harsh environments, and requiring very high levels of reliability, vendors of oil-field ENGs have been an important partner in these endeavors though other entities have participated as well. Although no project in this area has yet progressed to full mission deployment current National Aeronautics and Space Administration 
(NASA) programs are in place to send neutron generators to both the Moon and Mars for scientific exploration and discovery.

\section{Summary}

There is a long history of using accelerator-based neutron sources in industrial applications. This chapter has provided a historical overview of the these devices and their evolution. Many different nuclear reactions may be used to generate neutrons in accelerators, using both ion-based reactions as well as photonuclear reactions; in some cases the neutron emission is essentially isotropic but with proper care in choosing the reaction mechanism highly directional and nearly monoenergetic sources may be created. Sealed-vacuum electronic neutron generators are the most-used type of accelerators in industry but other forms, especially RFQ accelerators, have found commercial interest as well. By all accounts the most prevalent use of these devices is in geophysical exploration for the petroleum industry but neutron generating accelerators are found in a diverse array of fields beyond this as well. In measurements the unique nature of the neutron and how it interacts in matter make it an invaluable tool for understanding the physical world while in radiation effects testing accelerator neutron sources provide a realistic method for testing electronic vulnerabilities to cosmic radiation and atmospheric spallation neutrons.

Recognizing the current state of the art in accelerator-based neutron generator technology with an awareness of ongoing and proposed research activities in the public-domain, it is possible to make a reasonable forecast of technological developments that might be expected in the next 5-10 year time period. Such a forecast, however, is necessarily limited in scope from being able to make sound development predictions related to the oilfield services industry due to the closed nature of proprietary developments in that area. Thus, some reasonable expectations in commercial ENG development over this time period, exclusive of the oilfield sector, are given below, in no particular order.

Expansion of ENG Use to Replace Radioisotope Neutron Sources Following general trends observed in the United States an abroad related to security, it is reasonable to expect additional pressure will be placed on radioisotope neutron source users to switch to the use of electronic neutron generators. The use of tritium in ENGs will probably not be a concern in these cases, as the comparative risks of using tritium in radiological dispersal devices versus americium or plutonium is small.[368] 
Very-High-Yield ENGs Using RF Ion Sources On-going research in the USA and South Korea is progressing towards the development of practical high yield ENGs using modern RF ion source technology. High-yield sealed-tube DT ENGs of this type were manufactured by SERL in the 1960's but the technology did not grow from those early efforts. Because of this work it is reasonable to expect that with continued research there will be a reintroduction of commercial ENGs reaching yields of $\mathcal{O}\left(10^{12}\right)$ neutrons per second (DT) with the ability to operate in pulsed modes. The lifetimes of these next-generation systems will likely begin to approach 10,000 hours. These systems will most probably involve active cooling. Motivations for this development will include the need for neutron sources for basic research and education (following the closing of more university research reactors), electronic radiation hardness testing (with further miniaturization of microelectronic circuitry), and security screening.[369,370]

High-Efficiency ENGs Using Field Desorption Ion Sources Current research into the use of microelectromechanical system (MEMS) nanoemitter arrays has shown that quantum field ionization and desorption can be used to generate ions much more efficiently than the plasma-based ion sources used in today's ENGs. If further laboratory successes and research occurs in this field it is realistic to expect it to lead to the development of smaller P-ENGs, in the $5 \mathrm{~kg}$ range, with neutron yields approaching the $10^{9}$ neutrons per second (DT) level without active cooling. Further, the electrical efficiency of these systems should be significantly better than current generation systems, leading to much longer operating periods when powered by batteries. Research in this area will be motivated mostly by security applications. [371-373]

Better Performance AP-ENG Detectors Research is underway to improve the performance of high-resolution scintillator-based particle detectors and solidstate detectors for use in AP-ENGs. In addition, work is also underway to develop better data readout electronics for these systems. Together, if research continues in this area, these expected developments should support the development of next-generation AP-ENG instruments with better performance (lifetime, ruggedness, and beam spot size) than current-generation technology. Research in this area will may be driven nuclear safeguards research, security applications and medical investigations.[374,375] 
Improvements in the User Interface Mirroring developments in the underlying ENG technologies, control system developments will likely be made to improve their ease of use and incorporate more sophisticated self-diagnostics capabilities, building upon the "smart sensor" concept for process instrumentation. For example, rather than setting operating parameters such as high voltage or beam current the user should only have to set a "neutron yield" parameter, letting the instrument determine the optimal combination of voltage and current. Also, technology should allow the ENG to automatically indicate when a component is in need of service, such a replacement of the neutron tube, in order to streamline use in continuous-duty process monitoring applications.

Novel ENG Power Source Development The fact that piezoelectric crystals (exploiting pyroelectric and/or ferroelectric properties) can be used for ENG power sources has been known for decades. However, current small-scale research efforts in this area and other areas may lead to interesting ENG design concepts in the future, perhaps in response to the need to find low-cost long-lived electronic alternatives to radioisotope neutron sources. Regular and ongoing advances in solid state microelectronics may also be expected to yield noteworthy performance improvements over current state of the art compact power systems.[376,377]

Longer-Lived ENG Neutron Tubes The operating lifetime of today's commercial ENGs is primarily limited due to degradation of the hydride target within the sealed neutron tube, which results in a reduction of the overall neutron yield of the ENG and the need to replace the tube. Research can be performed to improve the resistance of hydride targets to this damage mechanism. It is reasonable to be able to expect operational lifetimes exceeding 10,000 hours in the $\mathrm{O}(109)$ neutrons per second (DT) range with relatively stable neutron yields (yield decreasing less than $50 \%$ versus initial yield) through a combination of benefits from improved target design and automated control systems.

Advanced Open-Vacuum Accelerator Concepts Taking a broader view of the future of accelerator-based neutron sources there are conceptual research and design efforts underway for several innovative open-vacuum systems that may have a great impact on future industrial neutron applications. Some of these technology advances include the development of high-efficiency, superconducting cyclotrons, advanced linear accelerators and accelerator storage ring technology, high-gradient accelerating structures, and other exotic ion source and acceleration concepts. 


\section{References}

1 Cockroft, J. D. and Walton, E. T. S., "Experiments with High Velocity Positive Ions, II. The Disintegration of Elements by High Velocity Protons," Proc. Roy. Soc. London, Ser. A. 137 (1932) 229-242.

2 Chadwick, J., "The Existence of a Neutron,” Proc. Roy. Soc. London, Ser. A. 136 (1932) 692708 .

3 Chadwick, J., “Bakerian Lecture, The Neutron,” Proc. Roy. Soc. London, Ser. A. 142 (1933) $1-25$.

4 Oliphant, M. L. E., Harteck, P. and Rutherford, E., "Transmutation Effects Observed with Heavy Hydrogen,” Proc. Roy. Soc. London, Ser. A. 144 (1934) 692-703.

5 Cathcart, B., The Fly in the Cathedral, Penguin Books Ltd., London, England (2004).

6 Oliphant, M. L. E. and Rutherford, E., "Experiments on the Transmutation of Elements by Protons,” Proc. Roy. Soc. London, Ser. A. 141 (1933) 259-281.

7 Oliphant, M. L. E., Kinsey, B. B., and Rutherford, E., "The Transmutation of Lithium by Protons and by Ions of the Heavy Isotope of Hydrogen," Proc. Roy. Soc. London, Ser. A. 141 (1933) 722-733.

8 Zinn, W. H. and Seeley, S., "Production of Neutrons with Low Voltage," Phys. Rev. 50 (1936) 1101.

9 Penning, F. M., "Electronic Device," U.S. Patent 2,211,668 (1940) (The Netherlands, 1937).

10 Schutze, W., "Device for Converting Atoms," U.S. Patent 2,240,914 (1941) (Germany, 1938).

11 Kallmann, H. I., "Method of Producing Neutrons," U.S. Patent 2,251,190 (1941) (Germany 1938).

12 Kallmann, H. I., "Device for the Production of Slow Neutrons," U.S. Patent 2,287,619 (1942) (Germany, 1939).

13 Kallmann, H. I., "Device for Irradiating Objects with Neutrons, Especially for Depicting them by Means of Neutron-Radiation," "U.S. Patent 2,287,620 (1942) (Germany, 1939).

14 Radiation Source Use and Replacement, Abbreviated Version, National Research Council of the National Academies, The National Academies Press, Washington, D.C. (2008).

15 International Approaches to Securing Radioactive Sources Against Terrorism, NATO Science for Peace and Security Series, Wood, W. D. and Robinson, D. M., eds., Springer, Dordrecht, The Netherlands (2009).

16 Marion, J. B. and Fowler, J. L., Fast Neutron Physics, Part I: Techniques \& Part II: Experiments and Theory, John Wiley and Sons, Inc., New York, N.Y. (1963).

17 Nargolwalla, S. S. and Przyblowicz, E. P., Activation Analysis with Neutron Generators, John Wiley and Sons, Inc., New York, N.Y. (1973).

18 Neutron Sources for Basic Physics and Applications, Cierjacks, S., ed., Pergamon Press Ltd., Oxford, England (1983).

19 Csikai, J., CRC Handbook of Fast Neutron Generators, Volume I and Volume II, CRC Press, Inc., Boca Raton, Fla. (1987).

20 Kollár, J., Tatara, M., and Chorvát, D., "Tritium Hazards Connected with the Operation of a Neutron Generator," Int. J. App. Rad. iso. 26 (1975) 635-636.

21 Radiation Protection and Measurement for Low-Voltage Neutron Generators, National Council on Radiation Protection and Measurement, Bethesda, Md. (1983).

22 Krane, K. S., Introductory Nuclear Physics, John Wiley and Sons, Inc., New York, N. Y. (1988).

23 Evaluated Nuclear Data File (ENDF) ENDF/B-VI.8, www.nndc.bnl.gov, U.S. National Nuclear Data Center, Brookhaven National Laboratory (2009). 
24 Shope, L. A., "Theoretical Thick Target Yields for the D-D, D-T, and T-D Nuclear Reactions Using the Metal Occluders Ti and Er and Energies up to $300 \mathrm{keV}$," Report SC-TM-66-247, Sandia national Laboratories, Albuquerque, N.M. (1966).

25 Hawkesworth, M. R., "Neutron Radiography: Equipment and Methods," Atomic Energy Rev. 15 (1977) 169-220.

26 Cecil, F. E. and Nieschmidt, E. B., "Production of $14 \mathrm{MeV}$ Neutrons From D-D Neutron Generators," Nucl. Inst. Meth. Phys. Res. B 16 (1968) 88-90.

27 Kollár, J., Tatara, M., and Chorvát, D., "Tritium Hazards Connected with the Operation of a Neutron Generator," Int. J. App. Rad. Iso. 26 (1975) 635-636.

28 "Radiation Protection and Measurement for Low-Voltage Neutron Generators," NCRP Report No. 72, National Council on Radiation Protection and Measurements, Betheesda, Md. (1983).

29 Lee, C. L., "The Design of an Intense Accelerator-Based Epithermal Neutron Beam Prototype for BNCT Using Near-Threshold Reactions," Ph.D. Dissertation, Nuclear Engineering Department, Massachusetts Institute of Technology, Cambridge, Mass. (1998).

30 Blackburn, B., "High-Power Target Development for Accelerator-Based Neutron Capture Therapy," Ph.D. Thesis, Massachusetts Institute of Technology, Cambridge, Mass. (2002).

31 Drosg, M., "The ${ }^{1} \mathrm{H}\left({ }^{7} \mathrm{Li}, \mathrm{n}\right){ }^{7} \mathrm{Be}$ Reaction as a Neutron Source in the MeV Range," Report LA8852-MS, Los Alamos National Laboratory, Los Alamos, N.M. (1981).

32 Dave, J. H., Gould, C. R., Wender, S. A., Shafroth, S. M., "The ${ }^{1} \mathrm{H}\left({ }^{7} \mathrm{Li}, \mathrm{n}\right)^{7} \mathrm{Be}$ Reaction as an Intense MeV Neutron Source," Nucl. Inst. Meth. Phys. Res. 200 (1982) 285-290.

33 Drosg, M., "Sources of Fast Monoenergetic Neutrons More Recent Developments," Proc. SPIE 2339 (1995) 145-155.

34 U.S. National Nuclear Data Center, http://www.nndc.bnl.gov/qcalc (2009).

35 Drosg, M., "DROSG-2000: Neutron Source Reactions, Data Files with Computer Codes for 59 Monoenergetic Neutron Source Reactions," Report IAEA-NDS-87, International Atomic Energy Agency, Vienna, Austria (2005).

36 Takayanagi, S., Gale, N. H., Garg, J. B., and Calvert, J. M., "Neutron Threshold Measurements in the Reactions ${ }^{9} \mathrm{Be}(\mathrm{p}, \mathrm{n}){ }^{9} \mathrm{~B},{ }^{10} \mathrm{~B}(\mathrm{p}, \mathrm{n}){ }^{10} \mathrm{C},{ }^{11} \mathrm{~B}(\mathrm{p}, \mathrm{n}){ }^{11} \mathrm{C}$," Nucl. Phys. 28 (1961) 494-502.

37 Din, G. U., Nagarajan, M. A., and Pollard, R., "The Study of ${ }^{10} \mathrm{~B}(\mathrm{~d}, \mathrm{n}){ }^{11} \mathrm{C}$ and ${ }^{11} \mathrm{~B}(\mathrm{~d}, \mathrm{n}){ }^{12} \mathrm{C}$ Reactions," Nucl. Phys. A 93 (1967) 190-200.

38 Drosg, M., "Monoenergetic Neutron Sources Based on Two-Body reactions," Contributions to the International Workshop on Fast Neutron Physics, Dresden, Germany, September 5-7 (2002).

39 Antolak, A., Doyle, B., Morse, D., and Provencio, P., "Ion-Induced Gammas for Photofission Interrogation of HEU," Report SAND2006-0995, Sandia National Laboratories, Albuquerque, N.M. (2006)

40 Goldberg, M., "Method and System for Detecting Substances, Such as Special Nuclear Materials," (See Table I) United States Patent 7,381,962 (2008).

41 Auditore, L., Barná, R. C., De Pasquale, D., Trifiró, A., and Trimarchi, M., "Design of a Photoneutron Source Based on a 5 MeV Electron Linac," Proc. EPAC 2004, Lucerne, Switzerland (2004).

42 Bell, Z. W., Chaklov, V. L., and Golovkov, V. M., "Photoneutron Source Based on a Compact $10 \mathrm{MeV}$ Betatron," Report Y/DW/1730 R1, Oak Ridge Y-12 Plant, Oak Ridge, Tenn. (1998).

43 "Handbook on Photonuclear Data for Applications, Cross Sections and Spectra," Report IAEA-TECDOC-Draft No 3, International Atomic Energy Agency, Vienna, Austria (2000). (www-nds.iaea.org/photonuclear)

44 Macklin, R. L., Perez, R. B., de Saussure, G., and Ingle, R. W., "High Energy Resolution Measurement of the ${ }^{238} \mathrm{U}$ Neutron Capture Yield from $1 \mathrm{keV}$ to $100 \mathrm{keV}$," Report ORNL/TM10666, Oak Ridge National Laboratory, Oak Ridge, Tenn. (1988). 
45 Leal, L. C., "Resonance Analysis and Evaluation of the ${ }^{235} \mathrm{U}$ Neutron Induced Cross Section," Report ORNL/TM-11547, Oak Ridge National Laboratory, Oak Ridge, Tenn. (1990).

46 Overberg, M. E., Moretti, B. E., Slovacek, R. E., and Block, R. C., "Photoneutron Target Development for the RPI Linear Accelerator," Nucl. Inst. Meth. Phys. Res. A 438 (1999) 253264.

47 Gunsig, F., Neutron Resonance Spectroscopy, Diploma Thesis, University of Paris, Paris, France (2005).

48 Klug, J., et al., "Development of a Neutron Time-of-Flight Source at the ELBE Accelerator," J. Phys.: Conf. Ser. 41 (2006) 296-305.

49 Flaska, M., "Compact Fast-Neutron Producing Target for High Resolution Cross Section Measurements," Ph.D. Thesis, Technical University of Delft, Delft, The Netherlands (2006).

50 Zinn, W. H. and Seely, S., "A Neutron Generator Utilizing the Deuteron-Deuteron Reaction," Phys. Rev. 52 (1937) 919-923.

51 Alvarez, L. W., "The Production of Collimated Beams of Monoenergetic Neutrons in the Temperature Range $300^{\circ}-10^{\circ} \mathrm{K}$," Phys. Rev. 54 (1938) 609-617.

52 Baker, C. P. and Bacher, R. F., "Experiments with a Slow Neutron Velocity Spectrometer," Phys. Rev. 59 (1941) 332-348.

53 Manley, J. H., Haworth, L. J., and Luebke, E. A., "Developments in Ion Accelerating Tubes," Rev. Sci. Inst. 12 (1941) 587-590.Thonemann, P. C., Moffatt, J., Roaf, D., and Sanders, J. H., "The Performance of a New Radio-Frequency Ion Source," Proc. Phys. Soc. 61 (1948) 483485.

54 Thonemann, P. C., Moffatt, J., Roaf, D., and Sanders, J. H., "The Performance of a New Radio-Frequency Ion Source," Proc. Phys. Soc. 61 (1948) 483-485.

55 Scharf, W., Particle Accelerators and Their Uses. Parts 1 and 2, Harwood Academic Publishers GmbH, Switzerland (1991).

56 Humphries, S., Principles of Charged Particle Acceleration, John Wiley and Sons, Inc., Hoboken, N.J. (1999).

57 Electrostatic Accelerators: Fundamentals and Applications, Hellborg, R., ed., SpringerVerlag, Berlin, Germany (2005).

58 Wiedemann, H., Particle Accelerator Physics, Volume 1, Springer-Verlag, Berlin, Germany (2003).

59 Handbook of Ion Sources, Wolf, B., ed., CRC Press, Inc., Boca Raton, Fla. (1995).

60 Harris, N. S., Vacuum Technology, Edwards High Vacuum, Crawley, Sussex, England (as cited in reference 86).

61 Faure, C., "Tubes Scellés Générateurs Impulsionnels de Neutrons," Acta Electronica 13 (1970) 317-364.

62 Lindin, G. and Massieux, J., "Sealed Neutron Tubes," Report SAND83-6003, Sandia National Laboratories, Albuquerque, N.M. (1985).

63 Kohl, W. H., "Handbook of Materials and Techniques for Vacuum Devices," American Inst. Phys. Press, Woodbury, N.Y. (1995).

64 Chichester, D. L., Simpson, J. D., and Lemchak, M., "Advanced Compact Accelerator Neutron Generator Technology for Active Neutron Interrogation Field Work,” J. Radioanal. Nucl. Chem. 271 (2007) 629-637.

65 Von Kessar, D. A., "Zertrummerungsversuche an Lithium, Bor und Deuterium," Helv. Phyys. Acta 8 (1935) 601-636.

66 Penning, F. M. and Moubis, J. H. A., "Eine Neutronenrohnre Ohne Pumpvorrichtung," Physica 4 (1937) 1190-1199.

67 Bouwers, A., Heyn, F. A., and Kuntke, A., “A Neutron Generator,” Physica 4 (1937) 153-159.

68 Reifenschweiler, O., "Ionenquellen fur Kernphysikalische Untersuchungen," Electrotechink und Maschinenbaus 74 (1957) 96-103. 
69 Gow, J. D. and Ruby, L., "Simple Pulsed Neutron Source based on Crossed-Field Trapping," Rev. Sci. Inst. 30 (1959) 315-317.

70 Shope, L. A., et al., "The Operation and Life of the Zetatron Neutron Tube in a Borehole Logging Application," Int. J. App. Rad. Iso. 34 (1983) 269-272.

71 Salisbury, W. W., "Method and Apparatus for Producing Neutrons," U.S. Patent 2,489,436 (1949) (filed 1947).

72 Fearon, R. E. and Thayer, J. M., "Method for Neutron Well Logging," U.S. Patent 2,712,081 (1955) (filed 1949).

73 Frey, H. B., "Multiple-Target Sources of Radioactive Radiations and Methods Employing the Same," U.S. Patent 2,769,096 (1956) (filed 1952).

74 Goodman, C., "Method and Apparatus for Renewing Targets," U.S. Patent 2,943,239 (1960) (filed 1954).

75 Goodman, C., "Well Logging," U.S. Patent 2,991,364 (1961) (filed 1954).

76 Goodman, C., "Well Logging Methods and Apparatus," U.S. Patent 2,996,618 (1961) (filed 1954).

77 Frentrop, A. H., "Neutron Generating Discharge Tube," U.S. Patent 3,246,191 (1966) (filed 1961).

78 Reifenschweiler, O., "Neutron Generator,," U.S. Patent 3,124,711 (1964) (filed 1959).

79 Gow, J. D. and Pollock, H. C., "Development of a Compact Evacuated Pulsed Neutron Source,” Rev. Sci. Inst. 31 (1960) 235-240.

80 Reifenschweiler, O., "Neutrons from Small Tubes," Nucleonic 18 (1960) 69-71.

81 Hawkins, P. O. and Sutton, R. W., "Compact Pulsed Generator of Fast neutrons," Rev. Sci. Inst. 31 (1960) 241-248.

82 Carr, B. J., "Self-Rectified Positive Ion Accelerator and Neutron Generator," U.S. Patent 3,393,316 (1966) (filed 1964).

83 Buhler, J. S., "The Norelco Neutron Generator," The Norelco Reporter 8 (1961) 87-90.

84 Reifenschweiler, O., "Sealed-Off Neutron Tube: The Underlying Research Work," Philips Res. Repts. 16 (1961) 401-418.

85 "Development of the Controlatron Neutron Source Tube for Lunar Surface Analysis," Report GEX-332, General Electric Company, Milwaukee, WI (1962).

86 "Manual for Troubleshooting and Upgrading of Neutron Generators," Report IAEATECDOC-913, International Atomic Energy Agency, Vienna, Austria (1996).

87 Frentrop, A. H., "Neutron Generator Including an Ion Source with a Massive Ferromagnetic Probe Electrode and a Permanent Magnet-Electrode," U.S. Patent 3,546,512 (1970) (filed 1967).

88 Givens, W. W., "Neutron Accelerator Tube Having Improved Target Section," U.S. Patent 4,311,912 (1982) (filed 1980).

89 Etheridge, D. R., "Neutron Generator Tube," U.S. Patent 4,996,017 (1991) (filed 1985).

90 Chen, F. K. and Liberman, A. D., "Neutron Generator," U.S. Patent 5,293,410 (1994) (filed 1991).

91 Brainard, J. P. and McCollister, D. R., "Secondary Electron Ion Source Neutron Generator," U.S. Patent 5,745,536 (1998) (filed 1996).

92 Goodman, C., "Method and Apparatus for Renewing Targets," U.S. Patent 2,943,239 (1960) (filed (1954).

93 Smith, R. C., Bush, C. H., and Reichardt, J. W., "Small Accelerators as Neutron Generators for the Borehole Environment," IEEE Trans. Nucl. Sci. 35 (1988) 859-862.

94 Pfutzner, H. G. and Mahdavi, M., "Performance Characteristics of the Schlumberger Sealed Tube Neutron Generator System," Proc. SPIE 2339 (1995) 201-207. 
95 Pfutzner, H. G., Groves, J. L., and Mahdavi, M., "A New Compact D-T Pulsed neutron Generator System Suitable for Non-Destructive Analysis Applications," Conf. Record IEEE Nucl. Sci. Symp. Oct. 30 -0 Nov. 5 (1994) 812-815.

96 Reifenschweiler, O., "Sealed-Off Neutron Tube: The Underlying Research Work," Philips Res. Repts. 16 (1961) 401-418.

97 Bach, P., et al., "Industrial Analysis Possibilities Using Long-Life Sealed-Tube Neutron Generators," J. Radioanal. Nucl. Chem. 168 (1993) 393-401.

98 Pfutzner, H. G., Groves, J. L., and Mahdavi, M., "A New Compact D-T Pulsed Neutron Generator Suitable for Non-destructive Analysis Applications," IEEE Conf. Rec. NSS MIC 2 (1994) 812-815.

99 Aleksandrov, V. D., et al., "Application of Neutron Generators for High Explosives, Toxic Agents and Fissile Material Detection,“ App. Rad. Iso. 63 (2005) 537-543.

100 Leonard, B. E., "Experiment, Theory and Data Analysis of Neutrons, Secondary Gammas and EMP for Pulsed Measurements in the Atmosphere," Proc. Workshop on High Intensity Neutron Generators," Las Vegas, Nev., June 17 (1972) 44-78.

101 Jobst, J. E., Burson, Z. G., and Haywood, F. F., "A Self-Replenishing Tritium Target," Proc. Workshop on High Intensity Neutron Generators," Las Vegas, Nev., June 17 (1972) 159-169.

102 Wood, J. D. L. H., "A Sealed-Off $14 \mathrm{MeV}$ Neutron Source Incorporating A Solid State AlphaParticle Detector," Nucl. Inst. Meth. 21 (1963) 49-52.

103 McFadden, R., Martic, P. W., and White, B. L., "An Associated Particle Time of Flight System Using a Position Sensitive Detector," Nucl. Inst. Meth. 92 (1971) 563-567.

104 Gordon, C. M. and Peters, C. W., "A Fast-Neutron Probe for Tomography and Bulk Analysis," App. Rad. Iso. 41 (1990)1111-1116.

105 "Associated Particle Imaging (API)," Report DOE/NV/11718-223, Special Technologies Laboratory, Santa Barbara, Calif. (1998).

106 Chen, Z., et al., "Sealed Tube Neutron Generator Incorporating an Internal Associated-ALP," U.S. Patent 6,297,507 (2001).

107 Kuznetsov, A. V., et al., "Detection of Buried Explosives Using Portable Neutron Sources with Nanosecond Timing," App. Rad. Iso. 61 (2004) 51-57.

108 Perot, B. et al., "Acquisition of Neutron-Induced Signatures of Chemical Agents," Paper SM/EN-07, Proc. Int. Topical Meeting on Nuclear Research Applications and Utilization of Accelerators, International Atomic Energy Agency, Vienna, Austria, May 4-9 (2009).

109 Vakhtin, D. N., Evsenin, A., Osetrov, O., Yurmanov, P., Kuznetsov, A., "Detection of Explosives and Other Illicit Materials by Nanosecond Neutron Analysis," Paper SM/EN-11, Proc. Int. Topical Meeting on Nuclear Research Applications and Utilization of Accelerators, International Atomic Energy Agency, Vienna, Austria, May 4-9 (2009).

110 Le Tourneur, P., "SULIS: A Portable Neutron Device for Explosive and Chemical Detection," Paper SM/EN-17, Proc. Int. Topical Meeting on Nuclear Research Applications and Utilization of Accelerators, International Atomic Energy Agency, Vienna, Austria, May 4-9 (2009).

111 Chichester, D. L., Lemchak, M., and Simpson, J. D., "The API 120: A Portable Neutron Generator for the Associated particle Technique,” Nucl. Inst. Meth. Phys. Res. B 241 (2005) 753-758.

112 Hausladen, P. A., Neal, J. S., and Mihalczo, J. T., "An Alpha Particle Detector for a Portable Neutron Generator for the Nuclear Materials Identification System (NMIS)," Nucl. Inst. Meth. Phys. Res. B, 241 (2005) 835-838.

113 Deye, J. A. and Young, F. C., "Neutron Production from a 10 MV Medical Linac," Phys. Med. Biol. 22 (1977) 90-94.

114 Morgado, R. E., "A Portable 10-MeV Electron Linac as a Neutron Camera," Contained Within Report LA-11393-C, Los Alamos National Laboratory, Los Alamos, N.M. (1987) 10-33. 
115 Auditore, L., Barnà, De Pasquale, D., Trifirò, Trimarchi, M., "Design of a Photoneutron Source Based on a $5 \mathrm{MeV}$ Electron Linac," Proc. EPAC, Lucerne, Switzerland (2004) 23472349.

116 Bell, Z. W., Chaklov, V. L., and Golovkov, V. M., "Photoneutron Source Based on a Compact $10 \mathrm{MeV}$ Betatron," Report Y/DW-1730 R1, Oak Ridge Y-12 Plant, Oak Ridge, Tenn. (1998).

117 Mukherjee, B., Makowski, D., and Simrock, S., "Dosimetry of High-Energy Electron Linac Produced Photoneutrons and the Bremsstrahlung Gamma-Rays Using TLD-500 and TLD-700 Dosimeter Pairs," Nucl. Inst. Meth. Phys. Res. A 545 (2005) 830-841.

118 Schrack, R. A., Behrens, J. W., Johnson, R. G., and Bowman, C. D., "Resonance Neutron Radiography," in Neutron Radiography, Proceedings of the First World Conference, Barton, J. P. and Von Der Hardt, P., eds., D. Reidel Publishing Co. Dordrecht, Holland (1983) 495502.

119 Bowman, C. D., Schrack, R. A., Behrens, J. W., and Johnson, R. G., "Neutron Resonance Transmission Analysis of Reactor Spent Fuel Assemblies," in Neutron Radiography, Proceedings of the First World Conference, Barton, J. P. and Von Der Hardt, P., eds., D. Reidel Publishing Co. Dordrecht, Holland (1983) 503-512.

120 Schrack, R. A., "NBS Work on Neutron Resonance Radiography," Contained Within Report LA-11393-C, Los Alamos National Laboratory, Los Alamos, N.M. (1987) 100-115.

121 Schrack, R.A., "New Applications of Resonance Neutron Radiography," Radiation Effects, 95 (1986) 1309-1313.

122 Bernstein, M. J., "Millimeter and Nanosecond Resolution of Fast Neutrons from an Intense Plasma Discharge," Rev. Sci. Inst. 40 (1969) 1415-1417.

123 Nardi, V. and Brzosko, J. "Plasma Focus Sources," Contained Within Report LA-11393-C Supplemental, Los Alamos National Laboratory, Los Alamos, N.M. (1987).

124 Krakowski, R. A., Sethian, J. D., and Hagenson, R. L., "The High-Density Z-Pinch as a Pulsed Fusion Neutron Source for Fusion Nuclear Technology and Materials Testing," J. Fusion Energy 8 (1989) 269-286.

125 Zakaullah, M., et al., "Comparative Study of Low Energy Mather-Type Plasma Focus Devices," Plasma Sources Sci. Technol. 4 (1995) 117-124.

126 Rapezzi, L., et al., "Development of a Mobile and Repetitive Plasma Focus," Plasma Sources Sci. Technol. 13 (2004) 272-277.

127 Angeli, E., et al., "Preliminary Results on the Production of Short-Lived Radioisotopes with a Plasma Focus Device," App. Rad. Iso. 63 (2005) 545-551.

128 Schmidt, H., Kubes, P., Sadowski, M. J., and Scholz, M., "Neutron Emission Characteristics of Pinched Dense Magnetized Plasmas," IEEE Trans. Nucl. Sci. 34 (2006) 2363-2367.

129 Krishnan, M., Bures, B., and Thompson, J., "A Fast Pulsed Neutron Source Driven by a Pulsed Current Transformer," Paper SM/EN-P17, Proc. Int. Topical Meeting by Nanosecond Neutron Analysis, International Atomic Energy Agency, Vienna, Austria, May 4-9 (2009).

130 Hirsch, R. L., "Inertial-Electrostatic Confinement of Ionized Fusion Gases," J. App. Phys. 38 (1967) 4522-4534.

131 Rider, T. H., A General Critique of Inertial-Electrostatic Confinement Fusion Systems, S.M. Thesis, Massachusetts Institute of Technology, Cambridge, Mass. (1991).

132 Miley, G. H., et al., "Discharge Characteristics of the Spherical Inertial Electrostatic Confinement (IEC) Device," IEEE Trans. Plasma Sci. 25 (1997) 733-739.

133 Nebel, R. A., et al., "The Los Alamos Intense Neutron Source," Report LA-UR-97-1512, Los Alamos National Laboratory, Los Alamos, N. M. (1997).

134 Wehmeyer, A. L., Radel, R. F., and Kulcinski, G. L., "Optimizing Neutron Production Rates From D-D Fusion in an Inertial Electrostatic Confinement Device," Fusion Sci. Tech. 47 (2005) 1260-1264. 
135 Yoshikawa, K. et al., "Research and Development of a Compact Discharge-Driven D-D Fusion Neutron Source for Explosive Detection," Nucl. Inst. Meth. Phys. Res. B 261 (2007) 299-302.

136 Dietrich, C., Improving Particle Confinement in Inertial Electrostatic Fusion for Spacecraft Fusion Power and Propulsion, Ph.D. Thesis, Massachusetts Institute of Technology, Cambridge, Mass. (2007).

137 Gu, Y. Miley, G. and DelMedico, S., "Pulsed IEC Neutron Generator," Digest of Tech. Papers, Tenth IEEE Int. Pulsed Power Conf., Albuquerque, N. M., July 3-6 (1995) 1500-1505.

138 Yamauchi, K. et al., "Performance of Neutron/Proton Source based on Ion-Source-Assisted Cylindrical Radially Convergent Beam Fusion," IEEJ Trans. Fund. Mat. 126 (2006) 11771182.

139 Radel, R. F., et al., "Detection of Highly Enriched Uranium Using a Pulsed D-D Fusion Source, " Report UWFDM-1318, Fusion Tech. Inst., Univ. Wisconsin, Madison, Wis. (2006).

140 Sorebo, J. H., Kulcinski, G. L., Radel, R. F., and Santarius, J. F., "Special Nuclear Materials Detection Using IEC Fusion Pulsed Neutron Source," Fusion Sci. Tech. 56 (2009) 540-544.

141 Cecil, F.E. and Nieschmidt, E. B., "Production of $14 \mathrm{MeV}$ Neutrons From D-D Neutron Generators," Nucl. Inst. Meth. Phys. Res. B 16 (1986) 88-90.

142 Szabo, J. L. and Boutaine, J. L., "Some Examples of Industrial Uses of Neutron Sources," Rad. Prot. Dos. 70 (1997) 193-196.

143 Hussein, E. M. A., Handbook on Radiation Probing, Gauging, Imaging and Analysis, Volume II: Applications and Design, Kluwer Academic Publishers, Dordrecht, The Netherlands (2003) 250.

144 Walker, J., "Uses of Neutrons in Engineering and Technology," Phys. Tecchnol. 13 (1982) 39248.

145 James, W. D., "14 MeV Fast Neutron Activation Analysis in the Year 2000," J. Radioanal. Nucl. Chem. 243 (2000) 119-123.

146 "Use of Accelerator Based Neutron Sources," Report IAEA-TECDOC-1153, International Atomic Energy Agency, Vienna, Austria (2000).

147 Csikai, J. and Dóczi, R., "Applications of Neutron Generators," Chapter 9 in Handbook of Nuclear Chemistry, Vol. 3, Vértes, A., et al., eds., Kluwer Academic Publishers, Dordrecth, The Netherlands (2003).

148 Clark, J. A., The Chronological History of the Petroleum and Natural Gas Industries, Clark Book Co, Houston, Tex. (1963)

149 Youmans, A. H., Millis, C. W., Hopkinson, E. C., and Bishop, W. D., "The Neutron Lifetime Log," Proc. Symp. Soc. Prof. Well Log Analysts (1964).

150 Ethridge, R., Valentine, J., and Stokes, E., "Neutron Generators for Wireline Applications," Conf. Record IEEE Nucl. Sci. Symp., Oct. 22- 27 (1990) 820-824.

151 Odom, R. C., "Design and Initial Field-Test Results of a New Pulsed-Neutron Logging System for Cased Reservoir Characterization," Proc. Symp. Soc. Prof. Well Log Analysts (2008).

152 Johnson, D. E. and Pile, K. E., Well Logging in Nontechnical Language, 2nd Edition, Penn Well Publishing Co., Tulsa, Ok. (2002).

153 Ellis, D. V. and Singer, J. M., Well Logging for Earth Scientists, 2nd Edition, Springer, Dordrecht, The Netherlands (2007).

154 Borsaru, M., et al., "In Situ Determination of Salinity by PGNAA," App. Rad. Iso. 64 (2006) 630-637.

155 Givens, W. W., Mills, W. R., Dennis, C. L., Caldwell, R. L., "Uranium Assay Logging Using a Pulsed 14-MeV Neutron Source," Proc. Symp. Soc. Prof. Well Log Analysts (1976).

156 Thibideau, F. D., "A Pulsed Neutron Generator for Logging," The Log Analyst 18 (1977). 
157 Kallmann, H. I. and Kuhn, E., "Photographische Platte oder Film zur Untersuchung von Substanzen unter Anwendung langsamer Neutronen," Reichspatentamt Patentschrift Nt. 726,278, Deutsches Reich (1942).

158 Holland, L. and Hawkesworth, "Low Voltage Particle Accelerators for Neutron Generation," Nondestructive Testing 4 (1971) 330-337.

159 Berger, H., "Advances in Neutron Radiographic Techniques and Applications: A method for Nondestructive Testing," App. Rad. Iso. 61 (2004) 437-442.

160 Neutron Imaging and Applications: A Reference for the Imaging Community, Anderson, I. A, McGreevy, R. L., and Bilheux, H. Z., eds., Springer Science+Business Media, LLC., New York, N.Y. (2009).

161 Sowerby, B. D., "Neutron and Gamma-Ray Moisture Assay," U.S. Patent 4,884,288 (filed 1989) (Australia, 1985).

162 Xiang, G., et al., "The Development of Oil-Water Interface Neutron Gauge for Oil Storage Tank," J. Isotopes 18 (2005) 26-28.

163 Schweitzer, J. S. and Lanza, R. C., "Nuclear Techniques for the Inspection of Blast Furnaces," AIP Conf. Proc. 475 (1999) 675-677.

164 Lehmann, E., Frei, G., Nordlund, A., and Dahl, B., "Neutron Radiography with $14 \mathrm{MeV}$ Neutrons from a Neutron Generator," IEEE Trans. Nucl. Sci. 52 (2005) 389-393.

165 Cox, A. J. and Francois, P. E., "The Optimization of a Neutron Howitzer," Nucl. Inst. Meth. 92 (1971) 585-58.

166 Spowart, A. R., "Use of a $10^{11} \mathrm{n} / \mathrm{sec}$ Neutron Generator for Neutron Radiography," Nucl. Inst. Meth. 92 (1971) 613-617.

167 Vasilik, D. G., Murri, R. L., and Fisher, G. P., "Thermal-Neutron Radiography with a SealedTube Neutron Generator and Water Moderator," Nucl. Tech. 14 (1972) 279-283.

168 Bogolubov, E., et al., "CCD Detectors for Fast Neutron Radiography and Tomography with a Cone Beam," Nucl. Inst. Meth. Phys. Res. A 542 (2005) 187-191.

169 Barton, J. P., "Toward Neutron Radiography of Radioactive Objects in Hot Cells," Trans. Amer. Nucl. Soc. 10 (1967) 443.

170 Mikerov, V., Samosyuk, V., and Verushkin, S., "Detectors Based on Imaging Plates for Fast Neutron Radiography," Nucl. Inst. Meth. Phys. Res. A 542 (2005) 192-196.

171 Wood, D. E., "Fast-Neutron Radiography with a Neutron Generator," Trans. Amer. Nucl. Soc. 10 (1967) 443-444.

172 Dance, W. E., Cluzeau, S. and Mast, H.-U., "Integration of an Advanced Sealed-Tube Neutron Generator Into a Mobile Neutron Radiology System and Resulting Performance," Nucl. Inst. Meth. Phys. Res. B 59/57 (1991) 907-910.

173 Cluzeau, S. and Le Tourneur, P., "New Neutron Radiography Facility Using a Sealed Neutron Tube Generator GENIE 46," Proc. Regional Meeting: Nuclear Energy in Central Europe Present and Perspectives, Portoroz, Slovenia, June 13-16 (1993) 576-582.

174 Kim, K. H., Klann, R. T., and Raju, B. B., "Fast Neutron Radiography for Composite Materials Evaluation and Testing," Nucl. Inst. Meth. Phys. Res. A 422 (1999) 929-032.

175 Hollas, C. L. and Ussery, L. E., "Transmission Imaging/Diagnostics Using 14-MeV Neutrons," Report LA-UR-04-0989, Los Alamos National Laboratory, Los Alamos, N.M. (2004).

176 Antal, J. J., et al., "A Neutron Radiography System for Field Use," Report MTL TR 89-52, U.S. Army Materials Technology Laboratory, Watertown, Mass. (1989).

177 Antal, J. J., et al., "The Application of Neutron Radioscopy to Lithium-Aluminum Alloy Target Elements," Report MTL TR 90-18, U.S. Army Materials Technology Laboratory, Watertown, Mass. (1990).

178 Allen, D. A., et al., "Neutron Radiography Using a Transportable Superconducting Cyclotron," Nucl. Inst. Meth. Phys. Res. A 353 (1994) 128-133. 
179 Eberhardt, J. E., et al., "Fast Neutron Radiography Scanner for the Detection of Contraband in Air Cargo Containers," App. Rad. Iso. 63 (2005) 179-188.

180 Eberhardt, J. et al., "Fast-Neutron/Gamma-ray Radiography Scanner for the Detection of Contraband in Air Cargo Containers," Proc. SPIE 6213 (2006) 621303-1.

181 Evans, C. J. and Mutamba, Q. B., "The Use of Associated Particle Timing Based on the D+D Reaction for Imaging a Solid Object," App. Rad. Iso. 56 (2002) 711-716.

182 Watterson, J. I. W. and Ambrosi, R. M., "Some Fundamental Considerations in Resonance Imaging Using Fast Neutrons," Nucl. Inst. Meth. Phys. Res. A 513 (2003) 367-373.

183 Dangendorf, V., et al., "Fast Neutron Resonance Radiography in a Pulsed Neutron Beam," Proc. Seventh World Conf. Neutron Radiography, Rome, Italy, Sept. (2002).

184 Chen, G., "Fast Neutron Resonance Radiography for Elemental Imaging: Theory and Applications," Ph.D. Thesis, Massachusetts Institute of Technology, Cambridge, Mass. (2001).

185 Chen, G. and Lanza, R. C., "Fast Neutron Resonance Radiography for Elemental Imaging: Theory and Applications," IEEE Trans. Nucl. Sci. 49 (2002) 1919-1924.

186 Vartsky, D. "Prospects of Fast-Neutron Resonance Radiography and its Requirements for Instrumentation," Proc. Int. Workshop on Fast Neutron Detectors and Applications, Cape Town, South Africa, April 3-6 (2006).

187 Blackburn, B. W., et al., "Fast Neutron Resonance Radiography for Homeland Security," IEEE Nucl. Sci. Symp. Conference Record (2007) 1016-1020

188 Gijbels, R., Speecke, A., and Hoste, J., "An Oxygen Standard for the Determination of Oxygen in Steel by 14-MeV Neutron Activation Analysis," Anal. Chim. Acta 43 (1968) 183198.

189 Breynat, G., Bocquet, J. P., Garrec, J. P., "The Use of Low-Energy Accelerators as Neutron Generators for Activation Work," Nucl. Inst. Meth. 92 (1971) 499.

190 Reimers, P. and Santner, E., "Some Techncial Problems in Activation Analysis Using a 14 MeV Neutron Generator," Nucl. Inst. Meth. 92 (1971) 577-579.

191 Wainerdi, R. E., Zeisler, R., and Schweikert, W. A., "Activation Analysis Opportunities Using a $5 \cdot 10^{12}$ to $5 \cdot 10^{13} \mathrm{n} / \mathrm{sec} 14 \mathrm{MeV}$ Generator," J. Radioanal. Nucl. Chem. 37 (1977) 307-312.

192 James, W. D. and Akanni, M. S., "Application of On-Line Laboratory Computer Analysis to Fast Neutron Activation Oxygen Determinations," IEEE Trans. Nucl. Sci. 30 (1983) 16101613.

193 Mitchell, J. W., Yegnasubramanian, S., and Shepherd, L., "Nitrogen Distribution in Polymers Determined by Proton Track Counting and $14 \mathrm{MeV}$ Neutron Activation Analysis," J. Radioanal. Nucl. Chem. 112 (1987) 425-433.

194 Lupu, R., Nat, A., and Ene, A., "Determination of Gold in Romanian Auriferous Alluvial Sands and Rocks by $14 \mathrm{MeV}$ Neutron Activation Analysis," Nucl. Int. meth. Phys. Res. B 217 (2004) 123-135.

195 Perry, D. L., et al., "The Use of Prompt Gamma Activation Analysis (PGNAA) for the Analyses and Characterization of Materials: Photochromic Materials," Nucl. Inst. Meth. Phys. Res. B 213 (2004) 527-529.

196 Renne, P. R., et al., "Application of Deuteron-Deuteron (D-D) Fusion Neutron to ${ }^{40} \mathrm{Ar}{ }^{39} \mathrm{Ar}$ Geochronology," App. Rad. Iso. 62 (2005) 25-32.

197 James, W. D., "Fast Neutron Activation Analysis at Texas A\&M University," J. Radioanal. Nucl. Chem. 219 (1997) 187-190.

198 James, W. D. and Fuerst, C. D., "Overcoming Matrix Effects in the $14 \mathrm{MeV}$ Fast Neutron Activation Analysis of Metals," J. Radioanal. Nucl. Chem. 244 (2000) 429-434.

199 ASTM Standard E385, 2007, "Standard Test Method for Oxygen Content Using a 14-MeV Neutron Activation and Direct-Counting Technique," ASTM International, West Conshohocken, Pa., 2007, DOI: 10.1520/E0385-07. 
200 Boddy, K., Holloway, I., and Elliott, A., "Some Practical Advantages of Sealed Tube $14 \mathrm{MeV}$ Neutron Generators for Total Body In Vivo Activation Analysis," Phys. Med. Biol. 19 (1974) 379-381.

201 Williams, E. D., Boddy, K., and Haywood, J. K., "Measurement of Neutron Fluence in Total Body In Vivo Neutron Activation Analysis," Phys. Med. Biol. 22 (1977) 1003-1006.

202 Mao, Y., Ehmann, W. D., and Markesbery, W. R., "Simultaneous Determinations of P and N in Human Tissue by $14 \mathrm{MeV}$ Neutron Activation Analysis," Nucl. Inst. Meth. Phys. Res. B 24/25 (1987) 1003-1005.

203 Kehayias, J. J., et al., "In Vivo Determination of Body Fat by Measuring Total Body Composition," Amer. J. Clin. Nutr. 53 (1991) 1339-1344.

204 Kehayias, J. J. and Zhuang, H., "Use of the Zetatron D-T Neutron Generator for the Simultaneous Measurement of Carbon, Oxygen, and Hydrogen In Vivo in Humans," Nucl. Inst. Meth. Phys. Res. B 79 (1993) 555-559.

205 Stamatelatos, I. E., et al., "In Vivo Assessment of Total Body Protein in Rats by Prompt- $\gamma$ Neutron Activation Analysis," Proc. Int. Conf. App. Nucl. Tech.: Neutrons in Research and Industry, Crete, Greece, June 9-15 (1996).

206 Kehayias, J. J., et al., "Medical Applications of Neutron Inelastic Scattering," Proc. SPIE 3769 (1999) 224-231.

207 Kehayias, J. J., "Use of D-T Produced Fast Neutrons for In Vivo Body Composition Analysis: A Reference Method for Nutritional Assessment in the Elderly," Anal. Bioanal. Chem. 379 (2004) 188-191.

208 Ellis, K. J., "Whole-Body Counting and Neutron Activation Analysis," in Human Body Composition, Heymsfield, S. B., et al., eds., Human Kinetics, Champaign, Ill. (2005).

209 Chung, C. and Chen, Y.-Y., "Determination of Nitrogen in Pork Meat Using In-Vivo Prompt Gamma-Ray Activation Technique," J. Radioanal. Nucl. Chem. 169 (1993).

210 Mitra, S. et al., "Whole Body Measurement of C, N, and O Using $14 \mathrm{MeV}$ Neutrons and The Associated Particle Time-of-Flight Technique," Asia Pacific J. Clin. Nutr. 4 (1995) 187-189.

211 Wolff, J. E., et al., "Measuring Body Composition by Fast Neutron Activation Analysis," Proc. New Zealand Soc. Animal Prod. 56 (1996) 1-4.

212 Yanch, J. C., et al., "Accelerator-Based Epithermal Neutron Beam Design for Neutron Capture Therapy," Med. Phys. 19 (1992) 709-721.

213 Lee, C. L., "The Design of an Intense Accelerator-Based Epithermal Neutron Beam Prototype for BNCT Using Near-Threshold Reactions," Ph.D. Thesis, Massachusetts Institute of Technology, Cambridge, Mass. (2000).

214 Cerullo, N., et al., "An Irradiation Facility for Boron Neutron Capture Therapy Application Based on a Radio Frequency Driven D-T neutron Source and a New Beam Shaping Assembly," Rev. Sci. Inst. 73 (2002) 3614-3618.

215 Reijonen, J., et al., "D-D Neutron Generator Development at LBNL," App. Rad. Iso. 63 (2005) 757-763.

216 Kim, K.-O, Kim, J. K., and Kim, S. Y., "Optimized Therapeutic Neutron Beam for Accelerator-Based BNCT by Analyzing the Neutron Angular Distribution from ${ }^{7} \mathrm{Li}(\mathrm{p}, \mathrm{n})^{7} \mathrm{Be}$ Reaction," App. Rad. Iso. 67 (2009) 1173-1179.

217 Bach, P., et al., "Industrial Analysis Possibilities Using Long-Life Sealed-Tube Neutron Generators," J. Radioanal. Nucl. Chem. 168 (1993) 393-401.

218 Beurton, G., Ledru, B., and LeTourneur, P., "Perspectives for On-Line Analysis of Bauxite by Neutron Irradiation," Proc. SPIE 2339 (1995) 424-430.

219 Lebrun, P., et al., "On-Line Analyses of Bulk Materials Using Pulsed Neutron Interrogation," AIP Conf. Proc. 475 (1999) 695-698.

220 Buffler, A., et al., "Material Classification by Fast Neutron Scattering," Nucl. Inst. Meth. Phys. Res. B 173 (2001) 483-502. 
221 Lim, C. S., "Recent Developments in Neutron-Induced Gamma Activation for On-Line Multielemental Analysis in Industry," J. Radioanal. Nucl. Chem. 262 (2004) 525-532.

222 Jing, S.-W., et al., "Development of Pulse Neutron Coal Analyzer," Rev. Sci. Inst. 76 (2005) 045110 .

223 Lim, C. S. and Abernethy, D. A., "On-Line Coal Analysis Using Fast Neutron-Induced Gamma-Rays," App. Rad. Iso. 63 (2005) 697-704.

224 Sowerby, B. D., "Nuclear Techniques for the On-Line Bulk Analysis of Carbon in Coal-Fired Power Stations," App. Rad. Iso. 67 (2009) 1638-1643.

225 Hamm, R. W., et al., "Characterization of a Variable Energy Deuteron RFQ System for Neutron Production," Proc. LINAC98 Conf., Chicago, Ill., August 23-28 (1998) 1010-1012.

226 Tapper, U. A. S. and Over, G. W., "Appareil de Detection de Neutrons," World Patent WO0029873 (2000) (South Africa, 1998).

227 Ambrosi, R. M., "A Model for the Physics of Image Formation in Fast Neutron Radiography," $\mathrm{Ph} . \mathrm{D}$. Thesis, Universoty of Witwatersrand, Johannesburg, Republic of South Africa (1999).

228 Ambrosi, R. M., et al., "Physics of Image Formation in Accelerator-Based Fast Neutron Radiography," Proc. SPIE 4142 (2000) 331-344.

229 Iverson, E. B., "Windowless Gas Targets for Neutron Production," Ph.D. Thesis, Massachusetts Institute of Technology, Cambridge, Mass. (1998).

230 de Beer, A., et al., "Performance of a Plasma Window for a High Pressure Differentially Pumped Deuterium Gas Target for Mono-Energetic Fast Neutron Production - Preliminary Results," Nucl. Inst. Meth. Phys. Res. B 170 (2000) 259-265.

231 Normand, E., "Single-Event Effects in Avionics," IEEE Trans. Nucl. Sci. 43 (1996) 461-474.

232 Normand, E., "Single Event Upset at Ground Level," IEEE Trans. Nucl Sci. 43 (1996) 27422750.

233 Radiation Effects and Soft Errors in Integrated Circuits and Electronic Devices, Schrimpf, R. D. and Fleetwood, D. M., eds., World Scientific Publishing Co. Pte. Ltd., Singapore (2004).

234 Taber, A. H. and Normand, E."Investigation and Characterization of SEU Effects and Hardening Strategies in Avionics," Report DNA-TR-94-123, Defense Nuclear Agency, Alexandria, Va. (1995).

235 Oberg, D. L., et al., "First Observations of Power MOSFET Burnout with High Energy Neutrons," IEEE Trans. Nucl. Sci. 43 (1996) 2913-2920.

236 Ohlsson, M., et al., "Neutron Single Event Upsets in SRAM-Based FPGAs," Proc. IEEE Rad. Effects Data Workshop, Newport Beach, Calif., Jul 24 (1998) 177-180.

237 Johansson, K., et al., "Energy-Resolved neutron SEU Measurements from 22 to $160 \mathrm{MeV}$," IEEE Trans. Nucl. Sci. 45 (1998) 2519-2525.

238 McMarrr, P. J., et al., "14-MeV Neutron and $\mathrm{Co}^{60}$ Gamma testing of a Power MOSFET Optocoupler," IEEE Trans. Nucl. Sci. 50 (2003) 2030-2037.

239 Hussein, E. M. A., "Detection of Explosive Materials Using Nuclear Radiation: A Critical Review," Proc. Int. Soc. Opt. Eng. 1736 (1992) 130-137.

240 Overley, J. C., et al., "Explosives Detection Through Fast-Neutron Time-of-Flight Attenuation Measurements," Nucl. Inst. Meth. Phys. Res. B 99 (1995) 728-732.

241 Gozani, T., "Novel Applications of Fast Neutron Interrogation Methods," Nucl. Inst. Meth. Phys. Res. A 353 (1994) 635-640.

242 Buffler, A., "Contraband Detection with Fast Neutrons," Rad. Phys. Chem. 71 (2004) 853861.

243 Eberhardt, J. E., "Fast Neutron Radiography Scanner for the Detection of Contraband in Air Cargo Containers," App. Rad. Iso. 63 (2005) 179-188.

244 Aleksandrov, V. D., et al., "Application of Neutron Generators for High Explosives, Toxic Agents and Fissile Material Detection," App. Rad. Iso. 63 (2005) 537-543. 
245 Steward, S. and Forsht, D., "Use of Nuclear Techniques to Determine the Fill of Found Unexploded Ordnance," App. Rad. Iso. 63 (2005) 795-797.

246 Strellis, D. and Gozani, T., "Classifying Threats with a 14-MeV Neutron Interrogation System," App. Rad. Iso. 63 (2005) 799-803.

247 Wilhelmsen, K., et al., "Fast-Neutron Analysis at the Swedish Defence Research Agency," J. Radioanal. Nucl. Chem. 271 (2007) 725-730.

248 Koltick, D., et al., "A Neutron Based Vehicle-Borne Improvised Explosives Device Detection System," Nucl. Inst. Meth. Phys. Res. B 261 (2007) 277-280.

249 Gozani, T. and Strellis, D., "Advances in Neutron Based Bulk Explosive Detection," Nucl. Inst. Meth. Phys. Res. B 261 (2007) 311-315.

250 McConchie, S. M., "Detection of Hazardous Materials in Vehicles Using Neutron Interrogation Techniques," Ph.D. Thesis, Purdue University, West Lafayette, Ind. (2007).

251 Seabury, E. H., et al., "A Comparison of Neutron-Based Non-Destructive Assessment methods for Chemical Warfare Materiel and High Explosives," AIP Conf. Proc. 1099 (2009) 928-931.

252 Miller, T. G., et al., "Contraband Detection in Sealed Containers Using Neutron Transmission," Proc. SPIE 2867 (1996) 215-218

253 Zhang, L. and Lanza, R. C., "CAFNA(R) Coded Aperture Fast Neutron Analysis for Contraband Detection: Preliminary Results," IEEE Nucl. Sci. Symp. Conf. Record, Toronto, Canada, November 8-14 (1998).

254 Womble, P. C., et al., "Neutron-Based Portable Drug Probe," AIP Conf. Proc. 475 (1999) 691694.

255 Dokhale, P. A., Csikai, J., and Oláh, L., "Investigations on Neutron-Induced Prompt Gamma Ray Analysis of Bulk Samples," App. Rad. Iso. 54 (2001) 967-971.

256 Dokhale, P. A., et al., "NELIS-An Illicit Drug Detection System," AIP Conf. Proc. 576 (2001) 1061-1064.

257 Steward, S. and Forsht, D., "Use of Nuclear Techniques to Determine the Fill of Found Unexploded Ordnance," App. Rad. Iso. 63 (2005) 795-797.

258 Strellis, D. and Gozani, T., "Classifying Threats with a 14-MeV Neutron Interrogation System," App. Rad. Iso. 63 (2005) 799-803.

259 Caffrey, A. J., et al., "Chemical Warfare Agent and High Explosive Identification by Spectroscopy of Neuron-Induced Gamma Rays," IEEE Trans. Nucl. Sci. 39 (1992) 14221426.

260 Seabury, E. H., et al., "A Comparison of Neutron-Based Non-Destructive Assessment Methods for Chemical Warfare Materiel and High Explosives," AIP Conf. Proc. 1099 (2009) 928-931.

261 Rhodes, E., et al., "APSTNG: Radiation Interrogation for Verification of Chemical and Nuclear Weapons," IEEE Trans. Nucl. Sci. 39 (1041-1045).

262 Nebbia, G., et al., "Performance of a Tagged Neutron Inspection System (TNIS) Based on Portable Sealed Generators," Nucl. Inst. Meth. Phys. Res. A 533 (475-480.

263 Blagus, S., Sudac, D., and Valković, "Hidden Substances Identification by Detection of Fast Neutron Induced $\gamma$ Rays Using Associated $\alpha$ Particle Technique," Nucl. Inst. Meth. Phys. Res. B 213 (2004) 434-438.

264 Donzella, A., et al., "Simulation of a Tagged neutron Inspection System Prototype," J. Phys. Conf. Ser. 41 (2006) 233-240.

265 Lunardon, M., et al., "A Large Area Scanning System Using $14 \mathrm{MeV}$ Neutron Tagged Beams for Non-Destructive Assays," Nucl. Inst. Meth. B 213 (2004) 544-547.

266 Perot, B., "The EURITRACK Project: Status of a Tagged Neutron Inspection System for Cargo Containers," Proceedings IAEA-TM-29225, International Atomic Energy Agency, Vienna, Austria (2006). 
267 Sim, C. M., et al., "Fast Neutron Interrogation System Development for the Detection of Explosive Materials," Nucl. Inst. Meth. Phys. Res. A 605 (2009) 6-68.

268 Perot, B., et al., "Acquisition of Neutron-Induced Gamma Signatures of Chemical Agents," Proc. Int. Topical Meeting on Nuclear Research Applications and Utilization of Accelerators, International Atomic Energy Agency, Vienna, Austria, May 4-8 (2009) SM/EN-07.

269 Evsenin, A. V., et al., "Detection of Explosives and Other Illicit Materials by Nanosecond Neutron Analysis," Proc. Int. Topical Meeting on Nuclear Research Applications and Utilization of Accelerators, International Atomic Energy Agency, Vienna, Austria, May 4-8 (2009) SM/EN-11.

270 Le Tourneur, P., et al., "ULIS: A Portable Device for Chemical and Explosive Detection," Proc. Int. Topical Meeting on Nuclear Research Applications and Utilization of Accelerators, International Atomic Energy Agency, Vienna, Austria, May 4-8 (2009) SM/EN-17.

271 Belichenko, S. G., "Tagged Neutrons from portable Neutron Generator for Detection of High Explosives and Fissile Materials in Cargo Containers," Proc. Int. Topical Meeting on Nuclear Research Applications and Utilization of Accelerators, International Atomic Energy Agency, Vienna, Austria, May 4-8 (2009) SM/EN-P11.

272 Hussein, E. M. A., Lord, P. M., and Bot, D. L., "An Empirical Fast-Neutron Technique for Detection of Explosive-Like Materials," Nucl. Inst. Meth. Phys. Res. A 299 (1990) 453-457.

273 Sowerby, B. D., et al., "Recent Developments in Fast Neutron Radiography for the Interrogation of Air Cargo Containers," Proc. Int. Topical Meeting on Nuclear Research Applications and Utilization of Accelerators, International Atomic Energy Agency, Vienna, Austria, May 4-8 (2009) SM/EN-01.

274 Franklyn, C. B., "Operation of Variable Energy Radio-Frequency Quadrupole Linear Accelerator Systems to Produce Intense Beams of Neutrons for Interrogation of Cargo to Identify Illicit Material," Proc. Int. Topical Meeting on Nuclear Research Applications and Utilization of Accelerators, International Atomic Energy Agency, Vienna, Austria, May 4-8 (2009) SM/EN-12.

275 Gomberg, H. J., "Method and Apparatus for the Noninvasive Interrogation of Objects," U.S. Patent 4,864,142 (1989) (filed 1988).

276 Gribkov, V. A., et al., "Powerful Nanosecond Single-Shot Technique for Detection of Illicit Materials and Explosives," Proc. Int. Topical Meeting on Nuclear Research Applications and Utilization of Accelerators, International Atomic Energy Agency, Vienna, Austria, May 4-8 (2009) SM/EN-19.

277 The Practicality of Pulsed Fast Neutron Transmission Spectroscopy for Aviation Security, National Research Council, Washington, D. C. (1999).

278 The Practicality of Pulsed Fast Neutron Analysis for Aviation Security, National Research Council, Washington, D. C. (2002).

279 Strellis, D. Gozani, T., and Stevenson, J., "Air Cargo Inspection Using Pulsed Fast Neutron Analysis," Proc. Int. Topical Meeting on Nuclear Research Applications and Utilization of Accelerators, International Atomic Energy Agency, Vienna, Austria, May 4-8 (2009) SM/EN05 .

280 Lanza, R. C., "Novel Techniques for Explosive Detection: Current Prospects and Requirements for Future Development," Proceedings IAEA-TM-29225, International Atomic Energy Agency, Vienna, Austria (2006).

281 Goldberg, M. B., et al., "A Dual-Purpose Ion-Accelerator for Nuclear-Reaction-Based Explosives-and SNM-Detection in Massive Cargo," Proc. Int. Topical Meeting on Nuclear Research Applications and Utilization of Accelerators, International Atomic Energy Agency, Vienna, Austria, May 4-8 (2009) SM/EN-13.

282 Bruschini, C., "Commercial Systems for the Direct Detection of Explosives for Explosive Ordnance Disposal Tasks," Subsurface Sensing Tech. App. 2 (2001) 299-336. 
283 Existing and Potential Standoff Explosives Detection Techniques, The National Academies Press, Washington, D. C. (2004).

284 Reber, E. L., et al., "Idaho Explosives Detection System," Nucl. Inst. Meth. Phys. Res. B 241 (2005) 738-742.

285 Detection and Disposals of Improvised Explosives, Schubert, H. and Kuznetsov, A., eds., Springer, Dordrecht, The Netherlands (2009).

286 Kuznetsov, A. V., et al., "Detection of Buried Explosives Usign Portable Neutron Sources with Nanosecond Timing," App. Rad. Iso. 61 (2004) 51-57.

287 Lunardon, M., et al., "Detection of Landmines by Using $14 \mathrm{MeV}$ Neutron Tagged Beams," App. Rad. Iso. 61 (2004) 43-49.

288 Le Tourneur, P., "Landmine Detection by Associated Particle Imaging Neutron Technique: Lab and Field Results," Proceedings IAEA-TM-29225, International Atomic Energy Agency, Vienna, Austria (2006).

289 Gozani, T., "Physics of Nuclear Materials Safeguards Techniques," Nucl. Tech. 13 (1972) 819.

290 Gozani, T., "Active Nondestructive Assay of Nuclear Materials," Report NUREG/CR-0602, U.S. Nuclear Regulatory Commission, Washington, D. C. (1981).

291 Clapham, M., et al., "Performance Comparison of Different Active Neutron Interrogation Techniques for Safeguards Applications," Proc. 19th ESARDA Symp. on Safeguards and Nuclear Material Management, Montpellier, France, May 13-15 (1997) 359-367.

292 Jover, P., "Equipment for the Detection of Residual Fuel in Leached Hulls by Neutrons Counting," IEEE Trans. Nucl. Sci. 17 (1970) 517-519.

293 McDonald, B. J., Fox, G. H., and Bremner, W. B., "Non-Destructive Measurement of Plutonium and Uranium in Process Wastes and Residues," Report IAEA-SM-201/61, International Atomic Energy Agency, Vienna, Austria (1976).

294 Romeyer-Dherbey, J., Passard, C., and Cloue, J., "Measurement of Fissile Material Concentration in Liquids by Active Neutron Interrogation," Proc. ESARDA 15th Annual Symposium, Rome, Italy, may 11-13 (1993) 543-549.

295 Raoux, A.-C., et al., "Non Destructive Radioactive Waste Assay by Using Fast and Thermal Neutron Interrogation," Proc. Int. Conf. Nuclear Waste, Safewaste-2000, Montpellier, France, October 2-4 (2000) 420-429.

296 Waken, M. J. and Brownridge, M., "Enhancements in the Thorp Reprocessing Plant," Proc. ATALANTE 2004, Nîmes, France, June 21-24 (2004) O1-01.

297 Golovkov, V. M., Chakhlov, V. L., and Shtein, M. M., "Detection of Fissioning Materials Using a Neutron Source Based on a Compact Betatron," Atomic Energy 96 (2004) 127-132.

298 Rooney, B. D., et al., "Active Neutron Interrogation Package Monitor," IEEE Nucl. Sci. Symp. Conf. Record, Vol. 2, Toronto, Canada, June 7-11 (1998) 1027-1030.

299 Melton, S. G., Estep, R. J., and Paterson, E. H., "Calibration of the Crated Waste Assay Monitor (CWAM) for the Low-Level Waste Measurements for the Y-12 Plant," Report LAUR-00-2468, Los Alamos National Laboratory, Los Alamos, N.M. (2000).

300 Raoux, A.-C., "Transuranic Waste Assay by Neutron Interrogation and Online Prompt and Delayed Neutron Measurement," Nucl. Inst. Meth. Phys. Res. B 207 (2003) 186-194.

301 Normand, S., et al., "Boron Loaded Plastic Scintillator Applied to Active Neutron Measurements of Waste Drums," IEEE Nucl. Sci. Symp. Conf. Rec., Vol. 2, Rome, Italy, October 16-22 (2004) 787-789.

302 Hage, W., "Nondestructive Fissile Material Assay by Induced Fission Neutron Correlation," Nucl. Inst. Meth. Phys. Res. A 551 (2005) 396-419.

303 Kunz, W. E., et al., "A 1-mg-Sensitivity Fissile Assay System," Proc. 3rd Annual ESARDA Symp., on Safeguards and Nuclear Materials Management, Karlsruhe, West Germany, May 68 (1981). 
304 Hausladen, P. A., "Portable Fast-Neutron Radiography with the Nuclear Materials Identification System for Fissile Material Transfers," Nucl. Inst. meth. Phys. Res. B 261 (2007) 387-390.

305 Maksyutenko, B. P., "Optimum Neutron Spectrum for Activating Fuel Pins in DelayedNeutron Monitoring," Atomic Energy 48 (1980) 344-345.

306 Tobin, S. J., et al., "Determination of Plutonium Content in Spent Fuel with NDA-Why an Integrated Approach?," Report LA-UR-08-03763, Los Alamos National Laboratory, Los Alamos, N.M. (2008).

307 McConchie, S., et al., "Pulsed D-D Neutron Generator Measurements of HEU Oxide Fuel Pins," AIP Conf. Proc. 1099 (2009) 643-646.

308 Chichester, D. L. and Seabury, E. H., "Active Interrogation Using Electronic Neutron Generators for Nuclear Safeguards Applications," AIP Conf. Proc. 1099 (2009) 851-856.

309 Krinninger, H., Wiesner, S., and Faber, C., "Pulsed Neutron Method for Non-Destructive and Simultaneous Determination of the ${ }^{235} \mathrm{U}$ and ${ }^{239} \mathrm{Pu}$ Contents of Irradiated and Non-Irradiated Reactor Fuel Elements," Nucl. Inst. Meth. 73 (1969) 13-33.

310 Krinninger, H., Ruppert, E., and Siefkes, H., "Operational Experience with the Automatic Lead-Spectrometer Facility for Nuclear Safeguards," Nucl. Inst. Meth. 117 (1974) 61-84.

311 Vanterpool, E. C., et al., "Fuel Assembly Assay by Neutron Interrogation in a Lead SlowingDown-Time Spectrometer," Nucl. Sci. Eng. 110 (1992) 186-194.

312 Lee, Y.D., et al., Design of a Spent Fuel Assay Device Using a Lead Spectrometer," Nucl. Sci. Eng. 131 (1999) 45-61.

313 Lee, Y.D., et al., "Neutron Tomographic Fissile Assay in Spent Fuel Using the Lead Slowing Down Time Spectrometer," Nucl. Inst. Meth. Phys. Res. A 459 (2001) 365-376.

314 Gavron, A. Smith, L. E., and Ressler, J. J., "Analysis of Spent Fuel Assemblies Using a Lead Slowing Down Spectrometer," Nucl. Inst. Meth. Phys. Res. A 602 (2009) 581-587.

315 Fetter, S., et al., "Detecting Nuclear Warheads," Sci. Global Security 1 (1990) 225-302.

316 Koonin, S., et al., "Concealed Nuclear Weapons," Report JSR-03-130, JASON Study Group, The MITRE Corporation, McLean, Va. (2003).

317 Moss, C. E., et al., "Comparison of Active Interrogation Techniques," IEEE Trans. Nucl. Sci. 53 (2006) 2242-2246.

318 Detecting Nuclear and Radiological Materials, Royal Society Policy Document 07/08, The Royal Society, London, U.K. (2008).

319 American National Standard Minimum Performance Criteria for Actvie Interrogation Systems Used for Homeland Security, Standard ANSI N42.41-2007, IEEE, New York, N.Y. (2008).

320 Kerr, P., et al., "Active Detection of Small Quantities of Shielded Highly-Enriched Uranium Using Low-Dose 60-keV Neutron Interrogation," Nucl. Inst. Meth. Phys. Res. B 261 (2007) 347-350.

321 Bogolubov, Y. P., et al., "Method and System Based on Pulsed Neutron Generator for Fissile Material Detection in Luggage," Nucl. Inst. Meth. Phys. Res. B 213 (2004) 439-444.

322 Jordan, K. A., "Detection of Special Nuclear Material in Hydrogenous Cargo Using Differential Die-Away Analysis," Ph.D. Thesis, University of California, Berkley, Berkeley, Calif. (2006).

323 Chichester, D. L. and Seabury, E. H., "Using Electronic Neutron Generators in Active Interrogation to Detect Shielded Fissionable Material," IEEE Trans. Nucl. Sci. 56 (2009) 441447.

324 Jordan, K. A. and Gozani, T., "Detection of ${ }^{235} \mathrm{U}$ in Hydrogenous Cargo with Differential DieAway Analysis and Optimized Neutron Detectors," Nucl. Inst. meth. Phys. Res. A 579 (2007) 388-390.

325 Jordan, K. A., et al., "Improving Differential Die-Away Analysis Via the use of Neutron Poisons in Detectors," Nucl. Inst. Meth. Phys. Res. A 579 (2007) 404-406. 
326 Jordan, K. A., Vujic, J., and Gozani, T., "Remote Thermal Neutron Die-Away Measurements to Improve Differential Die-Away Analysis," Nucl. Inst. Meth. Phys. Res. A 579 (2007) 407409.

327 Jordan, K. A. and Gozani, T., "Pulsed Neutron Differential Die Away Analysis for Detection of Nuclear Materials," Nucl. Inst. Meth. Phys. Res. B 261 (2007) 365-368.

328 Jordan, K. A., Gozani, T., and Vujic, J., "Differential Die-Away Analysis System Response Modeling and Detector Design," Nucl. Inst. Meth. Phys. Res. A 589 (2008) 436-444.

329 Chichester, D. L. and Seabury, E. H., "Active Neutron Interrogation to Detect Shield Fissionable Material," Paper SM/EN-0, Proc. Int. Topical Meeting on Nuclear Research Applications and Utilization of Accelerators, International Atomic Energy Agency, Vienna, Austria, May 4-9 (2009).

330 Rosenstock, W. and Köble, T., "Detection of Shielded or Hidden Nuclear Material," Proc. Joint ESARDA/INMM Workshop on Science and Modern Technology for Safeguards, Arona, Italy, October 26-30 (1996) 29-33.

331 Rosenstock, W., Köble, T., and Hilger, P., "Measurement of the Time Structure of Delayed Neutrons after Induced Fission in Special Nuclear Material," Proc. 21st ESARDA Symp. on Safeguards and Nuclear Material Management, Seville, Spain, May 4-6 (1999) 321-325.

332 Moss, C. E., et al., "Neutron Detectors for Active Interrogation of Highly Enriched Uranium," IEEE Trans. Nucl. Sci. 51 (2004) 1677-1681.

333 Moss, C. E., et al., "Portable Active Interrogation System," Nucl. Inst. Meth. Phys. Res. B 241 (2005) 793-797.

334 Myers, W., Goulding, C. A., and Hollas, C. L., "Determination of the 235U Enrichment of Bulk Uranium Samples Using Delayed Neutrons," Report LA-UR-06-3984, Los Alamos National Laboratory, Los Alamos, N.M. (2006).

335 Keepin, G. R., Wimett, T. F., and Zeigler, R. K., "Delayed Neutrons from Fissionable Isotopes of Uranium, Plutonium, and Thorium," Phys. Rev. 107 (1957) 1044-1049.

336 Norman, E. B., et al., "Signatures of Fissile Materials: High-Energy $\gamma$ Rays Following Fission," Nucl. Inst. Meth. Phys. Res. A 51 (2004) 608-610.

337 Slaughter, D. R., et al., "The "Nuclear Car Wash": A Scanner to Detect Illicit Special Nuclear Material in Cargo Containers," IEEE Sensors J. 5 (2005) 560-564.

338 Slaughter, D. R., et al., "The Nuclear Car Wash: A System to Detect Nuclear Weapons in Commercial Cargo Shipments," Nucl. Inst. meth. Phys. Res. A 579 (2007) 349-352.

339 Hall, J. M., et al., "The Nuclear Car Wash: Neutron Interrogation of Cargo Containers to Detect Hidden SNM," Nucl. Inst. Meth. Phys. Res. B 261 (2007) 337-340.

340 Dougan, A., et al., "Screening of Maritime Containers to Intercept Weapons of Mass Destruction," Report UCRL-TR-202775, Lawrence Livermore National Laboratory, Livermore, Calif. (2004).

341 Hollas, C. L., Goulding, C., and Myers, B., "Non Destructive Analysis of Shielded Highly Enriched Uranium," Report IAEA-SM-367/17/06, International Atomic Energy Agency, Vienna, Austria (2001).

342 Mihalczo, J. T., et al., "NMIS Plus Gamma Spectroscopy for Attributes of HEU, PU, and HE Detection," Nucl. Inst. Meth. Phys. Res B 213 (2004) 378-384.

343 Mullens, J. A., et al., "Fast Coincidence Counting with Active Inspection Systems," Nucl. Inst. Meth. Phys. Res. B 241 (2005) 804-809.

344 Verbeke, J. M., et al., "Neutron Correlations in Special Nuclear Materials, Experiments and Simulations," Report UCRL-PROC-231582, Lawrence Livermore National Laboratory, Livermore, Calif. (2007).

345 Ellis, D. V., et al., "Adapting Wireline Logging Tools for Environmental Logging Applications," Trans. SPWLA 36th Annual Logging Symposium, Paris, France, June 26-29 (1995). 
346 Shapiro, J. B., James, W. D., and Schweikert, E. A., "A Preliminary Screening Technique for Selected Metals at Waste Sites," J. Radioanal. Nucl. Chem. 192 (1995) 275-280.

347 Shultis, J. K., et al., "Determining Soil Contamination Profiles from Intensities of CaptureGamma Rays Using Above-Surface Neutron Sources," App. Rad. Iso. 54 (2001) 565-583.

348 Dulloo, A. R., et al., "Detection Limits of a Laboratory Pulsed Gamma Neutron Activation Analysis System for the Nondestructive Assay of Mercury, Cadmium, and Lead," Nucl. Tech. 123 (1998) 103-112.

349 Gehrke, R. J. and Streier, G. G., "Performance test Results of Noninvasive Characterization of Resource Conservation and Recovery Act Surrogate Waste by Prompt Gamma Neutron Activation Analysis," Report INEL/EXT-97-00141, Idaho National Engineering and Environmental Laboratory, Idaho Falls, Id. (1997).

350 Dulloo, A. R., et al., "Pulsed Gamma Neutron Activation Analysis (PGNAA) System for the Assay of RCRA Metals in Mixed Waste," Proc. 1999 Industry Partnerships to Deploy Environmental Technology Conference, Morgantown, W. Va., October 12-14 (1999).

351 Dulloo, A. R., et al., "Neutron Fluence Rate Measurements in a PGNAA 208-Liter Drum Assay System Using Silicon Carbide Detectors," Nucl. Inst. Meth. Phys. Res. B 213 (2004) 400-405.

352 Wielopolski, L., et al., "Soil Carbon Measurements Using Inelastic Neutron Scattering," IEEE Trans. Nucl. Sci. 47 (2000) 914-917.

353 Wielopolski, L., et al., "Non-Destructive Soil Carbon Analyzer (ND-SCA) Progress Report," Report BNL-72200-2004, Brookhaven National Laboratory, Upton, N.Y. (2004).

354 Mitra, S., et al., "Concurrent Measurement of Individual Gamma-Ray Spectra During and Between Fast Neutron Pulses," IEEE Trans. Nucl. Sci. 54 (2007) 192-196.

355 Roquemore, A. L., et al., "Performance of a 14-MeV Neutron Generator as an In Situ Calibration Source for TFTR," Report LA-UR-93-4234, Los Alamos National Laboratory, Los Alamos, N.M. (1993).

356 Blaufuss, E., "16 $\mathrm{N}$ as a Calibration Source for Super-Kamiokande," Nucl. Inst. Meth. Phys. Res. A 458 (2001) 638-649.

357 Tagg, N. J., "The 8Li Calibration Source for the Sudbury Neutrino Observatory," Nucl. Inst. Meth. Phys. Res. A 489 (2002) 178-188.

358 Bauer, J. M., "Absolute Energy Calibration with the Neutron-Activated Liquid-Source System at BaBar's CsI(Tl) Calorimeter," Report SLAC-PUB-10289, Stanford Linear Accelerator Center, Stanford, Calif. (2003).

359 Kliment, V. and Tölgyessy, J., "Continuous Neutron Activation Analysis," J. Radioanal. Nucl. Chem. 10 (1972) 273-297.

360 Perez-Griffo, M. L., Block, R. C., and Lahey, R. T., "Measurement of Flow in Large Pipes by Pulsed Neutron Activation Analysis Methods," Nucl. Sci. Eng. 82 (1982) 19-33.

361 Caldwell, R. L., et al., "Combination Neutron Experiment for Remote Analysis," Science 152 (1966) 457-465.

362 Johnson, R. G., Evans, L. G., and Trombka, J. I., "Neutron-Gamma Techniques for Planetary Exploration," IEEE Trans. Nucl. Sci. 26 (1979) 1574-1578.

363 Bradley, J. G., et al., "A Neutron Activation Gamma Ray Spectrometer for Planetary Surface Analysis," Acta Astonautica 35 Suppl. (1995) 109-118.

364 Schweitzer, J. S., "Neutron-Induced Gamma-Ray Measurements on Soft Landers," Nucl. Inst. Meth. Phys. Res. A 422 (1999) 595-599.

365 Akkurt, H., et al., "Pulsed Neutron Generator System for Astrobiological and Geochemical Exploration of Planetary Bodies," Nucl. Inst. Meth. Phys. Res. B 241 (2005) 232-237.

366 Busch, M. W. and Aharonson, O., "Measuring Subsurface Water Distribution Using the Dynamic Albedo of Neutrons Instrument on Mars Science Laboratory," Nucl. Inst. meth. Phys. Res. A 592 (2008) 393-399. 
367 Litvak, M. L., et al., "The Dynamic Albedo of Neutrons (DAN) Experiment for NASA's 2009 Mars Science Laboratory," Astrobiology 8 (2008) 605-612.

368 "Radiation Source Use and Replacement," National Academies Press, Washington, D.C. (2008).

369 Reijonen, J., Leung, K. N., and Jones, G., "RF Ion Source Development for Neutron Generation and for Material Modification," rev. Sci. Inst. 73 (2002) 934-936.

370 Kim, I. J. and Choi, H. D., "Development of D-D Neutron Generator," Nucl. Inst. Meth. Phys. Res. B 241 (2005) 917-920.

371 Schwoebel, P. R., "Field Desorption Ion Source for Neutron Generators," App. Phys. Lett. 87 (2005) 054104.

372 Chichester, D. L., et al., "Development of a Field Desorption Ion Source for Neutron Generator Applications," Nucl. Inst. Meth. Phys. Res. B 261 (2007) 835-838.

373 Solano, I., et al., Field Desorption Ion Source Development for Neutron Generators," Nucl. Inst. Meth. Phys. Res. A 587 (2008) 76-81.

374 Hausladen, P. A., Neal, J. S., and Mihalczo, J. T., "An Alpha Particle Detector for a Portable neutron Generator for the Nuclear materials Identification System (NMIS), "Nucl. Inst. Meth. Res. Phys. Res. B 241 (2005) 835-838.

375 Neal, J. S., et al., "Evaluation of Melt-Grown, ZnO Single Crystals for Use As Alpha Particle Detectors," IEEE Trans. Nucl. Sci. 55 (2008) 1397-1403.

376 Naranjo, B., Gimzewski, J. K., and Putterman, S., "Observation of Nuclear Fusion Driven by a Pyroelectric Crystal," Nature 434 (2005) 1115-1117.

377 Geuther, J. A. and Danon, Y., "Electron and Positive Ion Acceleration with Pyroelectric Crystals,” J. App. Phys. 97 (2005) 074109. 